\title{
Invariants of Three-Manifolds, Unitary Representations of the Mapping Class Group, and Numerical Calculations
}

\author{
Michael Karowski, Robert Schrader, and Elmar Vogt
}

\section{CONTENTS}

1. Introduction

2. Invariants of Coloured Graphs

3. Finite-Dimensional Unitary Representations of the Mapping Class Group

4. Application to the Partition Function of Arbitrary Manifolds

5. Numerical Results

Appendix A. Proof of an Orthogonality Relation for the

Canonical Graph of Handlebodies

Appendix B. Proof of Some Equations of Section 3

Appendix C. Chern-Simons Invariants for Flat Connections

Appendix D. Vanishing Invariants

Final Note

References

\begin{abstract}
Based on previous results of the two first authors, it is shown that the combinatorial construction of invariants of compact, closed three-manifolds by Turaev and Viro as state sums in terms of quantum $6 \mathrm{j}$-symbols for $\mathrm{SL}_{q}(2 ; \mathbb{C})$ at roots of unity leads to the unitary representation of the mapping class group found by Moore and Seiberg. Via a Heegaard decomposition this invariant may therefore be written as the absolute square of a certain matrix element of a suitable group element in this representation. For an arbitrary Dehn surgery on a figure-eight knot we provide an explicit form for this matrix element involving just one $6 \mathrm{j}$-symbol. This expression is analyzed numerically and compared with the conjectured large $k=r-2$ asymptotics of the Chern-Simons-Witten state sum [Witten 1989], whose absolute square is the Turaev-Viro state sum. In particular we find numerical agreement concerning the values of the Chern-Simons invariants for the flat $\mathrm{SU}(2)$-connections as predicted by the asymptotic expansion of the state sum with analytical results found by Kirk and Klassen [1990].
\end{abstract}

\section{INTRODUCTION}

The advent of topological quantum field theories has stimulated the analysis of compact three-manifolds and knot theory. Thus Witten [1989] (see also [Fröhlich and King 1989]) suggested a functional integral approach using the Chern-Simons action (with a coupling constant $k \in \mathbb{Z}$, called the level) to obtain Jones' polynomial knot invariants [Jones $1985 ; 1987$ as expectation values of Wilson loops. In particular the partition function $\tau(M, k)$ itself should give rise to new invariants of three-manifolds $M$. The idea of Witten was made rigorous by Reshetikhin and Turaev [1990; 1991] without 
recourse to the as yet formal functional integral approach by using the theory of braided ribbon graphs associated to quantum groups, in particular for the quantum group $\mathrm{SL}_{q}(2, \mathbb{C})$ at roots of unity $q=\exp \pi i / r$, with $r=k+2$. The correct normalization of the Reshetikhin-Turaev invariants was provided by Kirby and Melvin [1991], who also analyzed these invariants for small $r$.

Another approach, at first sight seemingly unrelated, was advocated in [Turaev and Viro 1992], and investigated further by Turaev [1991a; 1991b; 1991c; 1992; 1993]. It uses quantum $6 j$-symbols, in particular those of $\mathrm{SL}_{q}(2, \mathbb{C})$ at roots of unity. This approach was generalized by Durhuus, Jakobsen, and Nest [Durhuus et al. 1993; Durhuus 1993] to a large class of algebras (replacing $\mathrm{SL}_{q}(2, \mathbb{C})$ ) with associated $6 j$-symbols. Using a triangulation of a closed three-manifold $M$, these authors define an invariant, called a state sum in analogy to partition functions in statistical mechanics, and then show the independence of the particular triangulation, thus giving rise to an invariant $Z(M, r)$. As it turned out, for closed, oriented compact threemanifolds - the case we shall be dealing with exclusively in this article-the Turaev-Viro invariant and the Chern-Simons-Witten invariant are related as follows [Turaev 1991c; Walker 1991]:

$$
Z(M, r=k+2)=\left|Z_{\mathrm{CS}}(M, k)\right|^{2}
$$

The construction in [Turaev and Viro 1992] was extended in [Karowski et al. 1992] to the case where $M$ is not necessarily closed. Furthermore a direct proof was given in [Karowski and Schrader 1993] exhibiting the Turaev-Viro theory as a topological quantum field theory with a Hilbert space structure given in terms of a suitably formulated reflection positivity property [Osterwalder and Schrader 1973; 1975]. See [Beliakova and Durhuus 1995] for an extension of these results to quantum group versions of arbitrary semisimple Lie groups and an alternative proof of $(1-1)$.

Using the representations of $\mathrm{SL}(2, \mathbb{Z})$, the mapping class group for the torus, given in [Gepner and
Witten 1986], Freed and Gompf [1991] and Jeffrey [1992] have analyzed the Chern-Simons-Witten invariants for lens spaces and torus bundles over $S^{1}$. In fact, each of these invariants is then just given as a certain matrix element of a suitable mapping class group element in this representation which is obtained from the appropriate Heegaard decomposition. It also involves the use of the canonical framing given by Atiyah [1990].

One aim of this article is to show in Section 3 that the Turaev-Viro approach for $\mathrm{SL}_{q}(2, \mathbb{C})$ and arbitrary $q=\exp (i \pi / r)$ leads to a unitary representation of the mapping class group for Riemann surfaces of arbitrary genus. In fact, these representations parametrized by $r$ are exactly those found by Moore and Seiberg [1989] in the context of conformal quantum field theory. Via a suitable Heegaard decomposition, the Turaev-Viro invariant $Z(M, r)$ may be written in accordance with relation (1-1) as the absolute square of a suitable matrix element of a mapping class group element in the given representation. Our discussion so far does not permit the conclusion that the matrix element itself defines an invariant. This is related to the fact that the Turaev-Viro invariant is frame independent. Our result confirms preliminary findings of Kohno [1992; 1994], who started the opposite way, by first defining representations of the mapping class group and then showing that certain matrix elements define invariants.

For the reader's convenience, Section 2 briefly recapitulates the main concepts and results found in [Karowski and Schrader 1993]. In particular we will use certain bases in the topological quantum field theory associated to the Turaev-Viro approach. These bases are given in terms of invariants of coloured graphs on the boundary of an arbitrary oriented, compact three-manifold. The mapping class group representations considered in Section 3 are then defined in a natural way as linear transformations on this set of invariants of graphs. In Section 4 we shall give a more detailed description for the case when $M$ is obtained via an $n / m$ Dehn surgery on a figure-eight knot. In that case 
$Z(M, r)$ is given as the absolute square of an expression which involves a 3-fold sum over colours and with only one $6 j$-symbol. The resulting expression for $Z(M, r)$ cannot be simplified further in an analytic way. In Section 5, however, and stimulated by the computer calculations on lens spaces carried out by Freed and Gompf [1991], we present results from a numerical analysis for manifolds $M$ obtained in this way, which include some hyperbolic manifolds. In particular, we use our results to calculate Chern-Simons invariants of associated flat SU(2)-bundles over these manifolds as predicted by the conjecture on the asymptotic behaviour as $k \rightarrow \infty$ of the Chern-Simons-Witten state sum (see [Witten 1989; Freed and Gompf 1991; Rozansky 1995], for example), and compare them with the analytic results of Kirk and Klassen [1990]. The latter authors present tables for five Dehn surgeries on the figure-eight knot, but it is easy to extend their calculations of Chern-Simons invariants to any Dehn surgery. In all cases considered the calculations based on their method and our numerical calculation, which is based on a fundamentally different approach, give identical results (up to precision bounds) concerning the values of the Chern-Simons actions for flat bundles. Also we confirm the different asymptotic behaviour of the contributions to the state sum resulting from the irreducible and the reducible flat connections, as predicted by stationary phase methods applied to the formal functional integral. The coefficients of this asymptotic expansion are related to the Reidemeister torsion and the spectral flow of the flat bundle. In principle our numerical results also permit the evaluation of these coefficients. This could be another check of the validity of this asymptotic expansion which has not been performed so far. We note that in the case of lens spaces, Jeffrey [1992] has performed an analytic calculation of the Chern-Simons action based on Gauss's reciprocity theorem and found agreement with the well known analytic results on the flat SU(2)-bundles. Ohtsuki [1995] and Murakami [1995] have used the large $k$ expansion to obtain finite type invariants of integral homology spheres. This was extended by Rozansky [1997], Lawrence [1995] and Ohtsuki [1996] to rational homology spheres.

In the present case the relevance of the TuraevViro invariants of hyperbolic spaces for the associated fundamental groups (contained in $\mathrm{SL}(2, \mathbb{C})$ ) and the associated number theory remains an open question.

Preliminary versions of the results given here were presented at various conferences, such as the Schrödinger workshop in Vienna (1993), the AMSDMV meeting in Heidelberg (1993), and the AMSIMU meeting in Jerusalem (1995).

\section{INVARIANTS OF COLOURED GRAPHS}

In this section we give a brief review of the construction of coloured graphs in the context of the Turaev-Viro approach as given in [Karowski and Schrader 1993]. First we recall the construction of invariants of graphs on two-manifolds as boundaries of three-manifolds. Let $|G|$ be the topological space associated to a one-dimensional simplicial complex $G$ (see [Spanier 1966, pp. 108-114], for example). By assumption on $G$ every vertex $\sigma^{0} \in G$ is contained in the boundary of $n=n\left(\sigma^{0}\right)$ one-simplexes in $\mathrm{G}$ with $2 \leq n \leq 4$ and we will call $\sigma^{0}$ an $n$-vertex. The notion of being an n-vertex with $n \geq 3$ is independent of the particular triangulation $G$ of $|G|$. Every 4-vertex $\sigma^{0}$ is given an additional structure by pairing the four onesimplexes meeting in $\sigma^{0}$ into two unordered pairs. The two one-simplexes in one pair are said to be opposite to each other. In addition one of the pairs is given the name "above" and the other pair the name "below" and is depicted in Figure 1. By the preceding remark this additional structure is independent of the particular triangulation $G$ of $|G|$.

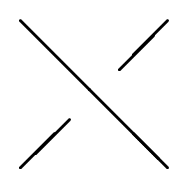

FIGURE 1. The additional structure at a 4 -vertex. 
By abuse of notation we continue to denote by $|G|$ this space equipped with this additional structure.

Let $\underline{x}: \sigma^{1} \mapsto x\left(\sigma^{1}\right)$ be a map from the set of nonoriented one-simplexes in $\mathrm{G}$ into the set of "colours" $\mathcal{J}=\left\{0, \frac{1}{2}, 1, \frac{3}{2}, \ldots, \frac{r}{2}-1\right\}$ with the following properties.

(1) If two one-simplexes $\sigma_{1}^{1}$ and $\sigma_{2}^{1}$ join at a 2 -vertex, then $x\left(\sigma_{1}^{1}\right)=x\left(\sigma_{2}^{1}\right)$.

(2) If two one-simplexes $\sigma_{1}^{1}$ and $\sigma_{2}^{1}$ are opposite to each other at a 4 -vertex, then $x\left(\sigma_{1}^{1}\right)=x\left(\sigma_{2}^{1}\right)$ (compare Figure 1).

If $\operatorname{sd} G$ is a subdivision of $\mathrm{G}$, then such a map $\underline{x}$ induces a map $\operatorname{sd} \underline{x}$ on $\operatorname{sd} G$ with similar properties by setting $\operatorname{sd} x\left(\sigma^{1^{\prime}}\right)=x\left(\sigma^{1}\right)$ whenever $\left|\sigma^{1^{\prime}}\right| \subset\left|\sigma^{1}\right|$, for $\sigma^{1^{\prime}} \in \operatorname{sd} G, \sigma^{1} \in G$. We say that a map $\underline{x}$ on $G$ and a map $\underline{x}^{\prime}$ on $G^{\prime}$ with $|G|=\left|G^{\prime}\right|$ are equivalent if they induce the same map on a common subdivision. The equivalence class is called a coloured graph, and is denoted by $|G|_{\underline{x}}$. Any set $L$ in $|G|$ homeomorphic to an interval and not containing any $n$-vertex, for $n \geq 3$, will be called a line. For given $|G|_{\underline{x}}$, by condition (1) we may associate to each line a colour $x=x(L)$. By definition, a coloured graph on a two-manifold $\Sigma$ is a pair $\left(|G|_{x}, \varphi\right)$, where $\varphi$ is a homeomorphism of $|G|$ into $\Sigma$ with the following additional property. Near the image $\varphi\left(\sigma^{0}\right)$ of a 4 -vertex $\sigma^{0}$, the images of the two open opposite one-simplexes $\in G$ in one pair are separated by the images of the closed one simplex $G$ in the other pair for any choice of a triangulation $G$ of $|G|$ (compare Figure 1). Two coloured graphs $\left(|G|_{\underline{x}}, \varphi\right)$ and $\left(|G|_{\underline{x}}, \varphi^{\prime}\right)$ on $\Sigma$ are called isotopic if there is a homotopy $\varphi_{t}(0 \leq t \leq 1)$ between the maps $\varphi$ and $\varphi^{\prime}$ such that $\left(|G|_{\underline{x}}, \varphi_{t}\right)$ are coloured graphs on $\Sigma$ for all $0 \leq t \leq 1$.

In [Karowski and Schrader 1993] we gave a generalization of the state sum $Z(M, r)$ of Turaev and Viro for the case $\mathrm{SL}_{q}(2, \mathbb{C})$, for $q=\exp i \pi / r$, assigning a complex number $Z\left(M,|G|_{\underline{x}}, \varphi\right)$ (depending on $r)$ to each triple $\left(M,|G|_{\underline{x}}, \varphi\right)$, where $M$ is an oriented, compact three-manifold and $\left(|G|_{\underline{x}}, \varphi\right)$ is a coloured graph on $\partial M$. Moreover, $Z\left(M,|G|_{\underline{x}}, \varphi\right)$ defined as a state sum is a homotopy invariant of the pair $\left(|G|_{\underline{x}}, \varphi\right)$. This understood, and by identifying $|G|$ with its image in $\partial M$ under $\varphi$, the state sum above is also simply written as $Z\left(M,|G|_{\underline{x}}\right)$.

Example 2.1. Some examples of Viro-Turaev invariants of three-manifolds that will be used in the sequel are

$$
\begin{aligned}
Z\left(D^{3}\right) & =1, \\
Z\left(S^{3}\right) & =w^{-2}, \\
Z\left(S^{2} \times S^{1}\right) & =1, \\
Z\left(S^{1} \times S^{1} \times S^{1}\right) & =(r-1)^{2}, \\
Z\left(M \backslash D^{3}\right) & =w^{2} Z(M),
\end{aligned}
$$

where $w$ will be defined in Equation (2-9). For details, see, for example, [Karowski et al. 1992; Karowski and Schrader 1993].

The next lemma gives factorization formulas for the invariants of three-manifolds equipped with coloured graphs, derived in [Karowski et al. 1992; Karowski and Schrader 1993] (see also [Karowski 1992]).

Lemma 2.2. (a) If a three-manifold consists of two disconnected parts $M \cap M^{\prime}=\varnothing$ such that $|G|_{\underline{a}} \subset$ $\partial M$ and $\left|G^{\prime}\right|_{\underline{a}^{\prime}} \subset \partial M^{\prime}$, then

$$
Z\left(M \cup M^{\prime},|G|_{\underline{a}} \cup|G|_{\underline{a}^{\prime}}^{\prime}\right)=Z\left(M,|G|_{\underline{a}}\right) Z\left(M^{\prime},|G|_{\underline{a}^{\prime}}^{\prime}\right) .
$$

(b) If $|G|_{\underline{a}}$ is planar, that is, if $|G|$ is contained in an open set of $\partial M$ homeomorphic to a disc, then $Z\left(M,|G|_{\underline{a}}\right)$ factorizes:

$$
Z\left(M,|G|_{\underline{a}}\right)=Z(M) Z\left(|G|_{\underline{a}}\right)
$$

[Karowski and Schrader 1993, Theorem 4.2]. In this equality $Z\left(|G|_{\underline{a}}\right)$ is independent of $M$ and is called the invariant of the planar graph $|G|_{\underline{a}}$. Since $Z\left(D^{3}\right)=1$ for the unit ball $D^{3}$ in $\mathbb{R}^{3}$ with boundary $\cong S^{2}$ (see Example 2.1), we have

$$
Z\left(|G|_{\underline{a}}\right)=Z\left(D^{3},|G|_{\underline{a}}\right) .
$$

This expression is not only homotopy invariant but also invariant under Reidemeister moves. 
(c) If a graph consists of disconnected planar parts $\left|G_{1}\right|_{\underline{a}} \cap\left|G_{2}\right|_{\underline{b}}=\varnothing$ we have

$$
Z\left(\left|G_{1}\right|_{\underline{a}} \cup\left|G_{2}\right|_{\underline{b}}\right)=Z\left(\mid G_{1} \underline{\underline{a}}_{\underline{a}}\right) Z\left(\left|G_{2}\right|_{\underline{b}}\right) .
$$

We also recall some examples of invariants of planar graphs that will be used later. The invariant of the circle with colour $i \in \mathcal{J}$, namely

$$
Z(\bigcirc i)=w_{i}^{2}=(-1)^{2 i} \frac{q^{2 i+1}-q^{-2 i-1}}{q-q^{-1}},
$$

yields $(-1)^{2 i}$ times the $q$-dimension of the irreducible representation of $\mathrm{SL}_{q}(2, \mathbb{C})$ associated to the colour $i$. The fusion matrix $N_{j k}^{i}$, for $i, j, k \in \mathcal{J}$, is obtained by

$$
Z(i \bigcirc j)=N_{j k}^{i}=\left\{\begin{array}{c}
1 \quad \begin{array}{c}
\text { if } k \leq i+j, j \leq i+k \\
i \leq k+j, \text { and } \\
r-2 \geq i+j+k \in \mathbb{Z} \\
0 \quad \text { otherwise }
\end{array}
\end{array}\right.
$$

with $N_{j k}^{0}=\delta_{j k}$. The invariant

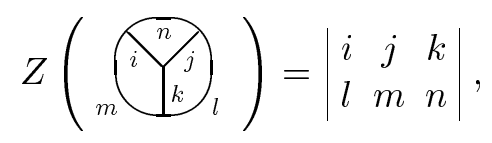

with $i, \ldots, n \in \mathcal{J}$, is equal to the $q$-Racah-Wigner $6 j$-symbol, and

$$
Z\left({ }_{a}\left(\begin{array}{c}
d> \\
i
\end{array}\right)=\frac{q_{a} q_{b}}{q_{c} q_{d}}\left|\begin{array}{lll}
i & a & c \\
j & b & d
\end{array}\right|\right.
$$

is equal to the $R$-matrix (in the path basis) representing the 4-vertex of Figure 1. Here

$$
q_{a}=(-1)^{-a} q^{a(a+1)}
$$

for $a \in \mathcal{J}$, correcting a misprint in [Karowski and Schrader 1993, (2.11)].

Various relations of invariants of graphs [Karowski and Schrader 1993] such as completeness, orthogonality, Yang-Baxter's relation, Racah's relation, and Biedenharn-Elliot's relation (also called the Fierz transformation) are obtained from a cutting rule for graphs, given by the Wigner-Eckart relation

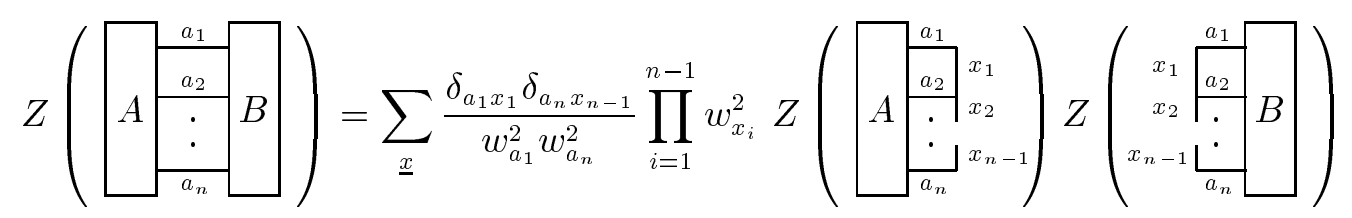

For $n=1$ the single colour $a_{1}$ has to be zero. Using this cutting rule any planar graph may be cut into pieces of the form of Equations $(2-2)-(2-5)$. Therefore:

Lemma 2.3. The invariant of any planar graph is the sum of products of invariants of the simple graphs of Equations (2-2)-(2-5); that is, it can be expressed in terms of $w_{i}^{2}, q_{i}$ and the $6 j$-symbols.

In [Karowski and Schrader 1993] the construction of $Z\left(M,|G|_{\underline{x}}\right)$ was also extended to coloured graphs in the interior of the three-manifold $M$ as follows:

Definition 2.4. Let $M$ be a compact three-manifold. A coloured graph $\mathcal{G}_{\underline{x}}$ in $M$ is a triple

$$
\left(c\left(\mathcal{G}_{\underline{x}}\right), \mathcal{T}_{\mathcal{G}_{\underline{x}}},|G|_{\underline{x}}\right)
$$

satisfying these conditions: (a) $c\left(\mathcal{G}_{\underline{x}}\right)$ is a finite one-dimensional simplicial complex embedded in $\stackrel{\circ}{M}$ and called the core of $\mathcal{G}_{\underline{x}}$.

(b) $\mathcal{T}_{\mathcal{G}_{\underline{x}}}$ is an open tubular neighborhood of $c\left(\mathcal{G}_{\underline{x}}\right)$ in $\stackrel{\circ}{M}$ with smooth boundary $\partial \mathcal{T}_{\mathcal{G}_{\underline{x}}}$.

(c) $|G|_{\underline{x}}$ is a coloured graph on $\partial \mathcal{T}_{\mathcal{G}_{\underline{x}}}$.

Given $\mathcal{G}_{\underline{x}}$, we denote by $M \backslash \mathcal{T}_{\mathcal{G}_{\underline{x}}}$ the compact oriented three-manifold obtained by deleting $\mathcal{T}_{\mathcal{G}_{\underline{x}}}$ from $M$. By construction the boundary of $M \backslash \mathcal{T}_{\mathcal{G}_{\underline{x}}}$ is the disjoint union of $\partial \mathcal{T}_{\mathcal{S}_{\underline{x}}}$ and $\partial M$. By definition, the invariant of the three-manifold $M$ equipped with a coloured graph in its interior is

$$
Z\left(M\left(\mathcal{G}_{\underline{x}}\right)\right)=Z\left(M \backslash \mathcal{T}_{\mathcal{G}_{\underline{x}}},|G|_{\underline{x}}\right) .
$$

Of course this definition may be extended to the case where there is another coloured graph $\left|G^{\prime}\right|_{\underline{x}^{\prime}}$ on $\partial M$. This notion of a coloured graph $\mathcal{G}_{\underline{x}}$ in $\bar{M}$ 
allows the introduction of what we called a meridian $m$ in [Karowski and Schrader 1993]:

Definition 2.5. Consider a subset in $\mathcal{T}_{\mathcal{G}_{\underline{x}}}$ that looks like $\stackrel{\circ}{D}^{2} \times I\left(D^{2}\right.$ the unit disc in $\mathbb{R}^{2}, I=[0,1]$ the unit interval), and such that its intersection with $\partial \mathcal{T}_{\mathcal{G}_{\underline{x}}}$ has the form $\left(S^{1}=\partial D^{2}\right) \times I$. A meridian $m$ is any circle of the form $S^{1} \times\{Q\}$, with $Q \in(0,1) \subset I$. Such a meridian will be equipped with a colour $y$ and combined with $|G|_{\underline{x}}$ to give a coloured graph denoted by $|G \cup m|_{\underline{x}, y}$. Given several such meridians written as $\underline{m}=m_{1} \cup \cdots \cup m_{n}$ and equipped with colours $\underline{y}=\left(y_{1}, \ldots, y_{n}\right)$, by definition the invariant of the three-manifold $M$ containing the graph

$$
\mathcal{G}_{\underline{\underline{x}}}^{\underline{m}}=\left(c\left(\mathcal{G}_{\underline{x}}\right), \mathcal{T}_{\mathcal{G}_{\underline{x}}},|G \cup \underline{m}|_{\underline{x}, \underline{y}}\right)
$$

with meridians $\underline{m}$ is

$$
Z\left(M\left(\mathcal{G}_{\underline{\underline{x}}}^{\underline{m}}\right)\right)=\sum_{\underline{y}} \prod_{i=1}^{n} \frac{w_{y_{i}}^{2}}{w^{2}} Z\left(M \backslash \mathcal{T}_{\mathcal{G}_{\underline{x}}},|G \cup \underline{m}|_{\underline{x}, \underline{y}}\right),
$$

where

$$
w^{2}=\sum_{i} w_{i}^{4}=\frac{-2 r}{\left(q-q^{-1}\right)^{2}}, \quad q=e^{i \pi / r}
$$

and $w_{i}^{2}$ is given by Equation (2-2). A line of $\mathcal{G}_{\underline{x}}$ is called oriented if it intersects a meridian once transversally. It is called left (right) handed if it undercrosses (overcrosses) the meridian in the sense of Figure 1, looking from the exterior of $\mathcal{T}_{\mathcal{G}_{\underline{x}}}$ onto the 4 -vertex.

Graphically a left-handed and a right-handed line look locally like

$$
\uparrow . \mid=m \text { and }\left|\frac{1 .}{1}\right| m \text { respectively. }
$$

We will need later the following projecting and branching properties of meridians [Karowski and Schrader 1993, Corollaries 5.5, 5.6 and 6.5].
Lemma 2.6. (i) If one of the colours $x$ in $\underline{x}$ happens to be simultaneously left- and right-handed, the invariant (2-8) vanishes unless $x=0$.

$$
\sum_{y y^{\prime}} \frac{w_{y}^{2}}{w^{2}} \frac{w_{y^{\prime}}^{2}}{w^{2}} \quad \|_{x} y_{y}^{y^{\prime}} \propto \delta_{x 0}
$$

(ii) If $m$ and $m^{\prime}$ are two meridians of the same lefthanded (say) line, one of them may be omitted:

$$
m \cup m^{\prime} \equiv m \quad \text { or } \mapsto \equiv H .
$$

(iii) If three left-handed (say) lines with meridians $m, m^{\prime}$ and $m^{\prime \prime}$ branch at a three vertex, one of the meridians may be omitted: $m \cup m^{\prime} \cup m^{\prime \prime} \equiv$ $m \cup m^{\prime}$, or

$$
\underline{T} \equiv \bar{r} .
$$

Similar relations hold for right-handed lines. The relations above have to be understood in the sense that both sides will give the same invariant in formula (2-8).

In the following results all lines will be assumed to be oriented. We write for short $Z\left(M, \mathcal{G}_{\underline{a}, \underline{\underline{b}}}\right)$ if the graph has left-handed lines with colours $\underline{a}$ and right-handed ones with colours $\underline{\bar{b}}$.

Lemma 2.7 [Karowski and Schrader 1993]. The relative braiding of a left- and a right-handed line is trivial:

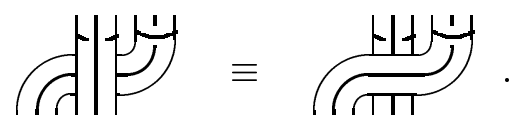

Corollary 2.8. A graph $\mathcal{G}_{\underline{a}, \underline{b}}$ in $S^{3}$ with oriented lines can be decomposed as $\overline{\mathcal{G}}_{\underline{a}} \cup \mathcal{G}_{\underline{b}}$ such that $\mathcal{G}_{\underline{a}} \subset D_{1}^{3}$, $\mathcal{G}_{\bar{b}} \subset D_{2}^{3}$ and $D_{1}^{3} \cap D_{2}^{3}=\varnothing$. Therefore the invariant factorizes

$$
Z\left(S^{3}\left(\mathcal{G}_{\underline{a}, \bar{b}}\right)\right)=w^{2} Z\left(S^{3}\left(\mathcal{G}_{\underline{a}}\right)\right) Z\left(S^{3}\left(\mathcal{G}_{\bar{b}}\right)\right) .
$$

In addition, in [Karowski and Schrader 1993] formulas were derived that express the change of the invariants of three-manifolds equipped with graphs under specific changes $M \rightarrow M^{\prime}$ of the manifolds. 
Lemma 2.9 (Cutting full cylinders). If the three-manifold $M$ contains a cylinder $D^{2} \times[0,1]$ such that $\partial D^{2} \times[0,1] \subset \partial M$ and if, for a point $P \in \partial D^{2}$, $P \times[0,1]$ is part of a line (of colour a) of a graph $G_{\underline{a}}$, then the cylinder may be removed using the formula

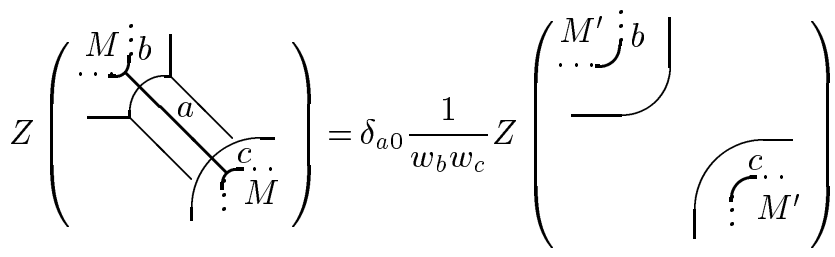

Lemma 2.10 (Introducing hollow tubes). If the threemanifold $M$ contains a cylinder $D^{2} \times[0,1]$ such that $D^{2} \times\{0,1\} \subset \partial M$, then the cylinder may be removed (that is, a tube may be introduced) using the formula

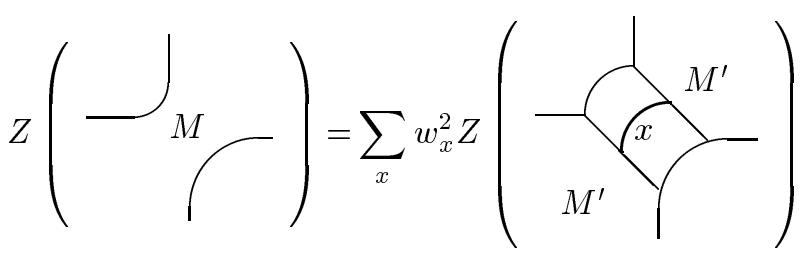

where the circle $\partial D^{2} \times\left\{\frac{1}{2}\right\}$ with colour $x$ is a meridian.

Finally we have a relation between invariants of graphs in the internal of three-manifolds and the invariants of planar graphs. For the simple case of $S^{3}$ this is given by the following theorem.

Theorem 2.11. For any graph $\mathcal{G}_{\underline{a}} \subset S^{3}$ (of only lefthanded lines) the invariant of $S^{3}\left(\mathcal{G}_{\underline{a}}\right)$ may be written in terms of the invariant of a corresponding planar graph $|G|_{\underline{a}}$ on $S^{2}$

$$
Z\left(S^{3}\left(\mathcal{G}_{\underline{a}}\right)\right)=w^{\chi\left(\mathcal{G}_{\underline{a}}\right)-2} Z\left(|G|_{\underline{a}}\right),
$$

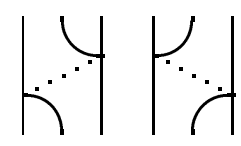

(a)

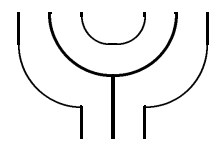

(c)

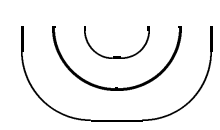

(d)

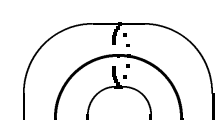

(e) where $\chi\left(\mathcal{G}_{a}\right)$ is the Euler characteristic of the graph $\mathcal{G}_{\underline{a}}$ and $|G|_{\underline{a}}$ is some projection of $\mathcal{G}_{\underline{a}} \subset S^{3}$ onto an $S^{2} \subset S^{3}$.

The exact form of the planar graph $|G|_{\underline{a}}$ follows from the construction in the proof.

Proof. We use homotopy invariance, apply iteratively the Wigner-Eckart relation (2-6), and then change the graph $\mathcal{G}_{\underline{a}} \subset S^{3}$ in such a way that its projection onto $\mathbb{R}^{2}$ may be decomposed into local pieces that look like those of Figure 2.

All meridians may be shifted by Lemma 2.6 so that they only appear at pieces of type (e). Now we use properties of the meridians [Karowski and Schrader 1993, Lemma 5.4] to change pieces (a) and (b) of Figure 2 as follows:

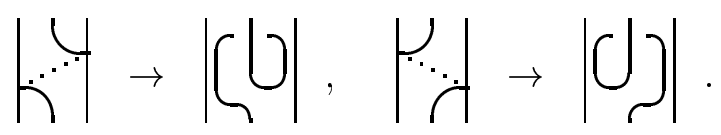

Creating a hole in $S^{3}$ such that its projection lies completely below the the projection of the graph, the invariant changes by a factor $w^{-2}$ : indeed,

$$
Z\left(S^{3}\left(\mathcal{G}_{\underline{a}}\right)\right)=w^{-2} Z\left(\left(S^{3} \backslash D^{3}\right)\left(\mathcal{G}_{\underline{a}}\right)\right)
$$

(see Example 2.1). Now we deform the hole so that in the end the original position of the graph lies completely inside the hole. During this procedure we perform step by step several changes of the graph and the manifold. At the end the tubular neighborhood has disappeared and only the hole remains with the graph $|G|_{\underline{a}}$ on its boundary $S^{2}$. If, during this procedure, we reach a piece of type

FIGURE 2. The building blocks of any graph $\mathcal{G}_{\underline{a}}$.

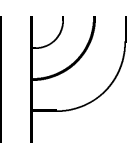

(f)

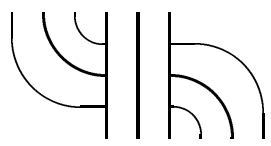

(g)

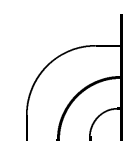


(d), we apply Lemma 2.10 to connect it with the hole:

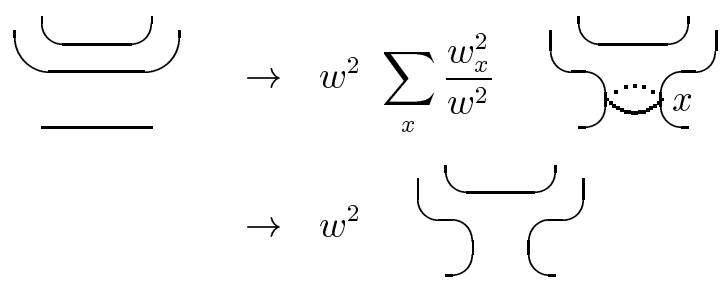

(In second step we have used Lemma 2.6 again.) Thus the invariant has changed by a factor of $w^{2}$. Passing pieces of type (a), (b), (f), and (g) the invariant will not change. In the case of (f) we use again [Karowski and Schrader 1993, Lemma 5.4] to obtain

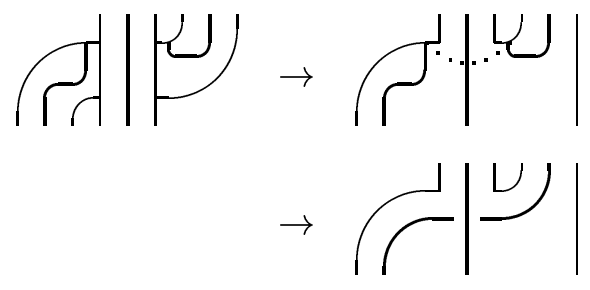

and analogously for case $(\mathrm{g})$. If we reach a piece of type (e) we apply Lemma 2.9:

$$
\begin{aligned}
\sum_{x} \frac{w_{x}^{2}}{w^{2}} & \rightarrow \sum_{x} \frac{w_{x}^{2}}{w^{2}} \delta_{x 0} \\
& \rightarrow \frac{1}{w^{2}}
\end{aligned}
$$

The invariant changes by a factor $w^{-2}$. Finally the graph $\mathcal{G}_{\underline{a}}$ has disappeared and instead we have the new planar graph $|G|_{\underline{a}}$ on the boundary $\cong S^{2}$ where each piece of $\mathcal{G}_{\underline{a}}$ as depicted in Figure 2 is replaced by the corresponding piece of $|G|_{\underline{a}}$, as depicted in Figure 3.
Since $Z\left(S^{3} \backslash D^{3}\right)=1$ and twice the number of pieces of type (e) minus twice the number of pieces of type (d) is the Euler characteristic of $\mathcal{G}_{\underline{a}}$, the claim follows.

For a graph $\mathcal{G}_{\underline{\underline{b}}} \subset S^{3}$ with only right-handed lines the result is quite similar. The only difference is that for the resulting planar graph $|G|_{\underline{b}} \subset S^{2}$, in cases (a), (b), (f), and (g), overcrossings and undercrossings are exchanged. This means that the invariant has the complex conjugate value compared to the corresponding left-handed case.

We now define basic graphs in the interior of handle bodies. We use here a slight modification of [Karowski and Schrader 1993, Definition 7.5], since this turns out to be more convenient for our construction of the representation of the mapping class group.

Consider the handlebody (gefillte pretzel) $M_{\Sigma}$ associated to a Riemann surface $\Sigma$ of genus $g \geq 2$. Let $\left(\alpha_{1}, \ldots, \alpha_{g}, \beta_{1}, \ldots, \beta_{g}\right)$ be a canonical homology basis of $\Sigma$ realized by simple closed curves on $\Sigma$ such that $\alpha_{1}, \ldots, \alpha_{g}$ are contractible in $M_{\Sigma}$. We depict this basis in Figure 4, together with an additional curve $\alpha_{g+1}$.

Definition 2.12. For the handlebody $M_{\Sigma}$ the canonical coloured graph

$$
\mathcal{G}_{\underline{e}}^{\Sigma}=\left(c\left(\mathcal{G}_{\underline{e}}\right), \mathcal{T}_{\mathcal{G}_{\underline{e}}},\left|G \cup T_{\underline{\alpha}}\right|_{\underline{e}, \underline{x}}\right)
$$

is defined as follows. Its core is as depicted in Figure 5. The coloured graph $\left|G^{\Sigma}\right|_{\underline{e}}$ with colours $\underline{e}=\left(e_{1}, \ldots, e_{3 g-3}\right)$ for $(g>1)$ on the boundary of the tubular neighbourhood of the core is depicted in Figure 6. In addition there are the meridians $T_{\alpha_{1}}, \ldots, T_{\alpha_{3 g-3}}$ such that all lines of $\mathcal{G}_{\underline{e}}^{\Sigma}$ are lefthanded.

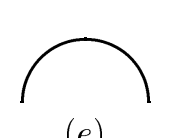

(e)

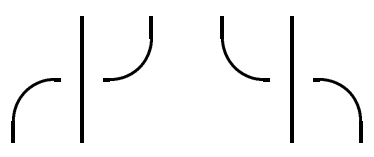

$(f)$

$(g)$

FIGURE 3. The building stones of the planar graph $|G|_{\underline{a}}$ that correspond to $\mathcal{G}_{\underline{a}} \subset S^{3}$. 


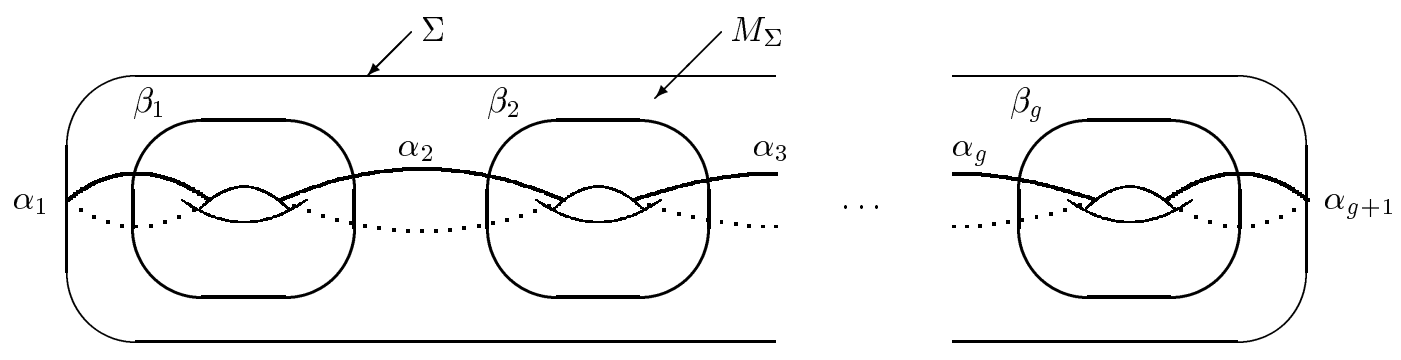

FIGURE 4. A canonical homology basis $\left(\alpha_{1}, \ldots, \alpha_{g}, \beta_{1}, \ldots, \beta_{g}\right)$ plus the additional curve $\alpha_{g+1}$.

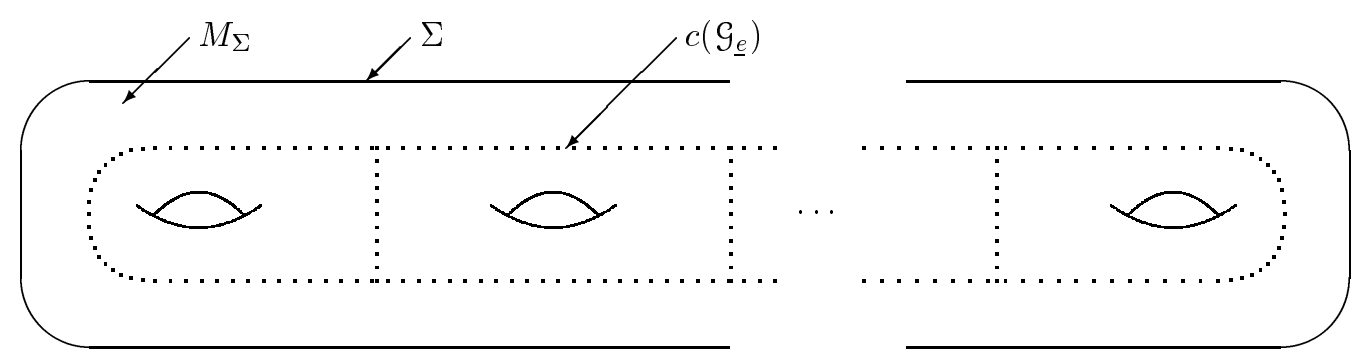

FIGURE 5. The core $c\left(\mathcal{G}_{\underline{e}}^{\Sigma}\right)$ (dotted line).

The tubular neighbourhood $\mathcal{T}_{\mathcal{G}_{e}^{\Sigma}}$ of the core is a deformation retract of $M_{\Sigma}$ and lies in a tubular neighborhood of $\Sigma=\partial M_{\Sigma}$ in $M_{\Sigma}$. On $\partial \mathcal{T}_{\mathcal{G}_{\underline{\varepsilon}}}$, which is diffeomorphic to $\Sigma^{*}$ (where ${ }^{*}$ means the opposite orientation), the family of curves $\alpha_{1}, \ldots, \alpha_{3 g-3}$ (extending the family $\left.\alpha_{1}, \ldots, \alpha_{g+1}\right)$ forms a maximal set of disjoint pairwise nonisotopic, nonintersecting smooth circles, noncontractible in $\partial \mathcal{T}_{\mathcal{G}_{e}^{\Sigma}}$ but contractible in $\mathcal{T}_{\mathcal{G}_{\underline{s}}^{\Sigma}}$. Thus they define a marking whose dual graph (see [Kohno 1992; 1994], for example) is given by Figure 5. Note that $\left|G^{\Sigma}\right|_{\underline{e}}$ is not uniquely fixed by the dual graph (whose homeomorphic image it is), but we shall agree that a given choice has been made in such a way that, for $1 \leq i \leq 3 g-3$, the line corresponding to the colour $e_{i}$ intersects the curve $\alpha_{i}$ exactly once, and intersects no other $\alpha_{j}$. Now all lines with colours $e_{1}, \ldots, e_{3 g-3}$ are assumed to be left-handed with respect to the meridians $T_{\alpha_{1}}, \ldots, T_{\alpha_{3 g-3}}$ defined by the curves $\alpha_{1}, \ldots, \alpha_{3 g-3}$.

Let $\overline{\mathcal{G}}_{\bar{f}}^{\Sigma}$ be another canonical graph in $M_{\Sigma}$ of the same form. However, now all the colours $\bar{f}=$ $\left(\bar{f}_{1}, \ldots \bar{f}_{3 g-3}\right)$ are right-handed with respect to the corresponding meridians. The tubular neighbourhoods $\mathcal{T}_{\mathcal{G}_{\underline{\underline{\varepsilon}}}^{\Sigma}}$ and $\mathcal{T}_{\overline{\mathcal{G}}_{\underline{f}}^{\Sigma}}$ may be taken to be disjoint. Alternatively, they may be identified, written as $\mathcal{T}_{\mathcal{G}^{\Sigma}}$, and the invariant (2-8) will change by a factor $w^{-2 g+2}$ only. In the following we will assume
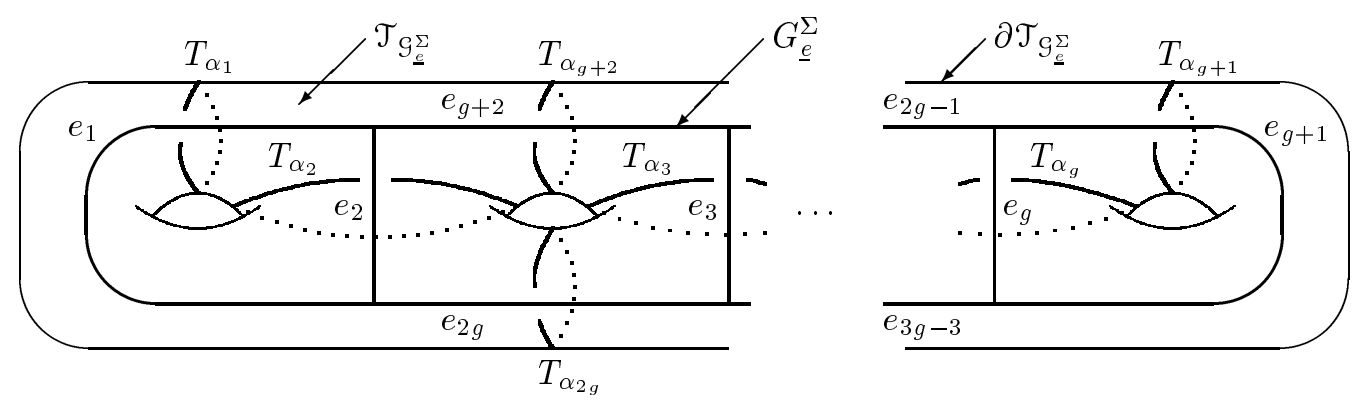

FIGURE 6. The graph $\left|G^{\Sigma}\right|_{\underline{e}}$ and the meridians $T_{\alpha_{1}}, \ldots, T_{\alpha_{3 g-3}}$. 
the second choice. For short we write the invariant of any three-manifold equipped with the canonical graph as

$$
Z\left(M\left(\mathcal{G}_{\underline{e}, \underline{\underline{f}}}^{\Sigma}\right)\right), \quad \text { where } \quad \mathcal{G}_{\underline{e}, \underline{\bar{f}}}^{\Sigma}=\mathcal{G}_{\underline{e}}^{\Sigma} \cup \overline{\mathcal{G}}_{\underline{\underline{f}}}^{\Sigma} .
$$

In particular we have

$$
Z\left(M\left(\mathcal{G}_{\underline{0}, \underline{0}}^{\Sigma}\right)\right)=w^{2-2 g} Z(M)
$$

for all colours equal to zero. Here we have used the rule for filling empty tubes given by Lemma 2.9 and Equations (2-8) and (2-10). See also Appendix A.

Finally the colours $\underline{e}$ and $\underline{\bar{f}}$ are only restricted by the fusion rules; that is, we are supposed to have

$$
\begin{aligned}
N_{e_{2} e_{g+2}}^{e_{1}} & =N_{e_{\bar{g}_{2} e_{g+3}}^{e_{3}}}^{e}=\cdots \\
& =N_{\bar{f}_{2} \bar{f}_{g+2}}^{\bar{f}_{1}}=N_{\bar{f}_{g+2} \bar{f}_{g+3}}^{\bar{f}_{3}}=\cdots=1,
\end{aligned}
$$

where $N_{j k}^{i}$ is the fusion matrix of Equation (2-3).

We introduce the handlebody $M_{\Sigma}^{*}$ with the opposite orientation, equipped with the mirror graph $\mathcal{G}_{{\underline{e^{\prime}}}^{\prime}, \underline{f}^{\prime}}^{\Sigma^{*}}$ such that gluing $M_{\Sigma}^{*}$ and $M_{\Sigma}$ along $\Sigma$ we have

$$
\left(M_{\Sigma}^{*} \backslash \mathcal{T}_{\mathcal{G}^{\Sigma^{*}}}\right) \bigcup_{\Sigma}\left(M_{\Sigma} \backslash \mathcal{T}_{\overline{\mathcal{G}} \Sigma}\right) \cong \Sigma \times I
$$

(where $I=[0,1]$ ) and

$$
\begin{aligned}
\left|G^{\Sigma^{*}}\right|_{\underline{e}^{\prime}, \underline{f}^{\prime}, \underline{x}^{\prime}}: & =\left|G^{\Sigma^{*}} \cup \bar{G}^{\Sigma^{*}} \cup T_{\underline{\alpha}^{\prime}}^{*}\right|_{\underline{\underline{e}}^{\prime}, \bar{f}^{\prime}, \underline{x^{\prime}}} \subset \Sigma \times\{0\}, \\
\left|G^{\Sigma}\right|_{\underline{e}, \underline{\bar{f}}, \underline{x}}: & :=\left|G^{\Sigma} \cup \bar{G}^{\Sigma} \cup T_{\underline{\alpha}}\right|_{\underline{e}, \underline{\bar{f}}, \underline{x}} \subset \Sigma \times\{1\},
\end{aligned}
$$

where both graphs are homotopic in $\Sigma \times[0,1]$. In Appendix A we prove the orthogonality relation

$$
\begin{aligned}
Z & \left(\left(M_{\Sigma}^{*} \bigcup_{\Sigma} M_{\Sigma}\right)\left(\mathcal{G}_{\underline{\underline{e}}^{\prime}, \underline{\underline{f}}^{\prime}}^{\Sigma^{*}} \cup \mathcal{G}_{\underline{e}, \underline{\bar{f}}}^{\Sigma}\right)\right) \\
& =\sum_{\underline{x^{\prime}}, \underline{x}} \prod_{i=1}^{3 g-3} \frac{w_{x_{i}^{\prime}}^{2}}{w^{2}} \frac{w_{x_{i}}^{2}}{w^{2}} Z\left(\Sigma \times I,\left|G^{\Sigma^{*}}\right|_{\underline{e}^{\prime}, \underline{\bar{f}}^{\prime}, \underline{x}^{\prime}} \cup\left|G^{\Sigma}\right|_{\underline{e}, \underline{\bar{f}}, \underline{x})}\right. \\
& =w^{-2 g+2} \delta_{\underline{e}^{\prime}, \underline{e}} \delta_{\bar{f}^{\prime}, \underline{\bar{f}}} \prod_{i=1}^{3 g-3} w_{e_{i}}^{-2} w_{\bar{f}_{i}}^{-2},
\end{aligned}
$$

with $\delta_{\underline{e}^{\prime}, \underline{e}}=\prod_{i=1}^{3 g-3} \delta_{e_{i}^{\prime}, e_{i}}($ for $g>1$ ) and analogously for $\delta_{\bar{f}^{\prime}, \bar{f}}$. Here and in similar situations below, we make the convention that $\mathcal{G}_{\underline{e}, \underline{f}}^{\Sigma}$ lies in $M_{\Sigma}$ and $\mathcal{G}_{\underline{\underline{\prime}}^{\prime}, \underline{f}^{\prime}}^{\Sigma^{*}}$ lies in $M_{\Sigma}^{*}$.

In the context of a topological quantum field theory, any oriented compact three-manifold $M_{2}$ with $\partial M_{2}=\Sigma$ gives rise to a vector $\left|M_{2}\right\rangle$ in a finitedimensional vector space $V^{\Sigma}$. Its dual $\left(V^{\Sigma}\right)^{*}$ is canonically isomorphic to $V^{\Sigma^{*}}$ with elements

$$
\left\langle M_{1}\right|\left(\partial M_{1}^{*} \cong \Sigma^{*}\right)
$$

such that the canonical pairing in $V^{\Sigma^{*}} \times V^{\Sigma}$ is given as

$$
\left\langle M_{1} \mid M_{2}\right\rangle=Z\left(M_{1}^{*} \underset{\Sigma}{\cup} M_{2}\right) .
$$

Furthermore, if we consider a handlebody equipped with its canonical graph, we have the maps

$$
\begin{aligned}
& \mathcal{G}_{\underline{e}, \bar{f}}^{\Sigma} \mapsto|\underline{e}, \underline{f}\rangle=w^{g-1}\left|M_{\Sigma}\left(\mathcal{G}_{\underline{e}, \bar{f}}^{\Sigma}\right)\right\rangle \in V^{\Sigma}, \\
& \mathcal{G}_{\underline{e}, \underline{f}}^{\Sigma^{*}} \mapsto\langle\underline{e}, \underline{\bar{f}}|=w^{g-1}\left\langle M_{\Sigma}\left(\mathcal{G}_{\underline{e}, \underline{f}}^{\Sigma}\right)\right| \in V^{\Sigma^{*}}
\end{aligned}
$$

such that

$$
\begin{aligned}
& \left\langle M_{1} \mid \underline{e}, \underline{\bar{f}}\right\rangle=w^{g-1} \prod_{i=1}^{3 g-3} w_{e_{i}} w_{\bar{f}_{i}} Z\left(\left(M_{1}^{*} \cup_{\Sigma} M_{\Sigma}\right)\left(\mathcal{G}_{\underline{e}, \underline{f}}^{\Sigma}\right)\right), \\
& \left\langle\underline{e}, \underline{\bar{f}} \mid M_{2}\right\rangle=w^{g-1} \prod_{i=1}^{3 g-3} w_{e_{i}} w_{\bar{f}_{i}} Z\left(\left(M_{\Sigma}^{*} \bigcup_{\Sigma} M_{2}\right)\left(\mathcal{G}_{\underline{e}, \underline{\bar{f}}}^{\Sigma^{*}}\right)\right),
\end{aligned}
$$

and by Equation (2-14)

$$
\left\langle\underline{e}^{\prime}, \underline{f}^{\prime} \mid \underline{e}, \underline{\bar{f}}\right\rangle=\delta_{\underline{e}^{\prime}, \underline{e}} \delta_{\underline{f}^{\prime}, \underline{\bar{f}}} .
$$

In particular, Equation (2-11) gives

$$
\left|M_{\Sigma}\right\rangle=w^{g-1}|\underline{0}, \underline{0}\rangle=w^{2 g-2}\left|M_{\Sigma}\left(\mathcal{G}_{\underline{0}, \underline{0}}^{\Sigma}\right)\right\rangle .
$$

One of the main results in [Karowski and Schrader 1993] was the completeness relation in terms the following surgery formula

$$
\left\langle M_{1} \mid M_{2}\right\rangle=\sum_{\underline{e} \underline{\bar{f}}}\left\langle M_{1} \mid \underline{e}, \underline{\bar{f}}\right\rangle\left\langle\underline{e}, \underline{\bar{f}} \mid M_{2}\right\rangle .
$$

In other words, the vectors $|\underline{e}, \underline{\bar{f}}\rangle$ and $\langle\underline{e}, \underline{\bar{f}}|$ form a system of dual bases in $V^{\Sigma}$ and $V^{\Sigma^{*}}$, respectively.

Remark 2.13. This completeness relation also extends to the case when $M_{1}$ and (or) $M_{2}$ contains coloured graphs (with meridians) and (or) if $M_{1}$ and $M_{2}$ contain additional boundary components along which no gluing takes place. 
Lemma E of [Karowski and Schrader 1993], combined with $(2-14)$ and the fact that $w_{i}^{*}=(-1)^{2 i} w_{i}$, shows that the antilinear extension of the map

$$
|\underline{e}, \underline{\bar{f}}\rangle \mapsto\langle\underline{e}, \underline{\bar{f}}|
$$

(for $g>1$ ) gives a map $\tau_{\Sigma}$ from $V^{\Sigma}$ onto $V^{\Sigma^{*}}$ with $\tau_{\Sigma}\left|M_{2}\right\rangle=\left\langle M_{2}\right|$ and such that the resulting hermitian form (note that $\left\langle M_{1} \mid M_{2}\right\rangle^{*}=\left\langle M_{2} \mid M_{1}\right\rangle$ ) is positive definite.

Finally, by the results in [Karowski and Schrader 1993] for the dimensions of these spaces, we have

$$
\operatorname{dim} V^{\Sigma}=\left(\operatorname{trace}\left(\sum_{a \in \mathcal{J}}\left(N^{a}\right)^{2}\right)^{g-1}\right)^{2},
$$

where the $N^{a}$ are the fusion matrices given by $(2-3)$. The square on the right-hand side of this equation also suggests the introduction of the following structure. Let $V_{l}^{\Sigma}$ and $V_{r}^{\Sigma}$ be the subspaces of $V^{\Sigma}$ spanned by elements of the form

$$
|\underline{e}\rangle:=|\underline{e}, \underline{0}\rangle \text { and }|\underline{\bar{f}}\rangle:=|\underline{0}, \underline{\bar{f}}\rangle,
$$

respectively, such that

$$
\operatorname{dim} V_{l}^{\Sigma}=\operatorname{dim} V_{r}^{\Sigma}=\operatorname{trace}\left(\vec{N}^{2}\right)^{g-1} .
$$

Then there is a canonical linear isomorphism between $V_{l}^{\Sigma} \otimes_{\mathbb{C}} V_{r}^{\Sigma}$ and $V^{\Sigma}$ by which $|\underline{e}\rangle \otimes|\underline{f}\rangle$ corresponds to $|\underline{e}, \underline{f}\rangle$. Also $V_{l(r)}^{\Sigma^{*}}=\left(V_{l(r)}^{\Sigma}\right)^{*}$. The Hilbert space structure induced by $\tau_{\Sigma}$ is obviously compatible with this isomorphism. In the next section we show that the representation of the mapping class group defined on $V^{\Sigma}$ also is compatible with this isomorphism; that is, it factorizes with respect to the left- and right-handed spaces.

\section{FINITE-DIMENSIONAL UNITARY REPRESENTATIONS OF THE MAPPING CLASS GROUP}

The aim of this section is to show that the results in [Karowski and Schrader 1993] on the Turaev-Viro theory, as outlined in Section 2, lead to a unitary representation on $V^{\Sigma}$ of the mapping class group $\mathrm{MCG}^{g}$ for $\Sigma$. It suffices to consider the case $g \geq$ 2 , since for $g=1$ the construction is well-known
[Gepner and Witten 1986; Karowski and Schrader 1993, Appendix A]. Let $\varphi$ be a diffeomorphism of $\Sigma$. We define the linear transformation $\pi(\varphi)$ on $V^{\Sigma}$ by its matrix elements

$$
\begin{aligned}
& \left\langle\underline{e}^{\prime}, \underline{f}^{\prime}|\pi(\varphi)| \underline{e}, \underline{\bar{f}}\right\rangle=w^{2 g-2} \prod_{i=1}^{3 g-3} w_{e_{i}^{\prime}} w_{e_{i}} w_{\bar{f}_{i}^{\prime}} w_{\bar{f}_{i}} \\
& \times Z\left(\left(M_{\Sigma}^{*} \underset{(\Sigma, \varphi)}{\cup} M_{\Sigma}\right)\left(\mathcal{G}_{\underline{e}^{\prime}, \underline{\bar{f}}^{\prime}}^{\Sigma^{*}} \cup \mathcal{G}_{\underline{e}, \underline{f}}^{\Sigma}\right)\right) .
\end{aligned}
$$

Here the symbol $(\Sigma, \varphi)$ means that we glue $M_{\Sigma}^{*}$ to $M_{\Sigma}$ by identifying $x \in \Sigma^{*}$ of $M_{\Sigma}^{*}$ with $\varphi^{-1}(x) \in \Sigma$ of $M_{\Sigma}$. Similarly to Equation $(2-14)$, the invariant in $(3-1)$ equals

$$
\begin{aligned}
& \sum_{\underline{x}^{\prime}, \underline{x}} \prod_{i=1}^{3 g-3} \frac{w_{x_{i}^{\prime}}^{2}}{w^{2}} \frac{w_{x_{i}}^{2}}{w^{2}} \\
& \quad \times Z\left((\Sigma \times I) \underset{(\Sigma, \varphi)}{\cup}(\Sigma \times I),\left|G^{\Sigma^{*}}\right|_{\underline{\underline{e}}^{\prime}, \overline{\underline{f}}^{\prime}, \underline{x}^{\prime}} \cup\left|G^{\Sigma}\right|_{\underline{\underline{e}}, \underline{\bar{f}}, \underline{\underline{x}}) .} .\right.
\end{aligned}
$$

In particular, by Equation $(2-14), \pi(\mathrm{id})$ is the identity transformation on $V^{\Sigma}$.

Proposition 3.1. The map $\varphi \mapsto \pi(\varphi)$ defines a representation of the diffeomorphism group of $\Sigma$. Its kernel contains the identity component; hence $\pi$ descends to a representation of the mapping class group $\mathrm{MCG}^{g}$ also denoted by $\pi$.

Proof. By the basic construction of state sums with coloured graphs in [Karowski and Schrader 1993] it is easy to see that one may move $\varphi$ to the right; that is, the partition function in (3-2) equals

$$
Z\left(\Sigma \times I,\left|G^{\Sigma^{*}}\right|_{\underline{\underline{f}}^{\prime}, \underline{\bar{f}}^{\prime}, \underline{x}^{\prime}} \cup \varphi\left(\left|G^{\Sigma}\right|_{\underline{e}, \underline{\bar{f}}, \underline{x}}\right)\right),
$$

with the following notation: $\varphi\left(\left|G^{\Sigma}\right|_{\underline{e}, \underline{\underline{f}}, \underline{x}}\right)$ is the onedimensional simplicial complex in $\Sigma \times\{1\}$ that is the image of $\left|G^{\Sigma}\right|_{\underline{e}, \underline{\bar{f}}, \underline{x}}$ under $\varphi$ and is equipped with the corresponding colours. Inserting (3-3) into (3-2) and (3-1) now proves the second part of the proposition for $\pi$, due to the homotopy invariance for coloured graphs of state sums. The 
first part of the proposition follows from the observation that

$$
\begin{aligned}
(\Sigma \times I) \underset{\left(\Sigma, \varphi_{1} \circ \varphi_{2}\right)}{\cup}( & (\Sigma \times I) \\
& \cong(\Sigma \cup I) \underset{\left(\Sigma, \varphi_{1}\right)}{\cup}(\Sigma \times I) \underset{\left(\Sigma, \varphi_{2}\right)}{\cup}(\Sigma \times I)
\end{aligned}
$$

for two diffeomorphisms $\varphi_{1}$ and $\varphi_{2}$ and from the completeness relation (2-17).

Denote by $A_{1}, \ldots, A_{3 g-3}, B_{1}, \ldots, B_{g}$ the elementary Dehn twists associated with the curves $\alpha_{1}, \ldots$, $\alpha_{3 g-3}, \beta_{1}, \ldots, \beta_{g}$ (see Figures 4 and 6 ). Then the mapping class group $\mathrm{MCG}^{g}$ is generated by the elements $A_{1}, \ldots, A_{g}, A_{g+2}, B_{1}, \ldots, B_{g}$ (see [Wajnryb 1983], for example). Set

$$
\begin{aligned}
& S_{i}=A_{2 g-2+i}^{-1} B_{i} A_{2 g-2+i}^{-1} \quad \text { for } 1<i<g \\
& S_{1}=A_{1}^{-1} B_{1} A_{1}^{-1} \\
& S_{g}=A_{g+1}^{-1} B_{g} A_{g+1}^{-1} .
\end{aligned}
$$

Then $\mathrm{MCG}^{g}$ is also generated by $A_{1}, \ldots, A_{3 g-3}$, $S_{1}, \ldots, S_{g}$.

Proposition 3.2. The representation $\pi(\varphi)$ of $\mathrm{MCG}^{g}$ defined by (3-1) satisfies

$$
\left\langle\underline{e}^{\prime}, \underline{f}^{\prime}|\pi(\varphi)| \underline{e}, \underline{\bar{f}}\right\rangle=\left\langle\underline{e}^{\prime}\left|\pi_{l}(\varphi)\right| \underline{e}\right\rangle\left\langle\underline{f}^{\prime}\left|\pi_{r}(\varphi)\right| \underline{\bar{f}}\right\rangle .
$$

This means $\pi$ decomposes into two maps $\pi_{l}$ and $\pi_{r}$ on $V_{l}^{\Sigma}$ and $V_{r}^{\Sigma}$, respectively, defined by (2-18). By use of the canonical isomorphism $V^{\Sigma} \cong V_{l}^{\Sigma} \otimes_{\mathbb{C}} V_{r}^{\Sigma}$, Equation (3-4) implies that

$$
\pi(\varphi) \cong \pi_{l}(\varphi) \otimes \pi_{r}(\varphi) .
$$

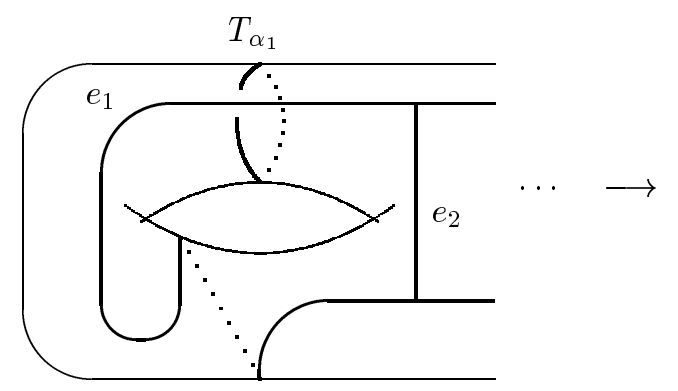

Furthermore the map $\pi_{r}$ is complex conjugate to the map $\pi_{l}$ in these bases. For the generating elements $A_{1}, \ldots, A_{3 g-3}, S_{1}, \ldots, S_{g}$ of $\mathrm{MCG}^{g}$ the map $\pi_{l}$ is given by

$$
\begin{aligned}
& \left\langle\underline{e}^{\prime}\left|\pi_{l}\left(A_{i}\right)\right| \underline{e}\right\rangle=\left\langle\underline{e}^{\prime}, \underline{0}\left|\pi\left(A_{i}\right)\right| \underline{e}, \underline{0}\right\rangle, \\
& \left\langle\underline{e}^{\prime}\left|\pi_{l}\left(S_{i}\right)\right| \underline{e}\right\rangle=w^{-1}\left\langle\underline{e}^{\prime}, \underline{0}\left|\pi\left(S_{i}\right)\right| \underline{e}, \underline{0}\right\rangle,
\end{aligned}
$$

where $w=\sqrt{r / 2} /(\sin \pi / r)$ (see Equation $(2-9))$.

Proof. By explicit calculation of the matrix elements (3-1) we prove the factorization property (3-4) for the generators of $\mathrm{MCG}^{g}$. Thus it suffices to define $\pi_{l}$ on these elements. We start with the elements $A_{1}, \ldots, A_{3 g-3}$ and determine $\pi\left(A_{1}\right)|\underline{e}, \underline{0}\rangle$ first. For the elementary Dehn twist $A_{1}$ along $\alpha_{1}$, the graph $\left|G^{\Sigma}\right|_{\underline{e}, \underline{0}, \underline{x}}$ on $\partial \mathcal{T}_{\mathcal{G}^{\Sigma}}$ of Figure 6 is changed into $A_{1}\left|G^{\Sigma}\right|_{\underline{e}, \underline{0}, \underline{x}}$ as given in Figure 7.

We see that the meridians $T_{\alpha_{1}}, \ldots, T_{\alpha_{3 g-3}}$ are left unchanged. Also the equivalence of the two pictures in Figure 7 on the level of state sums results from [Karowski and Schrader 1993, Lemma 5.4] (compare also the proof of Theorem 2.11). Hence we obtain by Equations (2-5) and (2-6)

$$
\pi\left(A_{1}\right)|\underline{e}, \underline{0}\rangle=|\underline{e}, \underline{0}\rangle q_{e_{1}}^{2},
$$

where $q_{j}=(-1)^{-j} q^{j(j+1)}$ for $j \in \mathcal{J}$. For righthanded lines we would obtain a similar picture as that of Figure 7, except that the self-overcrossing is replaced by the opposite version. Therefore we obtain

$$
\pi\left(A_{1}\right)|\underline{e}, \underline{\bar{f}}\rangle=|\underline{e}, \underline{\bar{f}}\rangle q_{e_{1}}^{2} q_{\bar{f}_{1}}^{-2} .
$$

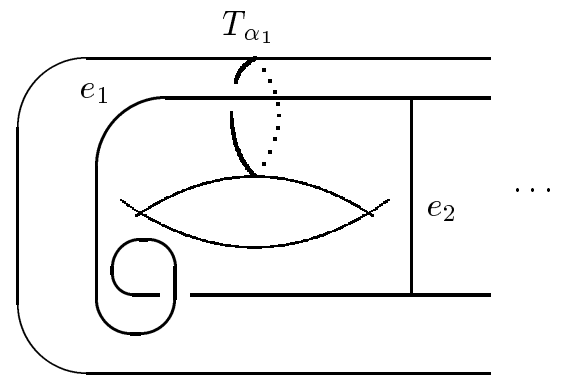

FIGURE 7. The Dehn twist $A_{1}$ applied to $\left|G^{\Sigma}\right|_{\underline{e}, \underline{0}, \underline{x}}$. 
The discussion for any $A_{i}(1 \leq i \leq 3 g-3)$ is analogous, giving

$$
\pi\left(A_{i}\right)|\underline{e}, \underline{\bar{f}}\rangle=|\underline{e}, \underline{\bar{f}}\rangle q_{e_{i}}^{2} q_{\bar{f}_{i}}^{-2}
$$

for $1 \leq i \leq 3 g-3$.

We turn to the investigation of $S_{1}=A_{1}^{-1} B_{1} A_{1}^{-1}$ and discuss first $\pi\left(S_{1}\right)|\underline{e}, \underline{0}\rangle$. The graph $\left|G^{\Sigma}\right|_{\underline{e}}$ and the meridians $T_{\alpha_{2}}, \ldots, T_{\alpha_{3 g-3}}$ do not change under $B_{1}$. The circle $B_{1} T_{\alpha_{1}}$ takes the form depicted in Figure 8. This results in the following action of $S_{1}$ on $\left|G^{\Sigma}\right|_{\underline{e}}$ and its meridians as depicted in Figure 9. In particular the circle $A_{1}^{-1} B_{1} A_{1}^{-1} T_{\alpha_{1}}$ is just $\beta_{1}$. To evaluate the matrix elements $\left\langle\underline{e}^{\prime}, \underline{f}^{\prime}\left|\pi\left(S_{1}\right)\right| \underline{e}, \underline{f}\right\rangle$

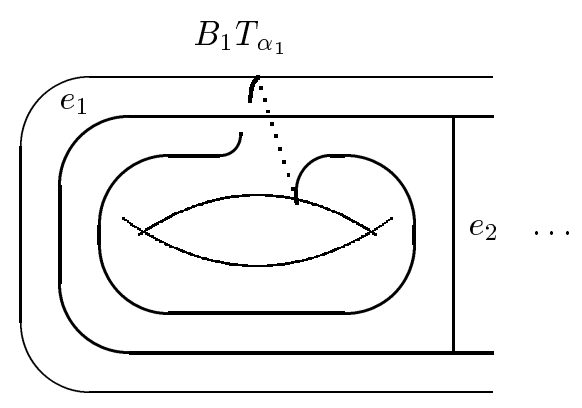

FIGURE 8. The effect of $B_{1}$ on $\left|G^{\Sigma}\right|_{\underline{e}, \underline{0}, \underline{x}}$. we apply a basis transformation $|\underline{e}, \underline{0}\rangle \mapsto|\underline{\tilde{e}}, \underline{0}\rangle$. First we omit the meridian $T_{\alpha_{2}}$ of $e_{2}$ by Equation (2-10). Then we use the following identity for the coloured graph (see [Karowski and Schrader 1993, (4.11)], a special case of a Fierz transformation):

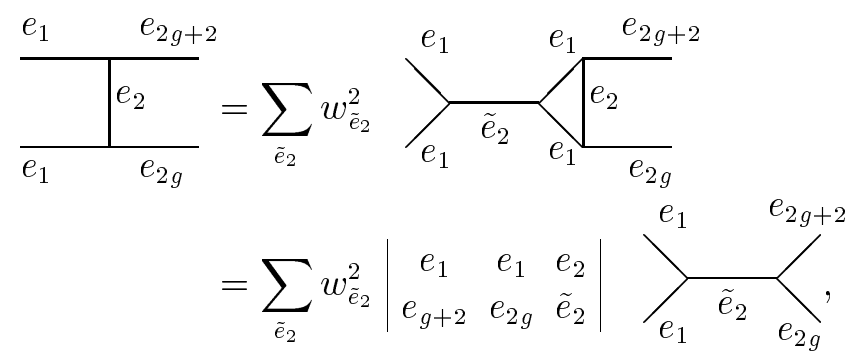

where $\left|\begin{array}{lll}a & b & c \\ d & e & f\end{array}\right|$ is the $6 j$-symbol of Equation (2-4). We obtain the transformation matrix

$$
\langle\underline{\tilde{e}}, \underline{\underline{0}} \mid \underline{e}, \underline{0}\rangle=\prod_{i \neq 2} \delta_{\tilde{e}_{i} e_{i}} w_{\tilde{e}_{2}} w_{e_{2}}\left|\begin{array}{ccc}
e_{1} & e_{1} & e_{2} \\
e_{g+2} & e_{2 g} & \tilde{e}_{2}
\end{array}\right| .
$$

We also apply the analogous transformation

$$
\left\langle\underline{e}^{\prime}, \underline{0}\right| \mapsto\left\langle\underline{e}^{\prime}, \underline{0}\right| .
$$

Using the cutting rules established in [Karowski and Schrader 1993] (see also Appendix A) to
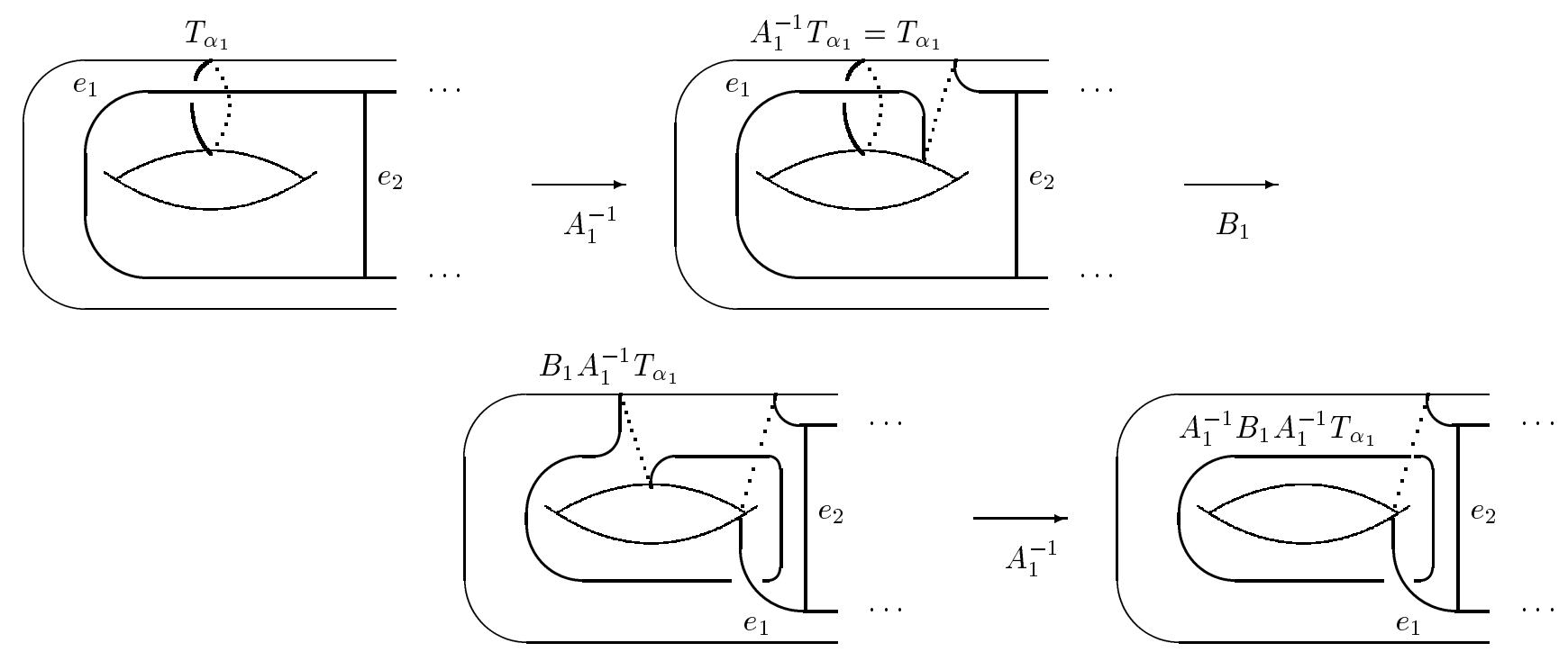

FIGURE 9. The effect of $S_{1}=A_{1}^{-1} B_{1} A_{1}^{-1}$ on $\left|G^{\Sigma}\right|_{\underline{e}, \underline{\underline{0}}, \underline{x}}$. 
Equations (3-1) and (3-2) for $\varphi=S_{1}$ we obtain the resulting expression

$$
\begin{aligned}
& \left\langle\underline{\tilde{e}}^{\prime}, \underline{0}\left|\pi\left(S_{1}\right)\right| \underline{\tilde{e}}, \underline{0}\right\rangle \\
& =w^{2 g-2} \prod_{i=1}^{3 g-3} w_{\tilde{e}_{i}^{\prime}} w_{\tilde{e}_{i}} \sum_{\underline{x}^{\prime}, \underline{x}} \prod_{i=1}^{3 g-3} \frac{w_{x_{i}^{\prime}}^{2}}{w^{2}} \frac{w_{x_{i}}^{2}}{w^{2}} \\
& \times Z\left(M_{1},\left|G_{1}\right|\right) w^{2} \frac{\delta_{\tilde{e}_{2}^{\prime} \tilde{e}_{2}}}{w_{\tilde{e}_{2}}^{2}} Z\left(M_{2},\left|G_{2}\right|\right),
\end{aligned}
$$

where $Z\left(M_{1},\left|G_{1}\right|\right)$ is the invariant of the manifold and the coloured graph depicted in Figure 10 and $Z\left(M_{2},\left|G_{2}\right|\right)$ that of the remainder. Here $x_{1}$ is the colour of $S_{1} T_{\alpha_{1}}$ belonging to the line of colour $\tilde{e}_{1}$ on the inner part of the surface and $x_{1}^{\prime}$ is the colour of the meridian for $\tilde{e}_{1}^{\prime}$ on the outer part of the surface.

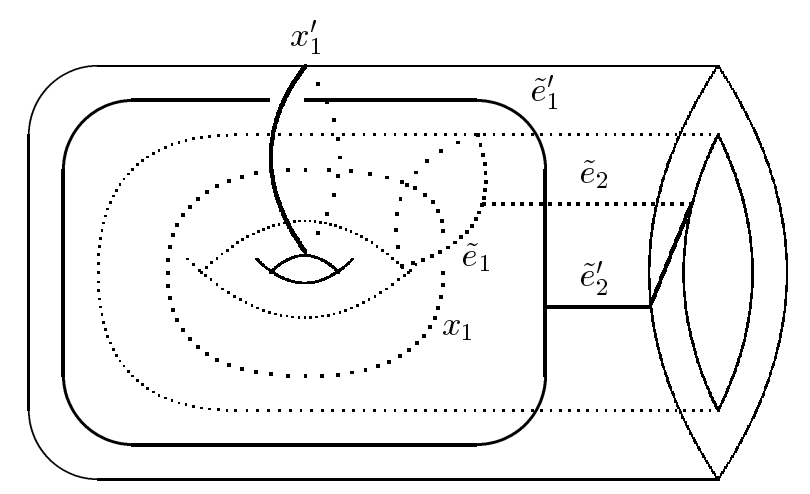

FIGURE 10. Graphical description for the nontrivial contribution to the matrix element $\left\langle\underline{\tilde{e}}^{\prime}, \underline{0}\left|\pi\left(S_{1}\right)\right| \underline{\tilde{e}}, \underline{0}\right\rangle$.

By appropriate cuttings (in analogy to the proof of (2-14) in Appendix A) we write $Z\left(M_{1},\left|G_{1}\right|\right)$ and $Z\left(M_{2},\left|G_{2}\right|\right)$ in terms of invariants of planar graphs and find

$$
\left\langle\underline{\tilde{e}}^{\prime}, \underline{0}\left|\pi\left(S_{1}\right)\right| \underline{\tilde{e}}, \underline{0}\right\rangle=\frac{1}{w} S_{\tilde{e}_{1}^{\prime} \tilde{e}_{1}}^{\tilde{e}^{2}} \prod_{i=2}^{3 g-3} \delta_{\tilde{e}_{i}^{\prime}} \tilde{e}_{i}
$$

where

$$
S_{\tilde{e}_{1}^{\prime}, \tilde{e}_{1}}^{\tilde{e}_{2}}=\frac{w_{\tilde{e}_{1}^{\prime}} w_{\tilde{e}_{1}}}{w} Z\left(\tilde{e}_{\tilde{e}_{1}^{\prime}} \tilde{e}_{2}\right.
$$

in the sense of a state sum of a planar graph on $S^{2}$ [Karowski and Schrader 1993, Example 4.10]. For right lines the matrix $S$ in Equation $(3-10)$ is to be replaced by the complex conjugate matrix.

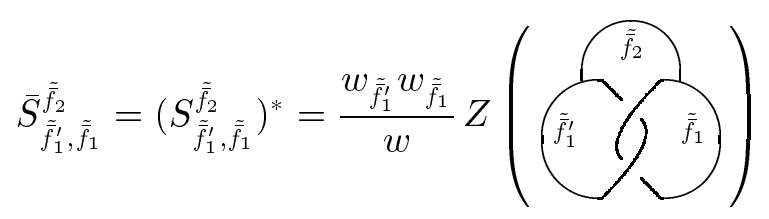

By the same method as above one can calculate the general matrix element

$$
\left\langle\underline{\tilde{e}}^{\prime}, \underline{\bar{f}}^{\prime}\left|\pi\left(S_{1}\right)\right| \underline{\tilde{e}}, \underline{\bar{f}}\right\rangle=S_{\tilde{\epsilon}_{1}^{\prime} \tilde{e}_{1}}^{\tilde{e}_{2}} \bar{S}_{\tilde{\bar{f}}_{1}^{\prime}, \tilde{f}_{1}}^{\tilde{f}_{2}} \prod_{i=2}^{3 g-3} \delta_{\tilde{e}_{i}^{\prime} \tilde{e}_{i}} \delta_{\tilde{f}_{i}^{\prime}} \tilde{\bar{f}}_{i} .
$$

In terms of the original basis we obtain, by the transformation formula (3-9),

$$
\begin{aligned}
\left\langle\underline{e}^{\prime}, \underline{f}^{\prime}\right| \pi & \left(S_{1}\right)|\underline{e}, \underline{\bar{f}}\rangle \\
& =S_{e_{1}^{\prime} e_{2}^{\prime}, e_{1} e_{2}}^{e_{g+2}, e_{2 g}} \bar{S}_{\bar{f}_{1}^{\prime} \bar{f}_{2}^{\prime}, \bar{f}_{1} \bar{f}_{2}}^{\bar{f}_{2+2}, \bar{f}_{2 g}} \prod_{j=3}^{3 g-3} \delta_{e_{j}^{\prime} e_{j}} \delta_{\bar{f}_{i}^{\prime} \bar{f}_{i}},
\end{aligned}
$$

where

$S_{e_{1}^{\prime} e_{2}^{\prime}, e_{1} e_{2}}^{e_{g+2}, e_{2 g}}$

$$
=w_{e_{2}^{\prime}} w_{e_{2}} \sum_{\tilde{e}_{2}} w_{\tilde{e}_{2}}^{2}\left|\begin{array}{ccc}
e_{1}^{\prime} & e_{1}^{\prime} & e_{2}^{\prime} \\
e_{g+2} & e_{2 g} & \tilde{e}_{2}
\end{array}\right|\left|\begin{array}{ccc}
e_{1} & e_{1} & e_{2} \\
e_{g+2} & e_{2 g} & \tilde{e}_{2}
\end{array}\right| S_{e_{1}^{\prime} e_{1}}^{\tilde{e}_{2}}
$$$$
=\frac{w_{e_{1}^{\prime}} w_{e_{2}^{\prime}} w_{e_{1}} w_{e_{2}}}{w} \sum_{\tilde{e}_{2}} w_{\tilde{e}_{2}}^{2} Z\left(e_{1}^{e_{1}^{\prime}}\right.
$$

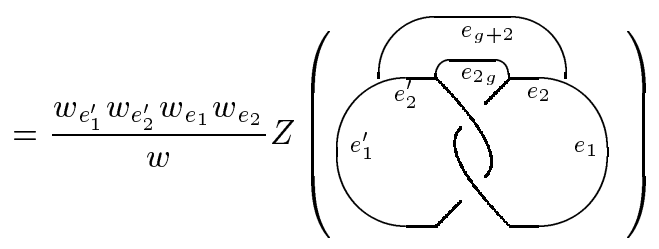

(we have again made use of a Fierz transformation in the proof of this last equality) and $\bar{S}=S^{*}$. 
Obviously $\pi\left(S_{g}=A_{g+1}^{-1} B_{g} A_{g+1}^{-1}\right)$ may be calculated in the same way giving (compare Figure 6 ):

$$
\begin{aligned}
& \left\langle\underline{e}^{\prime}, \bar{f}^{\prime}\left|\pi\left(S_{g}\right)\right| \underline{e}, \overline{\bar{f}}\right\rangle \\
& =S_{e_{g+1}^{\prime} e_{g}^{\prime}, e_{g+1} e_{g}}^{e_{3 g-3}, e_{2 g-1}} \overline{\bar{S}}_{\bar{f}_{g+1}^{\prime} \bar{f}_{g-3}, \bar{f}_{2 g-1}^{\prime}, \bar{f}_{g+1} \bar{f}_{g}} \prod_{\substack{i=1 \\
i \neq g, g+1}} \delta_{e_{j}^{\prime} e_{j}} \delta_{\bar{f}_{i}^{\prime} \bar{f}_{i}} .
\end{aligned}
$$

It remains to calculate the matrix elements of

$$
\pi\left(S_{i}=A_{2 g-2+i}^{-1} B_{i} A_{2 g-2+i}^{-1}\right)
$$

for $1<i<g$. We look at the local picture around the $i$-th hole; see Figure 11, where the colours $e_{\mathrm{I}}, e_{\mathrm{II}}, e_{\mathrm{III}}$ and $e_{\mathrm{IV}}$ are

$$
\begin{aligned}
e_{\mathrm{I}}=e_{g+i-1} & \text { if } i>2, \\
e_{\mathrm{II}}=e_{2 g-3+i} & \text { if } i>2, \\
e_{\mathrm{I}}=e_{\mathrm{II}}=e_{1} & \text { if } i=2, \\
e_{\mathrm{III}}=e_{g+i+1} & \text { if } i<g-1, \\
e_{\mathrm{IV}}=e_{2 g-i+1} & \text { if } i<g-1, \\
e_{\mathrm{III}}=e_{\mathrm{IV}}=e_{g+1} & \text { if } i=g-1 .
\end{aligned}
$$

By methods similar to those used to calculate the matrix elements of $S_{1}$ we obtain

$$
\begin{aligned}
& \left\langle\underline{e}^{\prime}, \underline{\bar{f}}^{\prime}\left|\pi\left(S_{i}\right)\right| \underline{e}, \underline{\bar{f}}\right\rangle \\
& =\frac{1}{w^{2}} \prod_{j \in I_{1}} w_{e_{j}^{\prime}} w_{e_{j}} w_{\bar{f}_{j}^{\prime}} w_{\bar{f}_{j}} \prod_{j \in I_{2}} \delta_{e_{j}^{\prime} e_{j}} \delta_{\bar{f}_{j}^{\prime} \bar{f}_{j}} Z_{i} \cdot \bar{Z}_{i}
\end{aligned}
$$

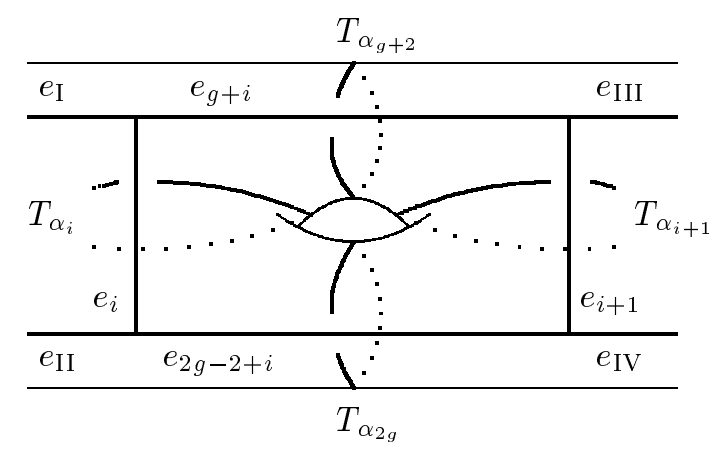

FIGURE 11. The local form of $\left|G^{\Sigma}\right|_{\underline{e}}$ and its meridians around the $i$-th hole. for $1<i<g$, where

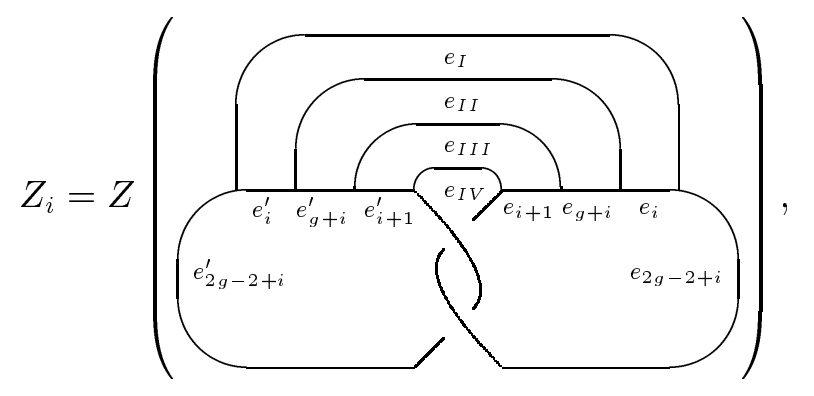

$\bar{Z}_{i}=Z_{i}^{*}, I_{1}=\{2 g-2+i, i, g+i, i, i+1\}$ and $I_{2}=$ $\{1, \ldots, 3 g-3\} \backslash I_{1}$. This concludes the proof of Proposition 3.2.

Before we prove unitarity of the representation of the mapping class group given by the invariants of graphs on Riemann surfaces we discuss some properties of two special $(r-1) \times(r-1)$-matrices: the diagonal matrix $A$ (see Equation (3-7))

$$
A_{a b}=\delta_{a b} q_{a}^{2}
$$

and $S^{c}$ (for fixed $c$ ) with matrix elements given by Equation (3-11)

$$
S_{a b}^{c}=\frac{q_{a}^{-2} q_{b}^{-2}}{w} \sum_{d} w_{d}^{2} q_{d}^{2} N_{a b}^{c d}
$$

where $N_{a b}^{c d}=w_{a} w_{b}\left|\begin{array}{lll}a & b & d \\ b & a & c\end{array}\right|$. This representation in terms of a $6 j$-symbol and the following relations are proved in Appendix B. Note that for $c=0$ this formula coincides with the representation of $S_{a b}$ (A.1) in [Karowski and Schrader 1993] for the genus $g=1$ case, i.e., $S^{0}=S$, because $N^{0 d}=$ $N^{d}$ is the fusion matrix $(2-3)$. The matrix $S^{c}$ is symmetric and $q_{c}^{-1 / 2} S^{c}$ is real:

$$
S_{a b}^{c}=S_{b a}^{c}=q_{c}\left(S_{a b}^{c}\right)^{*} .
$$

Moreover the matrices $A$ and $S^{c}$ are unitary:

$$
A^{\dagger} A=1, \quad S^{c}\left(S^{c}\right)^{\dagger}=1^{c} .
$$

In the second relation $1^{c}$ has to be understood as the unit matrix with indices $a$ subject to the condition $N_{a a}^{c} \neq 0$. We also have

$$
A S^{c} A S^{c} A=\kappa S^{c}
$$


where

$$
\kappa=\frac{1}{w} \sum_{d} w_{d}^{4} q_{d}^{2}=q^{-3 / 2} e^{-i \pi(1 / 4+r / 2)}
$$

(This phase factor $\kappa$ differs from the one in [Reshetikhin and Turaev 1991] and [Kirby and Melvin 1991] since we have a different sign convention for $q_{a}^{2}$.) The matrix $N^{c d}$ (for $c$ and $d$ fixed) with matrix elements $N_{a b}^{c d}$ is diagonalized by $S^{c}$ :

$$
\left(S^{c}\right)^{-1} N^{c d} S^{c}=\operatorname{diag}\left(S_{d b} / S_{0 b}\right)_{b \in \mathcal{J}} .
$$

The eigenvalues of $N^{c d}$ are independent of $c$, implying that $N^{c d}$ is equivalent to the fusion matrix $N^{d}$ :

$$
\left(S^{c} S^{-1}\right)^{-1} N^{c d} S^{c} S^{-1}=N^{d} .
$$

That $A$ is unitary and $S^{c}$ is symmetric is obvious; the other relations are proved in Appendix B.

By Proposition 3.1 we now have:

Theorem 3.3. The representation of the mapping class group $\varphi \mapsto\left\langle\underline{e}^{\prime}, \underline{f}^{\prime}|\pi(\varphi)| \underline{e}, \underline{\bar{f}}\right\rangle$ on $V^{\Sigma}$ (see $(3-1))$ is unitary.

Proof. Since $\mathrm{MCG}^{g}$ is generated by $A_{1}, \ldots, A_{g}, A_{g+1}$ and $S_{1}, \ldots, S_{g}$, Equations (3-19) imply unitarity because of Equations (3-8), (3-13), (3-14), (3-15) and transformations like (3-9).

Moreover, because of the decomposition formula of Proposition 3.2, we have:

Theorem 3.4. The map $\varphi \mapsto\left\langle\underline{e}^{\prime}\left|\pi_{l}(\varphi)\right| \underline{e}\right\rangle$ on $V_{l}^{\Sigma}$ and the map

$$
\varphi \mapsto\left\langle\underline{\bar{f}}^{\prime}\left|\pi_{r}(\varphi)\right| \underline{\bar{f}}\right\rangle
$$

on $V_{r}^{\Sigma}$ (see Equation (3-6)) both define a unitary ray representation of $\mathrm{MCG}^{g}$ such that $\pi=\pi_{l} \otimes \pi_{r}$ and $\pi_{r}=\pi_{l}^{*}$. For genus $g=1$ the maps $\rho_{l}(\varphi)$ and $\rho_{r}(\varphi)$ given by $\rho_{l}(S)=\pi_{l}(S)$ and $\rho_{l}(A)=$ $\kappa^{-1 / 3} \pi_{l}(A)$ for the generators and $\rho_{r}=\rho_{l}^{*}$ define representations of $\mathrm{MCG}^{1}$.

Proof. It remains to prove that the relations $S^{4}=$ 1 and $A S A S A=S$ for the generators of $\mathrm{MCG}^{1}$ also hold for the representation matrices $\rho_{l}$. This follows from Equations (3-19)-(3-21). Thus we have proved the main results of this section.

The preceding decomposition of the representation $\pi$ into $\pi_{l}$ and $\pi_{r}$ provides a choice of the phases that makes them into ray representations only. For $g=1$ the phases have been adjusted in such a way that one indeed obtains representations $\rho_{l}$ and $\rho_{r}$.

Example 3.5. If $\varphi(A, S)$ is a word composed of $A$ 's and $S$ 's then

$$
\left\langle\underline{e}^{\prime}\left|\pi_{l}(\varphi(A, S))\right| \underline{e}\right\rangle=w^{N_{S}}\left\langle\underline{e}^{\prime}, \underline{0}|\pi(\varphi(A, S))| \underline{e}, \underline{0}\right\rangle,
$$

where $N_{S}$ is the number of $S$ 's in $\varphi(A, S)$.

Example 3.6. For genus $g=1$ we have

$$
\begin{aligned}
\left\langle e^{\prime}\left|\pi_{l}\left(A^{m_{t}} S A^{m_{t-1}} S \cdots A^{m_{1}} S\right)\right| e\right\rangle & \\
& =\left(A^{m_{t}} S A^{m_{t-1}} S \cdots A^{m_{1}} S\right)_{e^{\prime} e}
\end{aligned}
$$

and

$$
\begin{aligned}
& \left\langle e^{\prime}\left|\pi_{l}\left(S A^{m_{t}} S A^{m_{t-1}} S \cdots A^{m_{1}} S\right)\right| e\right\rangle \\
& =\left(S A^{m_{t}} S A^{m_{t-1}} S \cdots A^{m_{1}} S\right)_{e^{\prime} e}
\end{aligned}
$$

where the matrix $A$ is given by Equation (3-16) and $S$, given by Equation $(3-17)$ for $c=0$, is the Verlinde matrix

$$
S_{a b}=(-1)^{2 a+2 b} \frac{\sin (\pi / r)(2 a+1)(2 b+1)}{w \sin (\pi / r)} .
$$

\section{APPLICATION TO THE PARTITION FUNCTION OF ARBITRARY MANIFOLDS}

Based on our discussion in Section 3 of the representation of the mapping class group, we now provide a formulation on how to calculate the TuraevViro state sum $Z(M)$ for an arbitrary oriented compact three-manifold $M$. In particular we give an explicit formula for manifolds obtained from a figure-eight knot by an $(-n / m)$-surgery. Let $M_{\Sigma}$ be an arbitrary handlebody with boundary $\Sigma$ of genus $g$, viewed as embedded in $S^{3}$. We set $\tilde{M}_{\Sigma}^{*}=$ $S^{3} \backslash M_{\Sigma}$ with $\partial \tilde{M}_{\Sigma}=\Sigma$, such that $\tilde{M}_{\Sigma}^{*} \cup_{\Sigma} M_{\Sigma}=S^{3}$. Let $\varphi$ be an arbitrary orientation preserving diffeomorphism and set $M=\tilde{M}_{\Sigma}^{*} \cup_{(\Sigma, \varphi)} M_{\Sigma}$. Any three-manifold can be obtained (nonuniquely) in 
this way [Heegaard 1916]. By our previous discussion we may write the Turaev-Viro invariant of the three-manifold $M$ as

$$
\begin{aligned}
Z(M, r) & =\left\langle\tilde{M}_{\Sigma}|\pi(\varphi)| M_{\Sigma}\right\rangle \\
& =\sum_{\underline{e}, \underline{\bar{f}}}\left\langle\tilde{M}_{\Sigma} \mid \underline{e}, \underline{\bar{f}}\right\rangle\left\langle\underline{e}, \underline{\bar{f}}|\pi(\varphi)| M_{\Sigma}\right\rangle .
\end{aligned}
$$

In particular, for $\varphi=1$, we get of course

$$
\begin{aligned}
Z\left(S^{3}, r\right) & =\left\langle\tilde{M}_{\Sigma} \mid M_{\Sigma}\right\rangle \\
& =\left\langle\tilde{M}_{\Sigma} \mid \underline{0}, \underline{0}\right\rangle\left\langle\underline{0}, \underline{0} \mid M_{\Sigma}\right\rangle=w^{-2} .
\end{aligned}
$$

Because of $(2-16)$ this means that $\left\langle\underline{0}, \underline{0} \mid M_{\Sigma}\right\rangle=$ $w^{g-1}$ and $\left\langle\tilde{M}_{\Sigma} \mid \underline{0}, \underline{0}\right\rangle=w^{-g-1}$. We want to factorize the right-hand side of Equation (4-1) into a left- and a right-handed contribution. The factorization of the first factor,

$$
\begin{aligned}
\left\langle\tilde{M}_{\Sigma} \mid \underline{e}, \underline{\bar{f}}\right\rangle & =w^{g+1}\left\langle\tilde{M}_{\Sigma} \mid \underline{e}, \underline{0}\right\rangle\left\langle\tilde{M}_{\Sigma} \mid \underline{0}, \underline{\bar{f}}\right\rangle \\
& =w^{g+1}\left\langle\tilde{M}_{\Sigma} \mid \underline{e}\right\rangle\left\langle\tilde{M}_{\Sigma} \mid \underline{f}\right\rangle,
\end{aligned}
$$

follows from the trivial braiding of left- and righthanded lines that was proved in [Karowski and Schrader 1993], which implies that the graphs $\mathcal{G}_{\underline{\underline{e}}}^{\Sigma}$ and $\mathcal{G}_{f}^{\Sigma}$ may be moved into two nonintersecting balls $\bar{D}^{3} \subset S^{3}$, respectively. The normalization follows from the definitions $(2-15)$ and the equalities $Z\left(S^{3}\right)=w^{-2}$ and $\left\langle\tilde{M}_{\Sigma} \mid \underline{0}, \underline{0}\right\rangle=w^{1-g}\left\langle\tilde{M}_{\Sigma} \mid M_{\Sigma}\right\rangle=$ $w^{-1-g}$. We have introduced $\left\langle\tilde{M}_{\Sigma} \mid \underline{e}\right\rangle=\left\langle\tilde{M}_{\Sigma} \mid \underline{e}, \underline{0}\right\rangle$ such that $\left\langle\tilde{M}_{\Sigma} \mid \underline{0}\right\rangle=w^{-g-1}$. The factorization of the second factor,

$$
\left\langle\underline{e}, \underline{\bar{f}}|\pi(\varphi)| M_{\Sigma}\right\rangle=w^{g-1}\left\langle\underline{e}\left|\pi_{l}(\varphi)\right| \underline{0}\right\rangle\left\langle\underline{\bar{f}}\left|\pi_{r}(\varphi)\right| \underline{0}\right\rangle,
$$

follows from Proposition 3.2 and Equation (2-16). Therefore we have

$$
Z(M, r)=Z_{l}(M, r) \cdot Z_{r}(M, r),
$$

where

$$
\begin{aligned}
& Z_{l}(M, r) \\
& \quad=e^{i \psi\left(\tilde{M}_{\Sigma}, \varphi\right)} w^{g} \sum_{\underline{e}}\left\langle\tilde{M}_{\Sigma} \mid \underline{e}\right\rangle\left\langle\underline{e}\left|\pi_{l}(\varphi)\right| \underline{0}\right\rangle
\end{aligned}
$$

and $Z_{r}(M, r)=Z_{l}^{*}(M, r)$. For $\varphi=1$ we have

$$
Z_{l}\left(S^{3}, r\right)=e^{i \psi} w^{-1}
$$

compare Equation (4-2). The phase $\psi\left(\tilde{M}_{\Sigma}, \varphi\right)$, which is of course not determined by the TuraevViro invariant, has to be chosen in such a way that $Z_{l}(M, r)$ is invariant (see below).

We now discuss the case where $\Sigma$ is a set of (possibly knotted) tori:

$$
\Sigma=\bigcup_{i=1}^{N}\left(S^{1} \times S^{1}\right)_{i},
$$

forming a link $\mathcal{L}$ with $N$ components. For simplicity we write $\left\langle\tilde{M}_{\Sigma}\right|=\langle\mathcal{L}|$ and use $\left|M_{\Sigma}\right\rangle=|\underline{0}, \underline{0}\rangle$. The mapping class group of a torus is generated by the elements $S$ and $A$ with the relations $S^{4}=1$ and $(S A)^{3}=S^{2}$. We use the representation as given in [Karowski and Schrader 1993] and Theorem 3.4 (see also [Gepner and Witten 1986]). If we consider the map $\varphi=A_{1}^{n_{1}} \cdots A_{N}^{n_{N}} S_{1} \cdots S_{N}$, then instead of the invariant (4-3) we get the following formula, due to Reshetikhin and Turaev [1991] (see also [Kirby and Melvin 1991]).

Theorem 4.1. Let $\mathcal{L}$ be a link of $N$ components with framing $n_{1}, \ldots, n_{N}$. Then

$$
\begin{aligned}
& Z_{l}(M, r)=\kappa^{-\sigma_{\mathcal{L}}} w^{1-N} \\
& \quad \times \sum_{\underline{e}}\left\langle\mathcal{L}\left|\pi_{l}\left(A_{1}^{n_{1}} \cdots A_{N}^{n_{N}}\right)\right| \underline{e}\right\rangle w_{e_{1}}^{2} \cdots w_{e_{N}}^{2}
\end{aligned}
$$

is invariant under Kirby moves, if the phase factor

$$
\left.\kappa=q^{-3 / 2} e^{-i \pi(1 / 4+r / 2}\right)
$$

is given by Equation (3-21) and $\sigma_{\mathcal{L}}$ is the signature of the linking matrix. Therefore $Z_{l}(M, r)$ is an invariant of the three-manifold obtained by surgery along $\mathcal{L}$.

Note that (4-4) for $N=1$ is compatible with (4-3) for $g=1$.

Proof. Let $\mathcal{L}^{\prime}$ be the link obtained by a Kirby move at a line of $\mathcal{L}$ of colour $e$. We use Theorem 2.11 to write the invariant of the link $\mathcal{L}^{\prime}$ embedded in $S^{3}$ as an invariant of a planar graph. We then 
apply formula (B-4) of Appendix B and use again Theorem 2.11 in the inverse direction to obtain

$$
\begin{aligned}
& \sum_{e^{\prime}} w_{e^{\prime}}^{2}\left\langle\mathcal{L}^{\prime}\left|\pi_{l}\left(\varphi^{\prime}\right)\right| \underline{e}, e^{\prime}\right\rangle \\
& =\sum_{e^{\prime}} w_{e^{\prime}}^{2} Z(\cdots= \\
& =w^{\chi-2} \sum_{e^{\prime}} w_{e^{\prime}}^{2} Z\left(\stackrel{\cdots}{e} \cdots \bigcap_{e^{\prime}}\right) \\
& =w^{\chi-2} w \kappa Z\left(\begin{array}{l}
\ldots e \\
\ldots
\end{array}\right)
\end{aligned}
$$

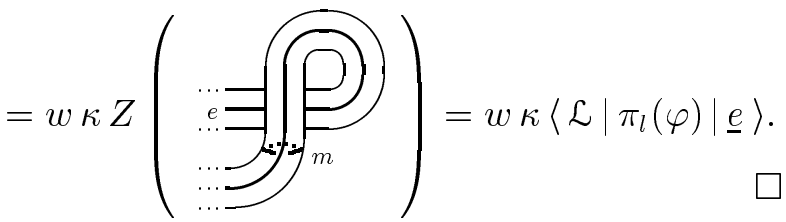

This relation also explains the local meaning of $\mathcal{L}$ and $\mathcal{L}^{\prime}$. The normalization of $(4-4)$ is consistent with

$$
\left\langle\mathcal{L}\left|\pi_{l}\left(A_{1}^{n_{1}} \cdots A_{N}^{n_{N}}\right)\right| \underline{0}, \underline{0}\right\rangle=\langle\mathcal{L} \mid \underline{0}, \underline{0}\rangle=w^{-2}
$$

and

$$
\begin{aligned}
\left\langle\underline{e}\left|\pi_{l}\left(S_{1} \cdots S_{N}\right)\right| \underline{0}\right\rangle & =S_{e_{1} 0} \cdots S_{e_{N} 0} \\
& =w^{-N} w_{e_{1}}^{2} \cdots w_{e_{N}}^{2} .
\end{aligned}
$$

Invariance under Kirby moves obviously does not determine uniquely the phase factor in (4-4). In Section 5 we present for special cases an experimentally obtained choice of the phase factor exp $i \psi$ such that $Z_{l}(M, r)$ fits asymptotically for $r \rightarrow \infty$ with the asymptotic expansion of Witten's ChernSimons functional integral $Z_{\mathrm{CS}}(M, k)$ for

$$
k=r+2 \rightarrow \infty .
$$

This choice of the phase does not agree with that of (4-4). Formula (4-4) yields up to a factor $w$ the Reshetikhin-Turaev invariant of three-manifolds [Reshetikhin and Turaev 1991] in the slightly modified version of Kirby and Melvin [1991] (see also [Wenzl 1993]):

$$
Z_{l}(M, r)=w^{-1} \tau_{r}(M) .
$$

The matrix element

$$
\left\langle\mathcal{L}\left|A_{1}^{n_{1}} \cdots A_{N}^{n_{N}}\right| \underline{e}\right\rangle
$$

in $(4-4)$ is the invariant of the link $\mathcal{L}$ with framings $n_{1}, \ldots, n_{N}$ of the components as given by formulas (2-7) and (2-8) for left-handed lines with colours $\underline{e}=\left(e_{1}, \ldots, e_{N}\right)$. In particular for a single unknotted circle $(N=1)$ and framing $n= \pm 1,(4-4)$ together with (2-8) and (3-7) yields the following value for the invariant of $S^{3}$ (see also Figure 7):

$$
\begin{aligned}
Z_{l}\left(S^{3}, r\right) & =\kappa^{\mp 1} \sum_{e}\left\langle\mathcal{L}\left|\pi_{l}\left(A^{ \pm 1}\right)\right| e\right\rangle w_{e}^{2} \\
& =\kappa^{\mp 1} w^{-2} \sum_{e} w_{e}^{4} q_{e}^{ \pm 2}=w^{-1} .
\end{aligned}
$$

This means that $Z_{l}(M, r)$ is invariant under Kirby moves in this case.

We now return to Equation (4-3) and restrict attention to the case where $\Sigma$ is a (possibly knotted) single torus $S^{1} \times S^{1}$ forming a knot $\mathcal{K}$ of one component. Then

$$
Z_{l}(M, r)=e^{i \psi} w \sum_{e}\langle\mathcal{K} \mid e\rangle\left\langle e\left|\pi_{l}(\varphi)\right| 0\right\rangle,
$$

which is the desired formula. The general case for the map $\varphi$ belongs to a $(-n / m)$-surgery. We distinguish two cases, always with $m \neq 0$ :

(i) $|n / m|>1$. Then we write $n / m$ as a finite continued fraction expansion

$$
\frac{n}{m}=m_{t}-\frac{1}{m_{t-1}-\frac{1}{\cdots-\frac{1}{m_{1}}}},
$$

for $1 \leq t<\infty$ with integers $m_{1}, \ldots, m_{t}$ each $\geq 2$. The mapping class group element is then of the form

$$
\varphi^{-n / m}=A^{m_{t}} S A^{m_{t-1}} S \cdots A^{m_{1}} S,
$$


and the matrix element in (4-7) is given by the matrix product

$$
\left\langle e\left|\pi_{l}\left(\varphi^{-n / m}\right)\right| 0\right\rangle=\left(A^{m_{t}} S \cdots A^{m_{1}} S\right)_{e 0},
$$

where $A$ is the diagonal matrix of $(3-16)$ with diagonal elements $q_{a}^{2}$ and $S$ is the Verlinde matrix $(3-24)$.

(ii) $|n / m|<1$. This time we write

$$
\frac{m}{n}=m_{t}-\frac{1}{m_{t-1}-\frac{1}{\cdots-\frac{1}{m_{1}}}},
$$

again with the $m_{i} \geq 2$. The mapping class group element is then of the form

$$
\varphi^{-n / m}=S A^{m_{t}} S A^{m_{t-1}} S \cdots A^{m_{1}} S,
$$

and the matrix element in (4-7) is given by the matrix product

$$
\left\langle e\left|\pi_{l}\left(\varphi^{-n / m}\right)\right| 0\right\rangle=\left(S A^{m_{t}} S \cdots A^{m_{1}} S\right)_{e 0} .
$$

We obtain, in particular,

$$
\begin{aligned}
\left\langle e\left|\pi_{l}\left(\varphi^{-n / 1}\right)\right| 0\right\rangle & =q_{e}^{2 n} S_{e 0}=w^{-1} q_{e}^{2 n} w_{e}^{2} \\
\left\langle e\left|\pi_{l}\left(\varphi^{-1 / m}\right)\right| 0\right\rangle & =\sum_{e^{\prime}} S_{e e^{\prime}} q_{e^{\prime}}^{-2 m} S_{e^{\prime} 0}
\end{aligned}
$$

What remains is to calculate the coefficients $\langle\mathcal{K} \mid e\rangle$ depending on the way $\mathcal{K}$ is embedded in $S^{3}$. They are invariants of knots.

We now assume in addition that $\mathcal{K}$ is a figureeight knot.

Lemma 4.2. If $\mathcal{K}$ is the figure-eight knot, then

$$
\langle\mathcal{K} \mid e\rangle=w^{-2} \sum_{b, c} w_{b}^{2} w_{c}^{2} q_{b}^{2} q_{c}^{-2}\left|\begin{array}{lll}
e & e & b \\
e & e & c
\end{array}\right| .
$$

Proof. We use Theorem 2.11 to write the invariant of the knot embedded in $S^{3}$ as an invariant of a planar graph

$$
\begin{aligned}
& \langle\mathcal{K} \mid e\rangle=Z(e \\
& =w^{-2} Z(e \stackrel{\Omega}{g})
\end{aligned}
$$

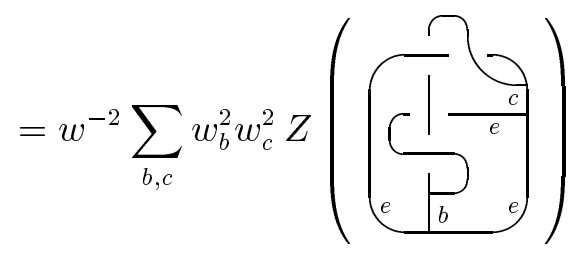

$$
\begin{aligned}
& =w^{-2} \sum_{b, c} w_{b}^{2} w_{c}^{2} q_{b}^{2} q_{c}^{-2} Z\left(\left(\begin{array}{c|c}
e & c \\
e & e \\
\cline { 2 - 2 } & e
\end{array}\right) .\right.
\end{aligned}
$$

The completeness and Racah relations (B-1) have been used. By Equation (2-4) we obtain Equation (4-12).

Corollary 4.3. For the figure-eight knot and $a(-n / m)$ surgery the resulting manifold $M_{8}(-n / m)$ has a Turaev-Viro state sum $Z\left(M_{8}(-n / m)\right)$ equal to

$$
\left|Z_{l}\left(M_{8}(-n / m)\right)\right|^{2}
$$

with

$$
\begin{aligned}
& Z_{l}\left(M_{8}(-n / m), r\right)=e^{i \psi} w^{-1} \\
& \times \sum_{b, c, e} w_{b}^{2} w_{c}^{2} q_{b}^{2} q_{c}^{-2}\left|\begin{array}{lll}
e & e & b \\
e & e & c
\end{array}\right|\left\langle e\left|\pi_{l}\left(\varphi^{-n / m}\right)\right| 0\right\rangle .
\end{aligned}
$$

Example 4.4. We may write Equation (4-7) also as $Z_{l}(M, r)=e^{i \psi} w^{-1} \sum_{e}\left\langle\mathcal{K}\left|\pi_{l}(\hat{\varphi})\right| e\right\rangle\left\langle e\left|\pi_{l}(S)\right| 0\right\rangle$

with $\varphi=\hat{\varphi} S$. For a $(-2 / 1)$-surgery at a figureeight knot we have 


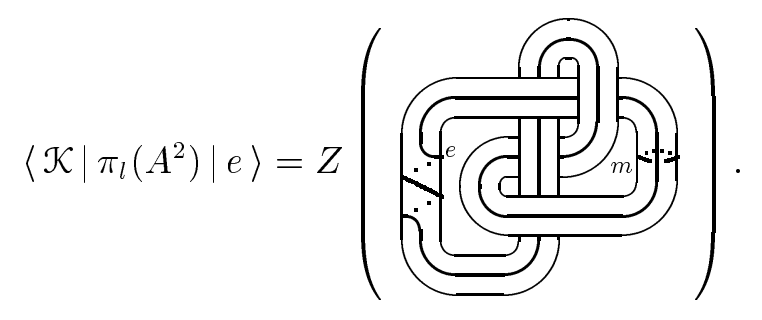

The meridian $m$ undercrosses the line with colour $e$ such that it is left-handed. For a $(-1 / 2)$-surgery at a figure-eight knot we have

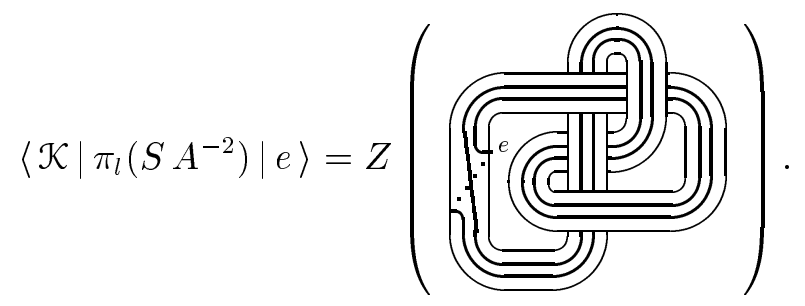

For simplicity we have not drawn the image of the meridian.

\section{NUMERICAL RESULTS}

We have analyzed numerically the partition function $Z\left(M_{8}(-n / m), r\right)$ given by Equations (4-10)(4-13) for several cases. For this we used the explicit formula for the $6 j$-symbol taken from [Kirillov and Reshetikhin 1989], with the identification

$$
\left|\begin{array}{lll}
a & b & e \\
d & c & f
\end{array}\right|=i^{-2(a+b+c+d+e+f)}\left\{\begin{array}{lll}
a & b & e \\
d & c & f
\end{array}\right\}^{\mathrm{RW}}
$$

where the right-hand side is the quantity defined in [Kirillov and Reshetikhin 1989, Theorem 5.1] as follows:

$$
\begin{aligned}
& \left\{\begin{array}{lll}
a & b & e \\
d & c & f
\end{array}\right\}^{\mathrm{RW}}=\triangle(a b e) \triangle(a c f) \triangle(c d e) \triangle(d b f) \\
& \quad \times \sum_{z}(-1)^{z}[z+1]_{q} !\left([z-a-b-e]_{q} ![z-a-c-f]_{q} !\right. \\
& \quad \times[z-b-d-f]_{q} ![z-d-c-e]_{q} ![a+b+c+d-z]_{q} ! \\
& \left.\quad \times[a+d+e+f-z]_{q} ![b+c+e+f-z]_{q} !\right)^{-1}
\end{aligned}
$$

with the notation

$$
\triangle(a b c)=\left(\frac{[-a+b+c]_{q} ![a-b+c]_{q} ![a+b-c]_{q} !}{[a+b+c+1]_{q} !}\right)^{\frac{1}{2}}
$$

as in [Karowski and Schrader 1993, (3.6)],

$$
[n]_{q} !=\left(\frac{q^{1}-q^{-1}}{q-q^{-1}}\right)\left(\frac{q^{2}-q^{-2}}{q-q^{-1}}\right) \cdots\left(\frac{q^{n}-q^{-n}}{q-q^{-1}}\right),
$$

and the sum in (5-2) taken over all nonnegative integers $z$ with nonnegative arguments in the square brackets.

(Note that the $q^{1 / 2}$ of [Kirillov and Reshetikhin 1989] is our $q$ and the $q_{0}$ of [Turaev and Viro 1992]).

The numerical calculations were performed for various manifolds $M_{8}(-n / m)$ and values of $r$ in the range $4 \leq r \leq 403$. We used a Fortran program with double precision. Note that due to Equations (4-12) and (5-1)-(5-3) the numerical calculation is essentially a four-loop calculation, so $Z_{l}\left(M_{8}(-n / m), r\right)$ is a sum of $O\left(r^{4}\right)$ contributions with a corresponding computing time behaviour. This is why for values of $r$ above $r \approx 440$ the results tended to become unreliable in double precision. It is possible to extend the range of $r$ up to $\sim 500$ by working with G-precision. Here, however, we shall only report on results in the range given above. The absolute values of $Z_{l}\left(M_{8}(-n / m), r\right)$ turn out to be all of order one, in contrast to the case of lens spaces (see [Freed and Gompf 1991; Jeffrey 1992], for example).

Example 5.1. Figure 12, left, plots $Z_{l}\left(M_{8}(-6 / 1), r\right)$ for even $r=4, \ldots, 403$ as dots in the complex plane. The phase in Equation (4-13) is taken as $\psi=\pi r$; see Equation (5-8) below. It turns out that for odd $r$ these invariants are zero (see below and Appendices $\mathrm{C}$ and D). Note that $M_{8}(-6 / 1)$ is a hyperbolic manifold.

Example 5.2. Figure 12, right, plots $Z_{l}\left(M_{8}(-1 / 6), r\right)$ for $r=4, \ldots, 403$. The phase in Equation (4-13) for $m=1$ is taken as $\psi=0$; see Equation (5-10) below.

The main interest of our analysis was to see if the semiclassical analysis $r \rightarrow \infty$ could be correct. 

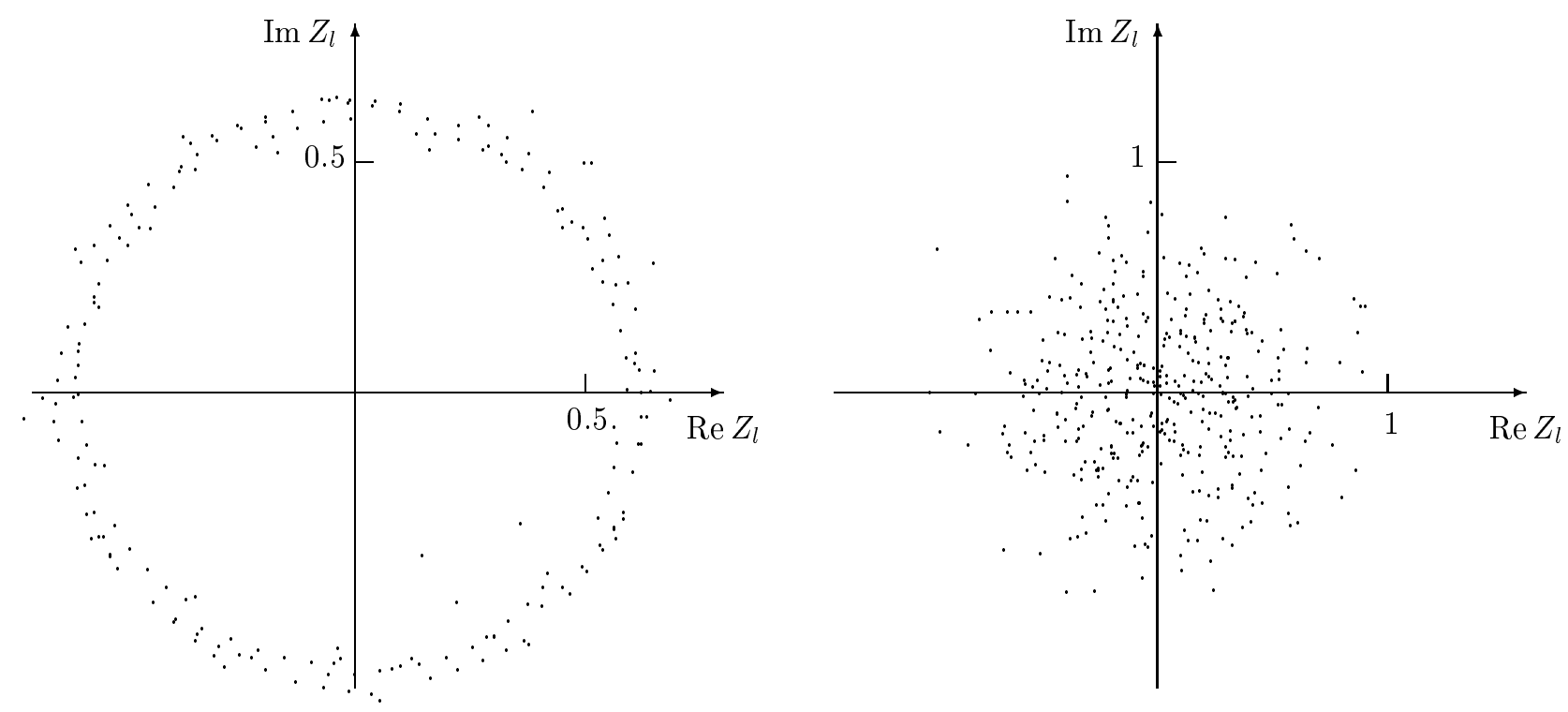

FIGURE 12. Left: The values of $Z_{l}\left(M_{8}(-6 / 1), r\right)$, for even $4 \leq r \leq 403$. Right: The values of $Z_{l}\left(M_{8}(-1 / 6), r\right)$, for $4 \leq r \leq 403$.

Recall that by Equation (1-1) (see also (4-8) and $(4-13))$ the values of $Z_{l}\left(M_{8}(-n / m), r\right)$ and $Z_{\mathrm{CS}}\left(M_{8}(-n / m), k=r-2\right)$ should agree up to a phase. Now the semiclassical conjecture [Witten 1989; Freed and Gompf 1991; Rozansky 1995] is that for large $r$ we have

$$
\begin{aligned}
& Z_{\mathrm{CS}}(M, k=r-2) \\
& \quad \cong \frac{1}{2} \sum_{A \text { flat }} C_{A} r^{-\frac{1}{2} \operatorname{dim} H^{0}(A)} \cdot e^{2 \pi i r S_{\mathrm{CS}}(A)},
\end{aligned}
$$

if all flat connections are isolated (modulo gauge transformations), so that in particular we then have $\operatorname{dim} H^{1}(A)=0$. This is indeed the case for all examples we have considered. Therefore the leading contributions in Equation (5-4) arise from the irreducible flat bundles, where $H^{0}(A)=0$. Since the structure group is $\mathrm{SU}(2)$ and since we consider the adjoint representation, $\operatorname{dim} H^{0}(A)$ can only take the values 0 (in the irreducible case), 1 (reducible to $U(1)$ ) or 3 (in the trivial case with $S_{\mathrm{CS}}(A)=0$ ). Finally, the absolute value of $C_{A}^{2}$ is conjectured to be, say for the irreducible connections, equal to the Reidemeister-Ray-Singer torsion, whereas the phase is related to the spectral flow [Witten 1989; Freed and Gompf 1991; Rozansky 1995].
To see if (5-4) holds we performed a Fourier transform

$$
\begin{aligned}
\tilde{Z}_{l}\left(M_{8}(\right. & -n / m), t) \\
& =\frac{1}{400} \sum_{r=4}^{403} Z_{l}\left(M_{8}(-n / m), r\right) e^{-2 \pi i t r},
\end{aligned}
$$

where $0 \leq t \leq 1$. This function should show prominent maxima at values of $t$ that correspond to $S_{\mathrm{CS}}(A)$ for flat $A$. In principle the absolute values of the $C_{A}$ 's may be determined from $\left|\tilde{Z}_{l}(M, t)\right|$ at the maxima unless there are two or more different flat connections with the same Chern-Simons action and the same $\operatorname{dim} H^{0}(A)$. This is for example the case for $M_{8}(-n / 1)$ with $n=8,16,20$ (see Appendix C).

Example 5.3. Figure 13, left, plots the absolute value of the Fourier transform $\tilde{Z}_{l}\left(M_{8}(-6 / 1), t\right)$ where the phase in Equation (4-13) for $m=1$ has be taken as $\psi=\pi r$; see Equation (5-8) below. The function has period $t=\frac{1}{2}$ since $Z_{l}\left(M_{8}(-6 / 1), r\right)$ vanishes for odd $r$. We interpret the big peaks as corresponding to the leading contributions of the asymptotic expansion $(5-4)$ with $\operatorname{dim} H^{0}(A)=0$ and the small ones to $\operatorname{dim} H^{0}(A)=1$. 

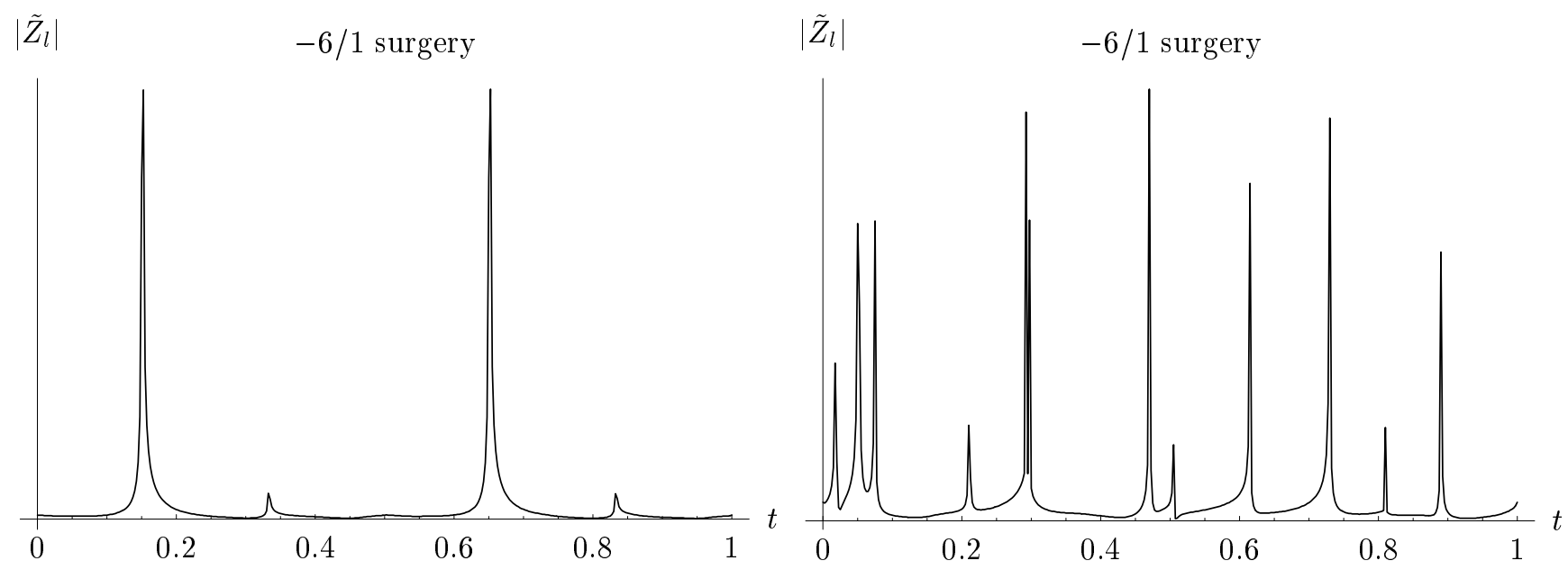

FIGURE 13. Left: The Fourier transform $\left|\tilde{Z}_{l}\left(M_{8}(-6 / 1), t\right)\right|$. Right: $\left|\tilde{Z}_{l}\left(M_{8}(-1 / 6), t\right)\right|$.

Example 5.4. Figure 13, right, plots the absolute value of $\tilde{Z}_{l}\left(M_{8}(-1 / 6), t\right)$ where the phase in Equation (4-13) has been taken as $\psi=0$; see Equation $(5-10)$ below.

To locate the maxima of $\left|\tilde{Z}_{l}\left(M_{8}(-n / m), t\right)\right|$, we also employed the following procedure. We considered the set of points

$$
Z_{l}\left(M_{8}(-n / m), r, t\right)=Z_{l}\left(M_{8}(-n / m), r\right) e^{-2 \pi i t r} .
$$

For $t$ at a maximum these members typically form a symmetric pattern. Figure 14 provides two examples at the maxima $t=0.15127$ and $t=0.33333$ for the manifold $M_{8}(-6 / 1)$ (compare Figure 13 and Tables 1 and 2). Figure 12, left, corresponds to the choice $t=0$.

In all the examples considered, this procedure allowed for an evaluation of the maxima up to six digits. Since $Z_{\mathrm{CS}}$ and $Z_{l}$ might differ by a phase factor, the differences in the maxima should correspond to the differences in the Chern-Simons invariants of flat bundles.
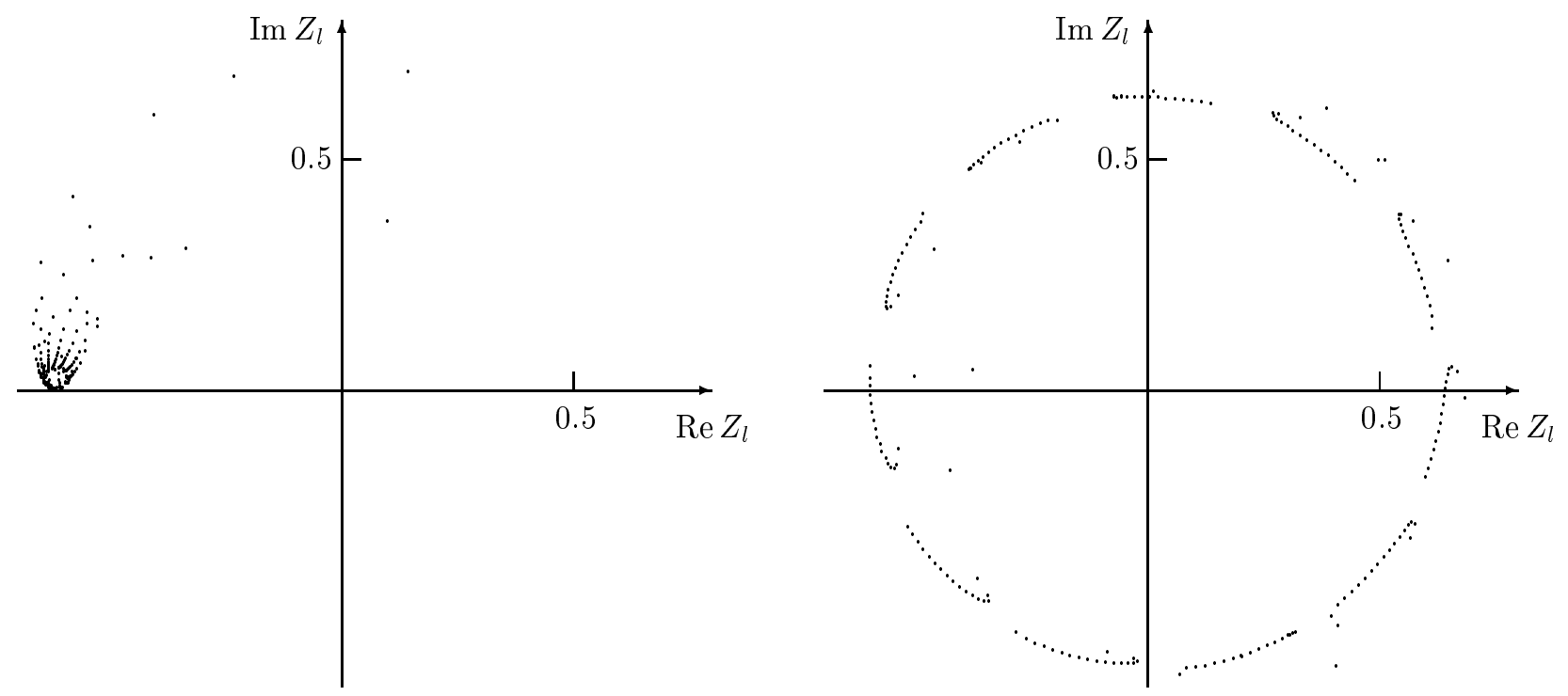

FIGURE 14. The values of $Z_{l}\left(M_{8}(-6 / 1), r\right) e^{-2 \pi i t r}$ for $t=0.15127$ (left) $t=0.33333$ (right). 
Kirk and Klassen [1990] describe explicitly how to obtain all reducible and irreducible flat connections up to gauge equivalence of $M_{8}(n / m)$ and give precise formulae for computing the corresponding Chern-Simons invariants. For the convenience of the reader we briefly recall these results in Appendix $\mathrm{C}$, where we also derive some consequences from these formulae. The set of Chern-Simons invariants of $M_{8}(n / m)$ corresponding to reducible flat connections is given by

$$
\left\{-\frac{t^{2} p}{n} \mid t=0,1, \ldots,\left\lfloor\frac{1}{2} n\right\rfloor\right\}
$$

where $p$ is an integer such that $p \cdot m \equiv-1 \bmod n$ (see Appendix C). In our numerical analysis the reducible flat connections show up as small peaks (see Example 5.3, Figure 13, left, and Table 1). This is obviously due to the fact that these contributions in formula (5-4) go like $r^{-1 / 2}$. Comparing these values (5-6) with the values obtained from our numerical calculations starting from (4-13), we can determine (at least asymptotically for $r \rightarrow \infty$ ) the phase $\psi$ in (4-3) and (4-13), which is undetermined from the Turaev-Viro invariants. For all examples of three-manifolds $M_{8}(-n / m)$ we have considered the phase is consistent with

$$
\psi(n, m, r)=\frac{\pi}{2} K(n, m) r,
$$

with $K(n, m) \in \mathbb{Z}_{4}$, at least asymptotically for large $r$.

In Table 1 we list for some examples the number $K(n, m)$ and the values of $S_{\mathrm{CS}}$ obtained by our numerical method compared with the values obtained by the method of [Kirk and Klassen 1990].

Kirk and Klassen [1990] provide for the examples $(n / m)=(-3 / 1),(-1 / 1),(-1 / 2)$, and $(-1 / 3)$ a list of all irreducible representations of $\pi_{1} M_{8}(n / m)$ and the corresponding Chern-Simons invariants. With the techniques presented in Appendix $\mathrm{C}$ we have extended their list by more examples to compare the values of $S_{\mathrm{CS}}(A)$ predicted by the semiclassical approximation formula (5-1) with the actual values given by Equations $(\mathrm{C}-3)$ and $(\mathrm{C}-4)$.

\begin{tabular}{|ccccc|}
\hline surgery & \# red. reps & $K$ & $S_{\mathrm{CS}}(4-13)$ & $S_{\mathrm{CS}}(\mathrm{K}-\mathrm{K})$ \\
\hline$-3 / 1$ & 1 & 3 & 0.66681 & $2 / 3$ \\
\hline$-4 / 1$ & 1 & 0 & 0.75000 & $3 / 4$ \\
\hline$-5 / 1$ & 2 & 1 & 0.19997 & $1 / 5$ \\
& & & 0.79999 & $4 / 5$ \\
\hline$-6 / 1$ & \multirow{2}{*}{3} & 2 & 0.3333 & $1 / 3$ \\
& & & 0.5012 & $1 / 2$ \\
& & & 0.83333 & $5 / 6$ \\
\hline
\end{tabular}

TABLE 1. Values of $S_{\mathrm{CS}}(A)$ as obtained from (4-13) and from [Kirk and Klassen 1990], for some examples of reducible representations for $(-n / 1)$ surgery.

We used Mathematica to find the solutions of $(\mathrm{C}-2)$ and to calculate the corresponding values of Equations $(\mathrm{C}-3)$ and $(\mathrm{C}-4)$.

Some of these results are listed in Tables 2, 3, and 4 . They too show excellent agreement between the two methods. The following comments also take into account other examples not listed in these tables.

Example 5.5. For $(-n / 1)$-surgery (see Table 2$)$ the phase factor in Equation (4-13) turns out to be consistent with the choice

$$
e^{i \psi}=e^{i \pi n r / 2}, \quad \text { i.e., } \quad K(n, 1)=n,
$$

at least asymptotically for large $r$. Note that this phase factor is an invariant of the three-manifold $M_{8}(-n / 1)$, because the modified ReshetikhinTuraev invariant $\tau_{r}(M)$ of [Kirby and Melvin 1991] (see (4-5)) takes the following values for $r=3$ :

$$
\tau_{3}\left(M_{8}(-n / 1)\right)=\kappa \frac{1}{\sqrt{2}}\left(1+e^{-i \pi n / 2}\right) .
$$

This result is obtained as follows. For $r=3$ in (4-13) only $b=c=0$ and $e=0,1 / 2$ contribute. Therefore with Equation (4-10) we have

$$
Z_{l}\left(M_{8}(-n / 1), 3\right)=e^{i \psi} w^{-2} \sum_{e=0}^{1 / 2} w_{e}^{2} q_{e}^{2 n}\left|\begin{array}{lll}
e & e & 0 \\
e & e & 0
\end{array}\right|
$$




\begin{tabular}{|c|c|c|c|c|}
\hline surgery & \# irr. reps & $K$ & $S_{\mathrm{CS}}(4-13)$ & $S_{\mathrm{CS}}(\mathrm{K}-\mathrm{K})$ \\
\hline$-0 / 1$ & 0 & 3 & $\begin{array}{l}0.00000 \\
0.20000 \\
0.80000\end{array}$ & $\begin{array}{l}0.0 \\
0.2 \\
0.8\end{array}$ \\
\hline$-1 / 1$ & 2 & 1 & $\begin{array}{l}0.27978 \\
0.85116\end{array}$ & $\begin{array}{l}0.279762 \\
0.8511905\end{array}$ \\
\hline$-2 / 1$ & 2 & 2 & $\begin{array}{l}0.387512 \\
0.88750\end{array}$ & $\begin{array}{l}0.3875 \\
0.8875\end{array}$ \\
\hline$-3 / 1$ & 2 & 3 & $\begin{array}{l}0.479178 \\
0.91667\end{array}$ & $\begin{array}{l}0.479166 \\
0.91666\end{array}$ \\
\hline$-4 / 1$ & 2 & 0 & $\begin{array}{l}0.55019 \\
0.950009\end{array}$ & $\begin{array}{l}0.55 \\
0.95\end{array}$ \\
\hline$-5 / 1$ & 2 & 1 & $\begin{array}{l}0.054478 \\
0.605920\end{array}$ & $\begin{array}{l}0.054458 \\
0.6059135\end{array}$ \\
\hline$-6 / 1$ & 2 & 2 & $\begin{array}{l}0.151275 \\
0.651283\end{array}$ & $\begin{array}{l}0.151265 \\
0.651265\end{array}$ \\
\hline$-8 / 1$ & 4 & 0 & $\begin{array}{l}0.29997 \\
0.70003 \\
0.721091 \\
0.721091\end{array}$ & $\begin{array}{l}0.3 \\
0.7 \\
0.721304 \\
0.721304\end{array}$ \\
\hline$-12 / 1$ & 4 & 0 & $\begin{array}{l}0.050089 \\
0.450186 \\
0.489647 \\
0.489647\end{array}$ & $\begin{array}{l}0.05 \\
0.45 \\
0.4896907 \\
0.4896907\end{array}$ \\
\hline$-14 / 1$ & 6 & 2 & $\begin{array}{l}0.048320 \\
0.055263 \\
0.161499 \\
0.548319 \\
0.555265 \\
0.661497\end{array}$ & $\begin{array}{l}0.0485379 \\
0.054853 \\
0.1614793 \\
0.5485379 \\
0.554853 \\
0.6614793\end{array}$ \\
\hline$-16 / 1$ & 4 & 0 & $\begin{array}{l}0.200064 \\
0.251354 \\
0.251354 \\
0.799979\end{array}$ & $\begin{array}{l}0.2 \\
0.251307 \\
0.251307 \\
0.8\end{array}$ \\
\hline$-18 / 1$ & 6 & 2 & $\begin{array}{l}0.306522 \\
0.325624 \\
0.413818 \\
0.806520 \\
0.825627 \\
0.913822\end{array}$ & $\begin{array}{l}0.3064713 \\
0.3255013 \\
0.413881 \\
0.8064713 \\
0.8255013 \\
0.913881\end{array}$ \\
\hline
\end{tabular}

TABLE 2. Values of $S_{\mathrm{CS}}(A)$ for some examples of $(-n / 1)$-surgery for irreducible representations. See Table 1 for the meaning of the last two columns.
Now Equations (2-2), (2-5), and (2-9) yield $w_{0}^{2}=1$, $w_{1 / 2}^{2}=-1, q_{0}=1, q_{1 / 2}=-q^{3 / 2}=e^{-i \pi / 2}, w^{2}=2$, and

$$
\left|\begin{array}{lll}
0 & 0 & 0 \\
0 & 0 & 0
\end{array}\right|=1, \quad\left|\begin{array}{ccc}
\frac{1}{2} & \frac{1}{2} & 0 \\
\frac{1}{2} & \frac{1}{2} & 0
\end{array}\right|=-1
$$

(see [Turaev and Viro 1992], for example), giving $Z_{l}\left(M_{8}(-n / 1), 3\right)=e^{i \psi} \frac{1}{2}\left(1+e^{-i \pi n / 2}\right)$. In particular for $n=4 n^{\prime}+2$ this vanishes. As already stated, the big peaks in the function $\tilde{Z}_{l}(M, t)$ (compare Figure 13 for $n=6$ ) correspond to the leading contributions of the asymptotic expansion (5-4) with $\operatorname{dim} H^{0}(A)=0$ and the small ones to $\operatorname{dim} H^{0}(A)=1$.

(a) For $m=1$ and $n>4$, in which case the space $M_{8}(-n / m)$ is hyperbolic, there is at least one flat $\mathrm{SU}(2)$-connection $A$ with vanishing cohomology. For increasing $n$ there is a proliferation in the number of such flat connections (modulo gauge equivalence). See the remarks after Equation (C-2) in Appendix C.

(b) The numerical calculations confirm the analytic results of Appendices $\mathrm{C}$ and $\mathrm{D}$ to the effect that $Z_{l}\left(M_{8}(-n / 1), r\right)=0$ for all odd $r$ in case $n \equiv 2$ $\bmod 4$. The case $n=6$ is depicted in Figure 13, left, showing that $\tilde{Z}_{l}\left(M_{8}(-6 / 1), t\right)$ is indeed periodic in $t$ with period $\frac{1}{2}$.

(c) For the exceptional case of $M_{8}(0 / 1)$, which for the case of an unknotted torus would correspond to $S^{2} \times S^{1}$ we find the closed formula (guessed by our numerical results)

$$
Z_{l}\left(M_{8}(0 / 1), r\right)=\frac{\cos 2 \pi / 5-\cos 2 \pi r / 5}{2 \sin \pi / 5 \sin 2 \pi / 5} .
$$

In this case the semiclassical approximation is exact. This is analogous to the situation for lens spaces [Jeffrey 1992]. This result also agrees with the analytic result of Kirk and Klassen [1990] according to which there are two ChernSimons invariants with values $\pm \frac{1}{5}$ (and vanishing cohomology).

(d) For the case $n=1, m=1$, corresponding to the Seifert homology sphere $-\Sigma(2,3,7)$, we obtain two maxima for $\left|\tilde{Z}_{l}\right|$ located near

$$
1-\frac{25}{4 \cdot 42} \quad \text { and } \quad 1-\frac{121}{4 \cdot 42}
$$


This is compatible with the result of Kirk and Klassen giving two flat connections $A_{1}$ and $A_{2}$ with

$$
S_{\mathrm{CS}}\left(A_{1}\right)=-\frac{11^{2}}{4 \cdot 42} \text { and } S_{\mathrm{CS}}\left(A_{2}\right)=-\frac{5^{2}}{4 \cdot 42} .
$$

(e) For $n=3, m=1$, corresponding to a Seifert fibered space over the two-sphere with Seifert invariants $(3,-2),(3,1),(4,1)$, Kirk and Klassen predict two values

$$
S_{\mathrm{CS}}\left(A_{1}\right)=-\frac{2^{2}}{4 \cdot 12} \quad \text { and } \quad S_{\mathrm{CS}}\left(A_{2}\right)=-\frac{5^{2}}{4 \cdot 12} .
$$

We have found two strong maxima of $\tilde{Z}_{l}$ at $t_{1}=$ $1-5^{2} /(4 \cdot 12)$ and $t_{2}=1-2^{2} /(4 \cdot 12)$ and a small maximum at $t_{3}=2 / 3$ if we choose $K(3,1)=3$ in Equation (5-7); see (5-8).

(f) If $n=4 k$ with $k>1$, there are at least two irreducible representations of $\pi_{1}\left(M_{8}(-n / 1)\right)$ with the same Chern-Simons actions (for a precise statement see Proposition C.1 below). We have taken account of this fact in Table 2, although of course the analysis of Equation (4-12) does not exhibit this. Table 2 provides examples of all statements of Proposition C.1.

Example 5.6. For $(-1 / m)$-surgery (see Table 3 ) the phase factor in (4-13) turns out to be consistent with the choice

$$
e^{i \psi}=1, \quad \text { i.e., } \quad K(1, m)=0
$$

(again at least asymptotically for large $r$ ), and the numerical results are in agreement with the observation made above that there are $2 m$ irreducible flat connections (modulo gauge equivalence). Since the $M_{8}(-1 / m)$ are homology spheres there are no nontrivial reducible representations.

Example 5.7. Figure 15 plots the absolute value of the Fourier transforms

$$
\tilde{Z}_{l}\left(M_{8}(-3 / 2), t\right) \text { and } \tilde{Z}_{l}\left(M_{8}(-2 / 3), t\right) .
$$

In each case the maxima are at the same values as

\begin{tabular}{|c|c|c|c|}
\hline surgery & \# irr. reps & $S_{\mathrm{CS}}(4-13)$ & $S_{\mathrm{CS}}(\mathrm{K}-\mathrm{K})$ \\
\hline \multirow[t]{4}{*}{$-1 / 2$} & 4 & 0.053927 & 0.053933 \\
\hline & & 0.235109 & 0.23516 \\
\hline & & 0.514125 & 0.514125 \\
\hline & & 0.828180 & 0.828117 \\
\hline \multirow[t]{12}{*}{$-1 / 6$} & 12 & 0.018324 & 0.018451 \\
\hline & & 0.051140 & 0.051163 \\
\hline & & 0.074568 & 0.07461 \\
\hline & & 0.211492 & 0.21082 \\
\hline & & 0.291498 & 0.292507 \\
\hline & & 0.298875 & 0.297362 \\
\hline & & 0.470291 & 0.470357 \\
\hline & & 0.504663 & 0.504638 \\
\hline & & 0.614698 & 0.614739 \\
\hline & & 0.729624 & 0.729587 \\
\hline & & 0.810647 & 0.810042 \\
\hline & & 0.890340 & 0.890394 \\
\hline \multirow[t]{16}{*}{$-1 / 8$} & 16 & 0.003120 & 0.00348 \\
\hline & & 0.086381 & 0.08637 \\
\hline & & & 0.17133 \\
\hline & & 0.172185 & 0.172535 \\
\hline & & 0.207460 & 0.20804 \\
\hline & & 0.272334 & 0.272329 \\
\hline & & 0.400936 & 0.40089 \\
\hline & & 0.415939 & 0.415986 \\
\hline & & 0.514109 & 0.51386 \\
\hline & & 0.555343 & 0.55579 \\
\hline & & 0.593689 & 0.593654 \\
\hline & & 0.720535 & 0.7205257 \\
\hline & & 0.808226 & 0.807600 \\
\hline & & 0.850603 & 0.85053 \\
\hline & & 0.867921 & 0.868405 \\
\hline & & 0.989921 & 0.990035 \\
\hline
\end{tabular}
calculated using the methods of Kirk and Klassen [1990, p. 363], that is, $Z_{l}$ seems to agree with $Z_{\mathrm{CS}}$ in these cases, which means that $K(2,3)=K(3,2)=0$.
TABLE 3. Values of $S_{\mathrm{CS}}$ for examples of $(-1 / \mathrm{m})$ surgery. For $-1 / 8$ one spike at $S_{\mathrm{CS}}=0.17133$ is not discernible, since it is too close to the one at $S_{\mathrm{CS}}=0.172535$.

Example 5.8. We analyzed numerically the phase factor $e^{i \psi}$ for manifolds $M_{8}(-n / m)$ with $n>0$, $m>0, n / m>1$, and values of $m$ from 2 to 15. We looked at about 400 manifolds in total. Our numerical results indicate the following rules: Asymptotically for large $r$ the phase $\psi$ is again of the form $(5-7)$. In particular $K(n, m)$ is fixed only $\bmod 2$ if $Z_{l}\left(M_{8}(-n / m), r\right)=0$ for $r$ odd. Also 

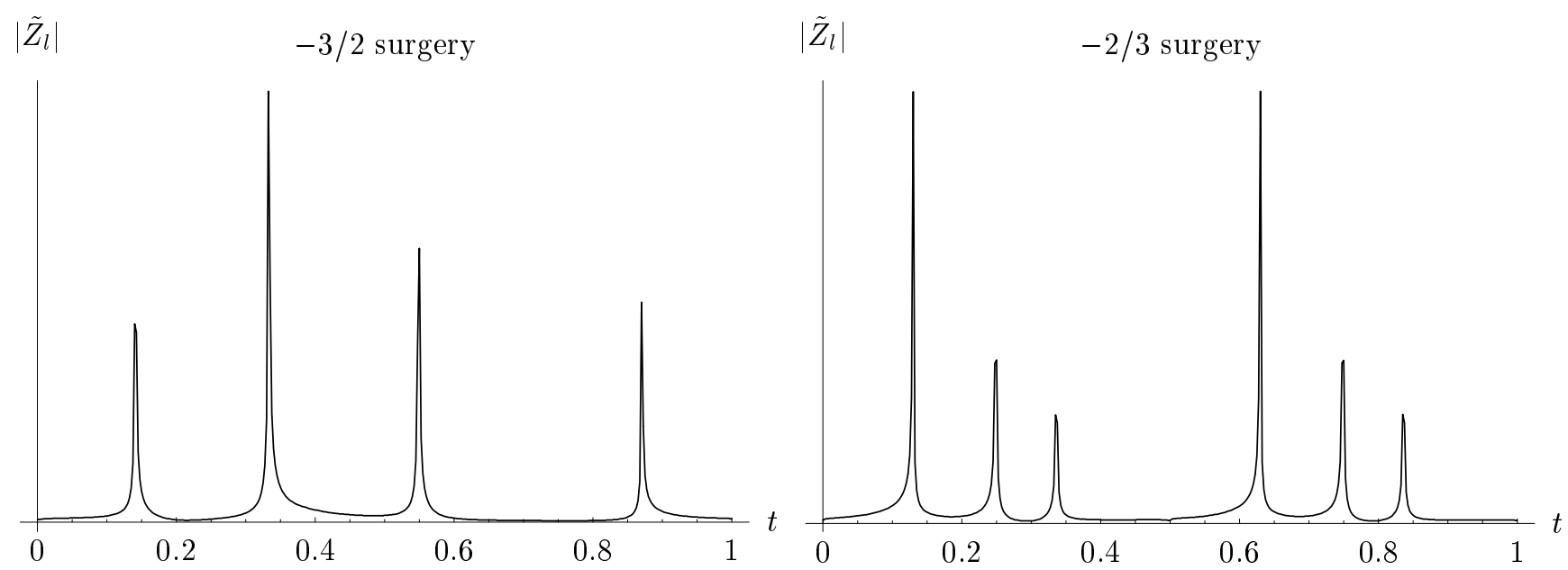

FIGURE 15. Left: The Fourier transform $\left|\tilde{Z}_{l}\left(M_{8}(-3 / 2), t\right)\right|$. Right: $\left|\tilde{Z}_{l}\left(M_{8}(-2 / 3), t\right)\right|$.

\begin{tabular}{|ccccc|}
\hline surgery & \# irr. reps & $K$ & $S_{\mathrm{CS}}(4-13)$ & $S_{\mathrm{CS}}(\mathrm{K}-\mathrm{K})$ \\
\hline$-3 / 2$ & 4 & 0 & 0.141068 & 0.141138 \\
& & & 0.333369 & 0.333333 \\
& & & 0.548895 & 0.548930 \\
& & & 0.870718 & 0.870641 \\
\hline$-2 / 3$ & 6 & 0 & 0.129545 & 0.129563 \\
& & & 0.248862 & 0.248874 \\
& & & 0.336324 & 0.336288 \\
& & & 0.629556 & 0.629563 \\
& & & 0.748850 & 0.748874 \\
& & & 0.836311 & 0.836288 \\
\hline
\end{tabular}

TABLE 4. Values of $S_{\mathrm{CS}}$ for $(-3 / 2)$ and $(-2 / 3)$ surgery.

$K(n, m)$ is quasiperiodic in $n$ with quasiperiod $\pm m$; that is,

$$
K(n+m, m) \equiv K(n, m) \pm m \bmod 4,
$$

with a minus sign if $m \equiv 1 \bmod 4$ and a plus sign otherwise. We have not been able to find a general closed form for $K(n, m)$ as a function of $n$ and $m$. In Table 5 we have listed the values of $K$ for $m=1, \ldots, 12$ and some low values of $n>m$. For $m=4$ we found $K(n, 4)=0$ for all $n>4$.

To sum up, within our limits of numerical accuracy and for the examples considered we have been able to confirm the asymptotic expansion (5-4) up to contributions going like $r^{-1 / 2}$; that is, for irreducible flat connections the coefficient in front of $\exp 2 \pi i r S_{\mathrm{CS}}(A)$ behaves like a constant and for

\begin{tabular}{|c|c|c|c|c|c|c|c|c|c|c|c|c|}
\hline$n$ & $m=1$ & 2 & 3 & 4 & 5 & 6 & 7 & 8 & 9 & 10 & 11 & 12 \\
\hline 0 & 0 & & & & & & & & & & & \\
\hline 1 & 1 & & & & & & & & & & & \\
\hline 2 & 2 & & & & & & & & & & & \\
\hline 3 & 3 & 0 & & & & & & & & & & \\
\hline 4 & 0 & & 0 & & & & & & & & & \\
\hline 5 & 1 & 2 & 2 & 0 & & & & & & & & \\
\hline 6 & 2 & & & & 2 & & & & & & & \\
\hline 7 & 3 & 0 & 3 & 0 & 3 & 0 & & & & & & \\
\hline 8 & 0 & & 1 & & 2 & & 0 & & & & & \\
\hline 9 & 1 & 2 & & 0 & 2 & & 0 & 0 & & & & \\
\hline 10 & 2 & & 2 & & & & 2 & & 2 & & & \\
\hline 11 & 3 & 0 & 0 & 0 & 1 & 0 & 2 & 2 & 1 & 0 & & \\
\hline 12 & 0 & & & & 2 & & 0 & & & & 0 & \\
\hline 13 & 1 & 2 & 1 & 0 & 1 & 2 & 2 & 1 & 1 & 0 & 2 & 0 \\
\hline 14 & 2 & & 3 & & 1 & & & & 2 & & 0 & \\
\hline 15 & 3 & 0 & & 0 & & & 3 & 0 & & & 1 & \\
\hline 16 & 0 & & 0 & & 0 & & 3 & & 2 & & 0 & \\
\hline 17 & 1 & 2 & 2 & 0 & 1 & 2 & 1 & 0 & 2 & 0 & 2 & 0 \\
\hline 18 & 2 & & & & 0 & & 1 & & & & 2 & \\
\hline 19 & 3 & 0 & 3 & 0 & 0 & 0 & 3 & 2 & 1 & 0 & 3 & 2 \\
\hline 20 & 0 & & 1 & & & & 1 & & 0 & & 0 & \\
\hline 21 & 1 & 2 & & 0 & 3 & & & 1 & & 2 & 2 & \\
\hline 22 & 2 & & 2 & & 0 & & 2 & & 0 & & & \\
\hline 23 & 3 & 0 & 0 & 0 & 3 & 0 & 2 & 0 & 1 & 2 & 3 & 0 \\
\hline 24 & 0 & & & & 3 & & 0 & & & & 1 & \\
\hline 25 & 1 & 2 & 1 & 0 & & 2 & 0 & 0 & 1 & & 3 & 0 \\
\hline 26 & 2 & & 3 & & 2 & & 2 & & 1 & & 0 & \\
\hline 27 & & & & & & & & 2 & & 2 & & \\
\hline 29 & & & & & & 2 & & & & 2 & & 0 \\
\hline 31 & & & & & & 0 & & & & 0 & & 2 \\
\hline 33 & & & & & & & & & & 0 & & \\
\hline 35 & & & & & & 0 & & & & & & 0 \\
\hline 37 & & & & & & & & & & & & 0 \\
\hline 41 & & & & & & & & & & & & 0 \\
\hline
\end{tabular}

TABLE 5. Values of $K$ for various manifolds $M_{8}(-n / m)$. 
reducible ones with $\operatorname{dim} H^{0}(A)=1$ like $r^{-1 / 2}$. In addition for some manifolds (including $n / m=7 / 1$, $16 / 1,22 / 1$ ) we have found peaks whose amplitudes indicate at least an $r^{-2}$ decay. To the best of our knowledge, so far such contributions to the semiclassical asymptotic expansion have no interpretation yet. The technique used to see the rate of decay was to modify Equation (5-5) by introducing the quantity

$$
\begin{aligned}
& \tilde{Z}_{l}\left(M_{8}(-n / m), t ; r_{\min }, r_{\max }, \alpha\right) \\
& =\left(r_{\max }-r_{\min }\right)^{-1} \sum_{r=r_{\min }}^{r=r_{\max }} r^{\alpha} Z_{l}\left(M_{8}(-n / m), r\right) e^{-2 \pi i t r},
\end{aligned}
$$

which satisfies

$$
\tilde{Z}_{l}\left(M_{8}(-n / m), t\right)=\tilde{Z}_{l}\left(M_{8}(-n / m, t ; 4,403,0) .\right.
$$

We then looked at the absolute value of the peaks for varying $r_{\min }, r_{\max }$, and $\alpha$.

Figure 16 gives an example for the case $n / m=$ $16 / 1$, for which $\exp i \psi=1$; see (5-8). The top row of diagrams, with $\alpha=0$, shows that the amplitudes for the irreducible flat connections (indicated by arrows: see Table 2) stay constant for varying $r_{\max }$ and $r_{\min }$. The middle row, with $\alpha=1 / 2$, shows that the amplitudes of the reducible flat connections (again indicated by arrows: see Table 1) behave like $r^{-1 / 2}$. The bottom row, with $\alpha=2$, indicates that the peak at 0.6104 (which is not the value for the Chern-Simons action of a flat connection) has an amplitude that decays at least like $r^{-2}$. In the two bottom rows the peaks corresponding to
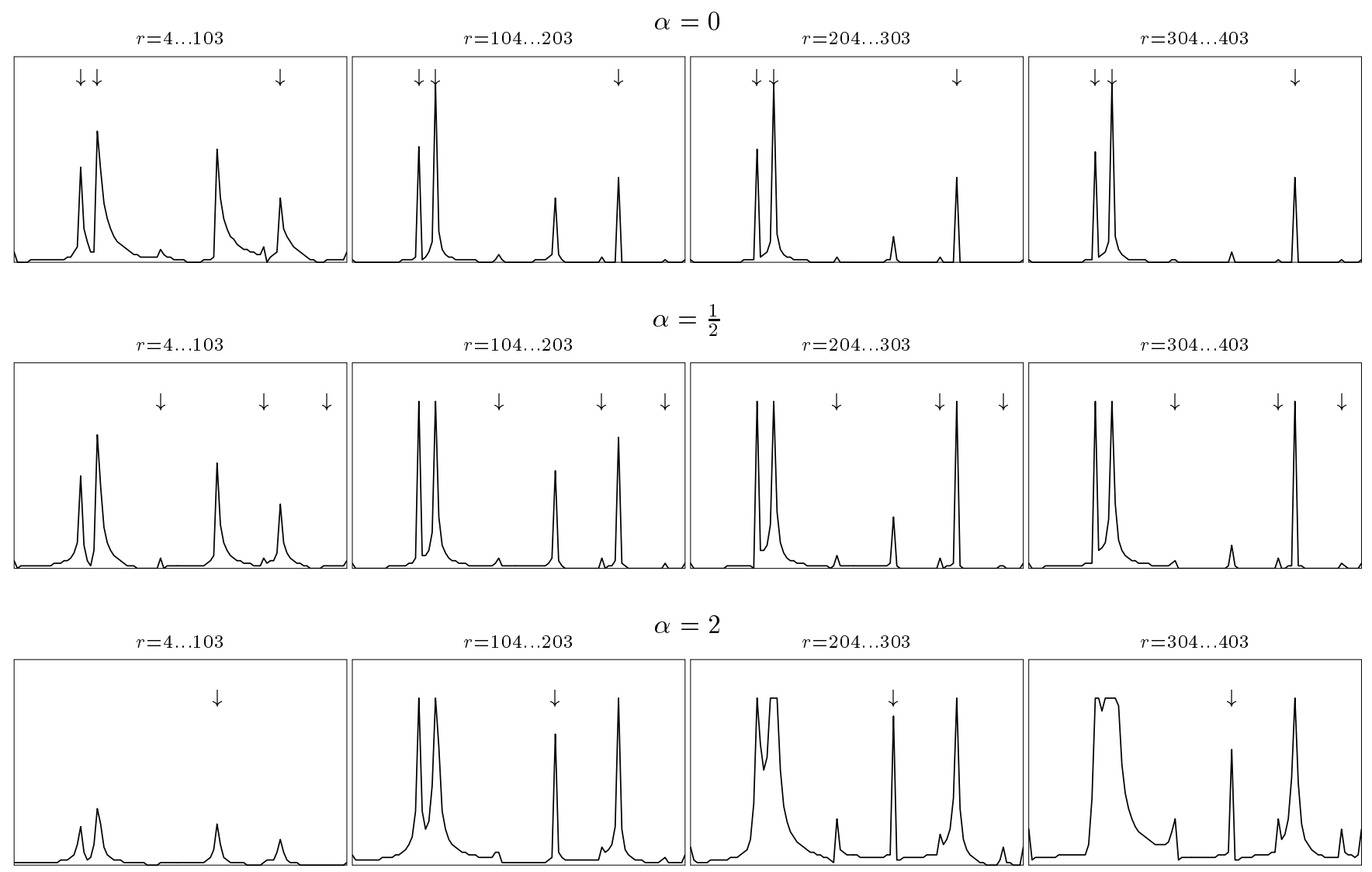

FIGURE 16. The absolute value $\left|\tilde{Z}_{l}\left(M_{8}(-16 / 1), t ; r_{\min }, r_{\max }\right)\right|$ of the Fourier transform of $r^{\alpha} Z_{l}\left(M_{8}(-16 / 1), r\right)$ for some $r$-regimes. 
irreducible flat connections have been cut off. Figure 16 also shows that for $Z_{l}\left(M_{8}(-16 / 1), r\right)$ the asymptotic regime sets in at $r \approx 100$.

\section{A. PROOF OF AN ORTHOGONALITY RELATION FOR THE CANONICAL GRAPH OF HANDLEBODIES}

In this appendix we outline the proof of Equation $(2-14)$. To this end we we cut the manifold $(2-13)$

$$
\left(M_{\Sigma}^{*} \backslash \mathcal{T}_{\mathcal{G}^{\Sigma^{*}}}\right) \bigcup_{\Sigma}\left(M_{\Sigma} \backslash \mathcal{T}_{\overline{\mathcal{G}} \Sigma}\right) \cong \Sigma \times I
$$

into simple pieces. We consider a hollow cylindric part which is the neighbourhood of the lines with colours $e=e_{i}, \bar{f}=\bar{f}_{i}, e^{\prime}=e_{i}^{\prime}, \bar{f}^{\prime}=\bar{f}_{i}^{\prime}$ for some $i$ in the range $1 \leq i \leq 3 g-3$. We apply some results from [Karowski and Schrader 1993] that are recalled in Section 2 and Appendix B: Lemma 2.10, which says how to introduce hollow tubes, and Lemma 2.9, which says how to cut full cylinders. Using in addition Equations (B-2) and (B-3) we have the following cutting rule:

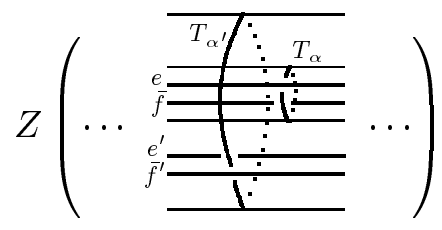

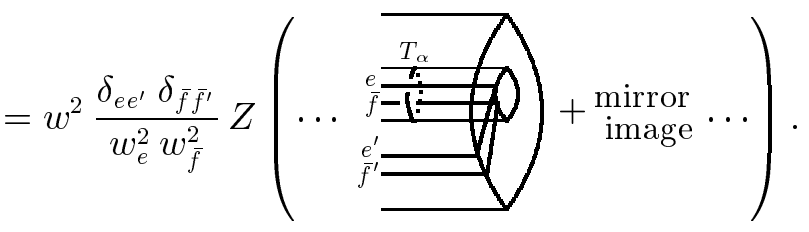

Applying this rule $3 g-3$ times, where $g>1$, the manifold (A-1) may be cut into $2 g-2$ pieces of the form

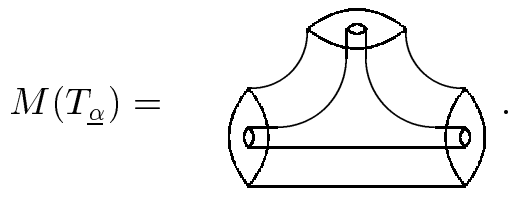

These manifolds are equipped with graphs

$$
|G|_{e_{1} e_{2} e_{3} \bar{f}_{1} \bar{f}_{2} \bar{f}_{2}},
$$

where the $e$-lines branch at a 3 -vertex and so do the $\bar{f}$-lines. In addition there are the meridians. Using again the cutting rules of [Karowski and Schrader 1993] one finds

$$
Z\left(M\left(T_{\underline{\alpha}}\right),|G|_{e_{1} e_{2} e_{3} \bar{f}_{1} \bar{f}_{2} \bar{f}_{2}}\right)=w^{-4} N_{e_{2} e_{3}}^{e_{1}} N_{\bar{f}_{2} \bar{f}_{3}}^{\bar{f}_{1}} .
$$

This concludes the proof of Equation (2-14).

\section{B. PROOF OF SOME EQUATIONS OF SECTION 3}

In this appendix we outline the proofs of some equations of Section 3. The following equations involving graphs are to be understood as equations of their invariants in the sense of Equation $(2-1)$. If there are graphs with open lines they have to be interpreted as parts of closed graphs. By $(2-2)-(2-5)$ and the Wigner-Eckart relation (2-6) we have the following completeness and Racah relations:

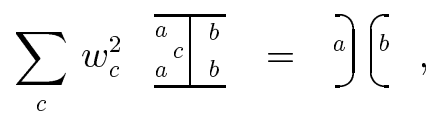

$$
\begin{aligned}
& \left.\frac{a}{\underline{b}} \underline{c}_{c}=\frac{q_{c}}{q_{a} q_{b}} \quad \vec{b}\right)_{c} .
\end{aligned}
$$

The Verlinde matrix (genus $g=1$ )

$$
S_{a b}=(-1)^{2 a+2 b} \frac{\sin (\pi / r)(2 a+1)(2 b+1)}{w \sin (\pi / r)}
$$

diagonalizes the fusion matrix; that is, it solves the eigenvalue equation

$$
\left(N^{d} S\right)_{a b}=\frac{S_{d b}}{S_{d 0}} S_{a b}
$$

and we have the well-known relations

$$
\frac{1}{w}\left[\begin{array}{l}
a \\
b
\end{array}=S_{a b}=S_{b a}=S_{a b}^{*}=\left(S^{-1}\right)_{a b} ;\right.
$$

see [Karowski and Schrader 1993], for example. 
In particular $S^{2}=1$, together with the WignerEckart relation (2-6), implies the following change of a part of a graph:

$$
\begin{aligned}
\sum_{b} w_{b}^{2}-\left[\begin{array}{l}
a \\
b
\end{array}\right. & \left.=\sum_{b} \frac{w_{b}^{2}}{w_{a}^{2}} a\right]\left(w_{b}{ }^{2}\right. \\
& \left.=\frac{w^{2}}{w_{a}^{2}} \sum_{b} S_{a b} S_{b 0} \quad a\right) \\
& \left.=w^{2} \delta_{a 0} a\right] ;
\end{aligned}
$$

compare [Karowski and Schrader 1993, (A.2)]. As a result, using the completeness relation (B-1), we get

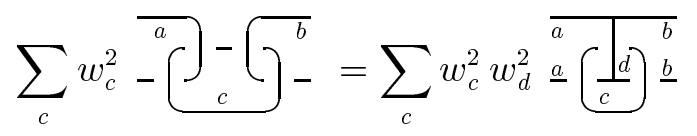

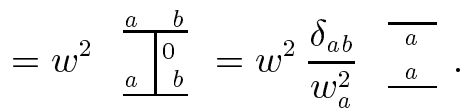

Also we have with Equation (B-1)

$$
\begin{aligned}
& \sum_{b} w_{b}^{2}-\bigodot_{b}^{a}=\sum_{b} w_{b}^{2} q_{b}^{2}-\complement_{b}^{a} \\
& =\sum_{b c} w_{b}^{2} w_{c}^{2} q_{b}^{2} \underline{\underline{a}} \underline{\underline{\rho}} \underline{\underline{b}} \\
& \left.=q_{a}^{-2} w \kappa^{a}\right]=w \kappa_{\underline{a}}^{-} P \text {, }
\end{aligned}
$$

and analogously to Equation (B-3)

$$
\sum_{c} w_{c}^{2} q_{c}^{2}-\underbrace{a}_{c}-\sqrt{b}=q_{a}^{-2} q_{b}^{-2} w \kappa{ }^{-} \sqrt{a}-
$$

where in both cases the sum over $c$ yields the constant

$$
w \kappa=\sum_{c} w_{c}^{4} q_{c}^{2}=w q^{-3 / 2} e^{-i \pi(1 / 4+r / 2)}
$$

In (B-4) and (B-5) we used Yang-Baxter relations and in (B-6) we used Gauss's reciprocity relation. These formulas for the special matrix $S_{a b}=S_{a b}^{0}$ are now used to derive Equations (3-19)-(3-23) for the general matrix $S_{a b}^{c}$ defined by Equation (3-12).

Proof of (3-18). The symmetry $S_{a b}^{c}=S_{b a}^{c}$ follows directly from the homotopy invariance of the invariants of graphs on $S^{2}$, and the second equation follows from

$$
\begin{aligned}
& \frac{w}{w_{a} w_{b}} S_{a b}^{c}={ }_{a}^{c} G^{b}=\overparen{L_{b}^{c}} \\
& =\overbrace{a}^{c} \overbrace{a}^{c}-1=q_{c} \frac{w}{w_{a} w_{b}}\left(S_{a b}^{c}\right)^{*} \text {. }
\end{aligned}
$$

Proof of (3-19). Equation (B-3) implies

$$
\begin{aligned}
\sum_{d} S_{a d}^{c} S_{d b}^{c *} & =\sum_{d} \frac{w_{a} w_{d}^{2} w_{b}}{w^{2}} \\
& =\sum_{d} \frac{w_{a} w_{d}^{2} w_{b}}{w^{2}}{ }_{a}^{c}-Z_{d}^{b}=\delta_{a b} N_{a a}^{c} .
\end{aligned}
$$

Proof of (3-21). Equation (B-5) implies

$$
\begin{aligned}
\sum_{d} S_{a d}^{c} q_{d}^{2} S_{d b}^{c *} & =\sum_{d} \frac{w_{a} w_{d}^{2} w_{b}}{w^{2}} q_{d}^{2}{ }^{a}{ }_{d}^{c} \frac{w_{a} w_{d}^{2} w_{b}}{w^{2}} q_{d}^{2}{ }_{d}^{c}-C_{d}^{b} \\
& =\kappa q_{a}^{-2} q_{b}^{-2} S_{a b}^{c *}
\end{aligned}
$$

and (3-21) follows with $S_{a b}^{c}=S_{b a}^{c}$.

Proof of (3-22) and (3-23). The eigenvalue equation of $N_{a b}^{c d}$ defined by $(3-17)$ reads

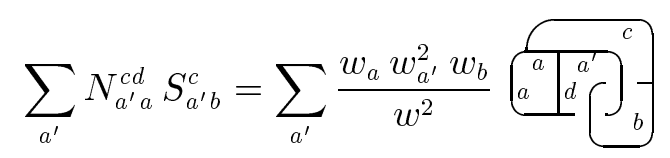

$$
\begin{aligned}
& =\frac{w_{a} w_{b}}{w^{2}} \overbrace{a}^{c}-\frac{S_{d b}}{S_{0 b}} S_{a b}^{c} \text {. }
\end{aligned}
$$


Proof of (3-17). Using again Equation (B-1) we obtain the following representation for the matrix $S_{a b}^{s}$ :

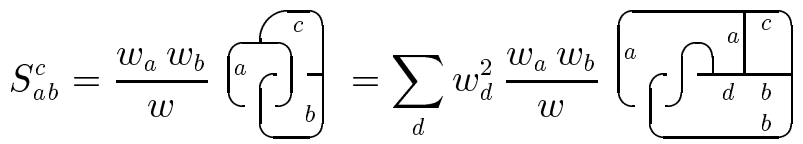

$$
\begin{aligned}
& =\frac{1}{w q_{a}^{2} q_{b}^{2}} \sum_{d} w_{d}^{2} q_{d}^{2} N_{a b}^{c d} .
\end{aligned}
$$

\section{CHERN-SIMONS INVARIANTS FOR FLAT CONNECTIONS}

The results of [Kirk and Klassen 1990] describe explicitly how to obtain all irreducible flat connections up to gauge equivalence of $M_{8}(-n / m)$ and give precise formulae for computing the corresponding Chern-Simons invariants. For the convenience of the reader, and since we derive some consequences from these formulae, we will briefly recall these results. The group $G$ of the figure-eight knot has the presentation

$$
\left\langle x, y:\left[x^{-1}, y\right] x=y\left[x^{-1}, y\right]\right\rangle,
$$

where $x$ represents a meridian and

$$
\lambda=\left[y, x^{-1}\right]\left[x, y^{-1}\right]
$$

the preferred longitudinal curve. Define curves in $\mathbb{C} \times \mathbb{R}$ by

$$
\left(e^{2 \pi i s}, u_{ \pm}(s)\right) \text { for } s \in\left[\frac{1}{6}, \frac{1}{3}\right]
$$

where

$u_{ \pm}(s)=\cos 4 \pi s-3 / 2 \pm \sqrt{\cos ^{2} 4 \pi s-\cos 4 \pi s-\frac{3}{4}}$.

Then the representations of $G$ given by

$$
\begin{aligned}
x & \mapsto\left(\begin{array}{cc}
e^{2 \pi i s} & e^{-2 \pi i s} \\
0 & e^{-2 \pi i s}
\end{array}\right), \\
y & \mapsto\left(\begin{array}{cc}
e^{2 \pi i s} & 0 \\
-e^{2 \pi i s} u_{ \pm}(s) & e^{-2 \pi i s}
\end{array}\right),
\end{aligned}
$$

for $s \in\left[\frac{1}{6}, \frac{1}{3}\right]$, are conjugate in $\mathrm{SL}(2, \mathbb{C})$ to irreducible $\mathrm{SU}(2)$ representations and correspond oneto-one to the conjugacy classes of irreducible $\mathrm{SU}(2)$ representations.

Actually, the above $\mathrm{SL}(2, \mathbb{C})$ representation is conjugate to a unique $\mathrm{SU}(2)$ representation $\rho_{s, \pm}$ having the following action:

$$
\begin{aligned}
x & \mapsto\left(\begin{array}{cc}
e^{2 \pi i s} & 0 \\
0 & e^{-2 \pi i s}
\end{array}\right), \\
\lambda & \mapsto\left(\begin{array}{cc}
L_{ \pm}(s) & 0 \\
0 & L_{ \pm}(s)^{-1}
\end{array}\right),
\end{aligned}
$$

with

$$
\begin{aligned}
L_{ \pm}(s)=-1+e^{-4 \pi i s}-2 e^{4 \pi i s} & +e^{8 \pi i s} \\
& +u_{ \pm}(s)\left(e^{-4 \pi i s}-e^{4 \pi i s}\right) .
\end{aligned}
$$

Now assume that $\rho_{s, \pm}$ is such that it projects to a representation of $\pi_{1}\left(M_{8}(n / m)\right)$. If $\gamma_{t}$, for $0 \leq t \leq$ 1 , is a path of $\operatorname{SL}(2, \mathbb{C})$ representations of $G$ going from the identity to $\rho_{s, \pm}$, and such that

$$
\begin{aligned}
& \gamma_{t}(x)=\left(\begin{array}{cc}
e^{2 \pi i \alpha(t)} & 0 \\
0 & e^{-2 \pi i \alpha(t)}
\end{array}\right), \\
& \gamma_{t}(\lambda)=\left(\begin{array}{cc}
e^{2 \pi i \beta(t)} & 0 \\
0 & e^{-2 \pi i \beta(t)}
\end{array}\right),
\end{aligned}
$$

it follows from [Kirk and Klassen 1990, Theorem 4.3] that the Chern-Simons invariant $S_{\mathrm{CS}}\left(\rho_{s, \pm}\right)$ of $M_{8}(n / m)$ corresponding to $\rho_{s, \pm}$ is equal to

$-2 \int_{0}^{1} \beta \alpha^{\prime} d t-\mu \cdot n \alpha^{2}(1)$

$$
-\nu \cdot m \beta^{2}(1)-2 \mu \cdot m \alpha(1) \beta(1) . \quad(\mathrm{C}-1)
$$

Here $\mu$ and $\nu$ are integers such that $\nu n-\mu m=1$. (This is formula $(*)$ of [Kirk and Klassen 1990, p. 361], taking account of a misprint).

In [Kirk and Klassen 1990, pp. 361, 362] a path of representations from id to $\rho_{1 / 6,+}=\rho_{1 / 6,-}$ is provided and the corresponding value of the integral $\int \beta \alpha^{\prime}$ up to this point is evaluated as $\frac{1}{12}$. The corresponding values of $\alpha$ and $\beta$ are $\frac{1}{6}$ and $\frac{1}{2}$. The representation $\rho_{s, \pm}$ of $G$, for $s \in\left[\frac{1}{6}, \frac{1}{3}\right]$, factors 
through a representation of $\pi_{1}\left(M_{8}(n / m)\right)$ if and only if $\rho_{s, \pm}\left(x^{n} \cdot \lambda^{m}\right)=\mathrm{id}$; that is, if and only if

$$
e^{-2 \pi i n s}=\left(L_{ \pm}(s)\right)^{m} \text {. }
$$

Define $\lambda_{ \pm}(s)$ by $L_{ \pm}(s)=e^{2 \pi i \lambda_{ \pm}(s)}$ such that $\lambda_{ \pm}$ is continuous and $\lambda_{ \pm}\left(\frac{1}{4}\right)=0$. One easily sees that $L_{ \pm}\left(\frac{1}{4}-s\right)=\overline{L_{ \pm}\left(\frac{1}{4}+s\right)}$, so that $\lambda_{ \pm}\left(\frac{1}{4}-s\right)=$ $-\lambda_{ \pm}\left(\frac{1}{4}+s\right)$. Further,

$$
\begin{aligned}
& L_{ \pm}\left(\frac{1}{4}+s\right)=-2 \sin ^{2} 4 \pi s \\
& \quad+\cos 4 \pi s \pm 2 i \sin 4 \pi s \sqrt{\left(\cos 4 \pi s+\frac{1}{2}\right)^{2}-1} .
\end{aligned}
$$

This implies that for $0 \leq s \leq \frac{1}{12}$ the function $\lambda_{+}\left(\frac{1}{4}+s\right)$ is strictly monotonically increasing from 0 to $\frac{1}{2}$ and $\lambda_{-}(s)=-\lambda_{+}(s)$.

It follows that $\rho_{s, \pm}$ factors through a representation of $M_{8}(n, m)$ if and only if

$$
n / m \cdot s \equiv \pm \lambda_{-}(s) \bmod (1 / m)
$$

The following facts are easy to check (we will always assume that $n, m$ are coprime).

- If $n$ is even, then $\frac{1}{4}+\tau$ is a solution if and only if $\frac{1}{4}-\tau$ is a solution.

- $\frac{1}{4}$ is a solution if and only if 4 divides $n$.

- If $s$ is a solution for $(n, m)$ then it is also a solution for $(-n, m)$ with the sign in $\rho_{s, \pm}$ changed.

Let $R(n / m, \pm)$ be the number of $s \in\left[\frac{1}{6}, \frac{1}{3}\right]$ such that $\rho_{s, \pm}$ projects to a representation of $\pi_{1} M_{8}(n / m)$. We will always assume that $m>0$. We have the following results:

- For $n / m \geq-2 \sqrt{5}$ (this being the maximum of $\lambda_{-}^{\prime}(s)$ for $\left.\frac{1}{6} \leq s \leq \frac{1}{3}\right), R(n / m,+)$ is the number of integers in $\left[-\frac{m}{2}-\frac{n}{3}, \frac{m}{2}-\frac{n}{6}\right]$.

- For $n / m \leq-12, R(n / m,+)$ is the number of integers in $\left[\frac{m}{2}-\frac{n}{6},-\frac{m}{2}-\frac{n}{3}\right]$.

- For $n / m \leq 2 \sqrt{5}, R(n / m,-)$ is the number of integers in $\left[-\frac{m}{2}-\frac{n}{6}, \frac{m}{2}-\frac{n}{3}\right]$.

- For $n / m \geq 12, R(n / m,-)$ is the number of integers in $\left[\frac{m}{2}-\frac{n}{3},-\frac{m}{2}-\frac{n}{6}\right]$.

To compute the Chern-Simons invariant $S_{\mathrm{CS}}\left(\rho_{s, \pm}\right)$ of $M_{8}(n / m)$ corresponding to the representation $\rho_{s, \pm}$ we employ $(\mathrm{C}-1)$. Using the path of [Kirk and Klassen 1990] from the trivial representation to $\rho_{1 / 6,+}=\rho_{1 / 6,-}$ and then the path $t \mapsto \rho_{t, \pm}$, with $t \in\left[\frac{1}{6}, s\right]$, we get for the Chern-Simons invariant of $\rho_{s,+}$ the value

$-2\left(\frac{1}{12}+\int_{\frac{1}{6}}^{s}\left(\lambda_{+}(t)+1\right) d t\right)$

$-\mu n s^{2}-\nu m\left(\lambda_{+}(s)+1\right)-2 \mu m s\left(\lambda_{+}(s)+1\right), \quad(\mathrm{C}-3)$

while the one corresponding to $\rho_{s,-}$ is

$$
\begin{aligned}
-2\left(\frac{1}{12}+\right. & \left.\int_{\frac{1}{6}}^{s} \lambda_{-}(t) d t\right) \\
& -\mu n s^{2}-\nu m \lambda_{-}(s)-2 \mu m s \lambda_{-}(s) . \quad(\mathrm{C}-4)
\end{aligned}
$$

(Since the curve $\beta$ of the path from id to $\rho_{1 / 6,+}$ ends with the value $\frac{1}{2}$, the continuation of $\beta$ from $\rho_{1 / 6,+}$ to $\rho_{s,+}$ is $\lambda_{+}(s)+1$, and not simply $\left.\lambda_{+}(s)\right)$.

Using these formulae and the fact that

$$
\lambda_{ \pm}\left(\frac{1}{4}+\tau\right)=-\lambda_{ \pm}\left(\frac{1}{4}-\tau\right),
$$

a straightforward calculation for even $n$ and for $s=\frac{1}{4}+\tau$ a solution of $(\mathrm{C}-2)$ yields $S_{\mathrm{CS}}\left(\rho_{1 / 4+\tau, \pm}\right)-$ $S_{\mathrm{CS}}\left(\rho_{1 / 4-\tau, \pm}\right) \equiv n \mu / 4 \bmod 1$, where as before $\mu$ is given by $\nu n-\mu m=1$. Therefore:

Proposition C.1. (a) Let $n, m$ be coprime with $n=$ $4 k+2$. Then for any irreducible representation $\rho_{1 / 4+\tau, \pm}$ of $\pi_{1}\left(M_{8}(n / m)\right)$ also $\rho_{1 / 4-\tau, \pm}$ is an irreducible representation and

$$
S_{\mathrm{CS}}\left(\rho_{1 / 4+\tau, \pm}\right)-S_{\mathrm{CS}}\left(\rho_{1 / 4-\tau, \pm}\right)=\frac{1}{2} \bmod 1 .
$$

(b) If $n=4 k$, we have the irreducible representations $\rho_{1 / 4, \pm}$ with Chern-Simons invariant

$$
\pm 1 / 5+l / 4 \quad \text { if } k \equiv l \bmod 4,
$$

and, for any irreducible representation $\rho_{1 / 4+\tau, \pm}$ of $\pi_{1}\left(M_{8}(n / m)\right)$, the representation $\rho_{1 / 4-\tau, \pm}$ is also irreducible and

$$
S_{\mathrm{CS}}\left(\rho_{1 / 4+\tau, \pm}\right)=S_{\mathrm{CS}}\left(\rho_{1 / 4-\tau, \pm}\right) .
$$

This result was inspired experimentally. First, our numerical calculations of $Z_{l}\left(M_{8}(-n / 1), r\right)$ for $n \equiv$ $2 \bmod 4$ suggested that these numbers are 0 for all 
odd $r$. Then we proved this analytically (see Appendix D). This implies that the Fourier transform $\tilde{Z}_{l}\left(M_{8}(-n / 1), t\right)$ is periodic with period $\frac{1}{2}$. Assuming the correctness of the semiclassical approximation formula (5-4) a statement like Proposition C.1 would have to hold.

The statement that $Z_{l}\left(M_{8}(-n, 1), r\right)=0$ for $n \equiv 2 \bmod 4$ and $r$ odd may be generalized as follows:

Proposition C.2. Let $M_{K}(n / m)$ be the three-manifold obtained by $(n / m)$ Dehn surgery on the knot $K \subset S^{3}$. If $n \equiv 2 \bmod 4$ (and $m, n$ coprime) then $Z_{l}\left(M_{K}(n / m), r\right)=0$ for every odd $r$.

When $K$ is the figure-eight knot, this result turns into an if and only if, with a proof that uses different arguments; see Appendix D.

Proof. By [Kirby and Melvin 1991, Corollary 8.9] it suffices to show that $Z_{l}\left(M_{K}(4 k+2 / 2 l+1), 3\right)=0$, which in turn holds if and only if one can embed a closed surface of odd Euler characteristic in $M_{K}(4 k+2 / 2 l+1)$ [Kirby and Melvin 1991, Theorem 6.3]. If $D^{2} \times S^{1}$ is the solid torus glued into the exterior of a tubular neighborhood of $K$ to obtain $M_{K}(4 k+2 / 2 l+1)$, then a Seifert surface of $K$ will meet the boundary torus $S^{1} \times S^{1}$ of $D^{2} \times S^{1}$ in a simple closed curve homologous to $-p\left(S^{1} \times\{*\}\right)+(4 k+2)\left(\{*\} \times S^{1}\right)$, where $p$ is an integer such that $p(2 l+1) \equiv-1 \bmod (4 k+2)$. Any such curve bounds a nonorientable surface of genus $2 k+1$ in $D^{2} \times S^{1}$. The union of these two surfaces is then a closed surface of odd Euler characteristic.

With regard to reducible representations, note that the first integral homology group of $M_{8}(n / m)$ is isomorphic to $\mathbb{Z} / n \mathbb{Z}$. Therefore, $\pi_{1}\left(M_{8}(n / m)\right)$ has nontrivial reducible $\mathrm{SU}(2)$ representations if $|n|>1$. They correspond to the representations of $\pi_{1}(L(n, m))$, where $L(n, m)$ is the $(n, m)$-lens space. The proof of [Kirk and Klassen 1990, Theorem 5.1] can be applied to calculate the set of Chern-Simons invariants of reducible representations of $M_{K}(n / m)$ for any knot $K$ in $S^{3}$. Therefore, the set of Chern-Simons invariants of $M_{K}(n / m)$ corresponding to reducible flat connections $A$ (for these, as noted in Section $5, \operatorname{dim} H^{0}(A)=1$ unless $A$ is trivial) is given by

$$
\left\{-\frac{t^{2} p}{n} \mid t=0,1, \ldots,\left\lfloor\frac{n}{2}\right\rfloor\right\}
$$

where as before $p$ is an integer such that $p \cdot m \equiv-1$ $\bmod n$.

\section{VANISHING INVARIANTS}

Here we give a necessary and sufficient condition for $Z_{l}\left(M_{8}(-n / m), 3\right)$ to be zero. By a result from [Kirby and Melvin 1991], $Z_{l}\left(M_{8}(-n / m), r\right)$ vanishes for $r$ odd whenever

$$
Z_{l}\left(M_{8}(-n / m), 3\right)=0 .
$$

For $r=3$ we have

$$
A=\left(\begin{array}{ll}
1 & 0 \\
0 & i
\end{array}\right), \quad S=\frac{1}{\sqrt{2}}\left(\begin{array}{cc}
1 & -1 \\
-1 & -1
\end{array}\right)
$$

and

$$
\left|\begin{array}{lll}
e & e & 0 \\
e & e & 0
\end{array}\right|=(-1)^{2 e}=w S_{0 e} \quad \text { for } e=0, \frac{1}{2} .
$$

We consider the case $\left|\frac{n}{m}\right|>1$ first.

Since $S^{2}=1$ we have by (4-9) and (4-13)

$$
\begin{aligned}
& Z_{l}\left(M_{8}(-n / m), 3\right) \\
& =e^{i \psi} w^{-1} \sum_{e}(-1)^{2 e}\left\langle e\left|A^{m_{t}} S \cdots S A^{m_{1}} S\right| 0\right\rangle \\
& =e^{i \psi}\left\langle 0\left|S A^{m_{t}} S \cdots S A^{m_{1}} S\right| 0\right\rangle
\end{aligned}
$$

where $\left(m_{t}, \ldots, m_{1}\right)$ is the continued fraction expansion of $n / m$ (compare Section 4 ). Consider

$$
X\left(m_{t}, \ldots, m_{1}\right)=S A^{m_{t}} S \cdots S A^{m_{1}} S
$$

as a word in $S$ and $A$. We call words of the form $S A^{\alpha} S A^{k}$ for $\alpha$ even and those of the form

$$
S A^{\alpha} S A^{\beta_{r}} \cdots S A^{\beta_{1}} S A^{\gamma} S A^{k}
$$

for $\alpha, \gamma$ odd, $\beta_{j}$ even, and $0 \leq r$ initial factors if $k \neq 0$ and final factors if $k=0$. A (final or initial) factor of the form $S A^{4 k} S A^{k}$ or of the form 
$S A^{\alpha} S A^{\beta r} \cdots S A^{\beta_{1}} S A^{\gamma} S A^{k}$ with $\gamma \equiv \alpha \bmod 4$ is called even, all other factors are called odd. One can write $X\left(m_{t}, \cdots m_{1}\right)$ uniquely as

$$
\begin{aligned}
& I_{p} \cdots I_{1} \cdot F \quad \text { or } \\
& I_{p} \cdots I_{1} \cdot S A^{\gamma} S \quad \text { with } \gamma \text { odd, or } \\
& S A^{\alpha} S A^{\beta_{r}} \cdots S A^{\beta_{1}} S \quad \text { with } \alpha \text { odd, } \beta_{j} \text { even, }
\end{aligned}
$$

where the $I_{j}$ are initial factors and $F$ is a final factor.

Lemma D.1. Let $|n / m|>1$. We have

$$
Z_{l}\left(M_{8}(-n / m), 3\right)=e^{i \psi}\left\langle 0\left|X\left(m_{t}, \cdots m_{1}\right)\right| 0\right\rangle=0
$$

if and only if $X\left(m_{t}, \ldots, m_{1}\right)=I_{p} \cdots I_{1} \cdot F$, where the $I_{j}$ are initial factors, $F$ is a final factor and the number of odd factors is odd.

Proof. Use the fact that $A^{4}=1$ and $\left(S A^{2}\right)^{4}=-1$ to check that even factors are diagonal matrices with both diagonal entries different from zero, while odd factors are off-diagonal matrices with both off diagonal entries different from zero (since their determinant is nonzero). The lemma follows from the equations

$$
\begin{aligned}
S A S & =\frac{1}{2}\left(\begin{array}{rr}
1-i & -1-i \\
-1-i & 1-i
\end{array}\right), \\
S A^{3} S & =\frac{1}{2}\left(\begin{array}{rr}
1+i & -1+i \\
-1+i & 1+i
\end{array}\right),
\end{aligned}
$$

and

$$
\begin{array}{rl}
S A^{\alpha} S A^{\beta_{r}} \cdots S A^{\beta_{1}} & S \\
& =S A^{\alpha} S A^{\beta_{r}} \cdots S A^{\beta_{1}} S A^{\alpha} S S A^{4-\alpha} \\
& =D S A^{4-\alpha}
\end{array}
$$

with $D$ diagonal. For all odd $\alpha$ all entries of $S A^{4-\alpha}$ are nonzero.

When comparing Lemma D.1 with Proposition C.2 it turns out that in the case of Dehn surgery on the figure-eight knot Proposition C.2 gives exactly the surgery coefficients for which $Z_{l}\left(M_{8}(-n / m), 3\right)$ vanishes. With the preceding notation:
Lemma D.2. Let $\left(m_{t}, \ldots, m_{1}\right)$ be the continued fraction expansion of $n / m$, with $|n / m|>1$, and let $X\left(m_{t}, \ldots, m_{1}\right)$ be

(i) $I_{p} \cdots I_{1} \cdot F$ or

(ii) $I_{p} \cdots I_{1} \cdot S A^{\gamma} S \quad$ with $\gamma$ odd, or

(iii) $S A^{\alpha} S A^{\beta_{r}} \cdots S A^{\beta_{1}} S$ with $\alpha$ odd, $\beta_{j}$ even.

Then $n / m=4 k /(2 l+1)$ if and only if case (i) occurs with an even number of odd factors, and $n / m=(4 k+2) /(2 l+1)$ if and only if case (i) occurs with an odd number of odd factors.

Proof. We use induction on the length $t$ of the continued fraction expansion. Write

$$
\begin{aligned}
\frac{n}{m} & =m_{t}-\frac{1}{m_{t-1}-\frac{1}{\cdots-\frac{1}{m_{1}}}} \\
& =m_{t}-\frac{1}{m_{t-1}-\frac{1}{\cdots-\frac{1}{m_{s+1}-\frac{1}{q}}}},
\end{aligned}
$$

so that $\left(m_{s}, \ldots, m_{1}\right)$ is the continued fraction expansion of $q$. Notice that the denominator and numerator of a number described by a continued fraction expansion both change additively by multiples of 4 if the coefficients are changed additively by multiples of 4 . Then check that if $S A^{\alpha} S A^{k}$ or $S A^{\alpha} S A^{\beta_{r}} \ldots S A^{\beta_{1}} S A^{\gamma} S$ is an even or odd factor and $q=2 a /(2 l+1)$, then

$$
\alpha-\frac{1}{i-\frac{1}{q}} \quad \text { or } \quad \alpha-\frac{1}{\beta_{r}-\frac{1}{\cdots-\frac{1}{\gamma-\frac{1}{q}}}}
$$

is of the form $2 a^{\prime} /\left(2 l^{\prime}+1\right)$ with $2 a^{\prime} \equiv 2 a \bmod 4$ and $2 a^{\prime} \equiv 2 a+2 \bmod 4$, respectively. Analogously a final factor represents a fraction of the form $2 b /(2 l+1)$ with $b$ even or odd according to whether the final factor is even or odd. In case (ii) 
the induction starts with $q=\alpha=o d d$. The induction step of adding as above the coefficients of an initial factor to the coefficients of $q$ will change a fraction $q$ of the form odd/something into a $q^{\prime}$ of the same form.

Finally a direct calculation shows that the fraction represented by $(\alpha, 2,2,2, \ldots)$ equals $(\alpha(k+1)-$ $k) /(k+1)$, where $k$ is the number of 2's. If $\alpha$ is odd, then so is $\alpha(k+1)-k$. This takes care of case (iii), concluding the proof of the lemma.

Completely analogous results hold for $|n / m|<1$. Then

$$
\begin{aligned}
& Z_{l}\left(M_{8}(-n / m), 3\right) \\
& =e^{i \psi}\left\langle 0\left|A^{m_{t}} S A^{m_{t-1}} \cdots S A^{m_{1}} S\right| 0\right\rangle,
\end{aligned}
$$

where now $\left(m_{t}, \ldots, m_{1}\right)$ is the continued fraction expansion for $\left|\frac{m}{n}\right|$. Due to the above form of $A$ when $r=3$, we have

$$
\begin{aligned}
\left\langle 0\left|A^{m_{t}} S A^{m_{t-1}} \cdots S A^{m_{1}} S\right| 0\right\rangle & \\
& =\left\langle 0\left|S A^{m_{t-1}} \cdots S A^{m_{1}} S\right| 0\right\rangle .
\end{aligned}
$$

Therefore $\left(m_{t-1}, \ldots, m_{1}\right)$ is the continued fraction expansion of

$$
\frac{|n|}{m_{t}|n|-|m|}
$$

with $|n|>m_{t}|n|-|m|>0$. Thus $n / m$ is of the form $4 k /$ odd, $(4 k+2) /$ odd or odd/something if and only if $|n| /\left(m_{t}|n|-|m|\right)$ is.

\section{FINAL NOTE}

After the submission of this paper, R. J. Harrington's Ph.D. thesis [Harrington 1996] became available. It contains results overlapping to a certain degree with ours, although his focus is different. He compares directly the predicted and exact values of $Z(M, k)$ for large $k$ for $M$ obtained by $n / 1$ surgery from the figure-eight knot for some $n$. For these $n$ he calculates also the spectral flow and Reidemeister torsion that appear in the asymptotic formula for $Z(M, k)$. Reidemeister torsions for flat connections of manifolds obtained by $n / m$-surgery on the figure-eight knot were also computed by
Ch. Haase in his Diplomarbeit [Haase 1997]. In contrast our strategy was to determine the ChernSimons invariants from $Z(M, k)$. The method we use is Fourier analysis as suggested by the asymptotic formula. The same technique also allows the determination of the Reidemeister torsions and the spectral flows. So far agreement for the Reidemeister torsion is again excellent.

\section{REFERENCES}

[Atiyah 1990] M. Atiyah, "On framings of 3-manifolds", Topology 29:1 (1990), 1-7.

[Beliakova and Durhuus 1995] A. Beliakova and B. Durhuus, "Topological quantum field theory and invariants of graphs for quantum groups", Comm. Math. Phys. 167:2 (1995), 395-429.

[Durhuus 1993] B. Durhuus, "A discrete approach to topological quantum field theories", J. Geom. Phys. 11:1-4 (1993), 155-180.

[Durhuus et al. 1993] B. Durhuus, H. P. Jakobsen, and R. Nest, "Topological quantum field theories from generalized 6 $j$-symbols", Rev. Math. Phys. 5:1 (1993), 1-67.

[Freed and Gompf 1991] D. S. Freed and R. E. Gompf, "Computer calculation of Witten's 3-manifold invariant", Comm. Math. Phys. 141:1 (1991), 79-117.

[Fröhlich and King 1989] J. Fröhlich and C. King, "The Chern-Simons theory and knot polynomials", Comm. Math. Phys. 126:1 (1989), 167-199.

[Gepner and Witten 1986] D. Gepner and E. Witten, "String theory on group manifolds", Nuclear Phys. B 278:3 (1986), 493-549.

[Haase 1997] C. Haase, Reidemeister-Torsion und Chirurgien an der Achterschlinge, Diplomarbeit, Freie Universität Berlin, February 1997.

[Harrington 1996] R. J. Harrington, Witten's SU(2) invariant for a family of hyperbolic 3-manifolds, Ph.D. thesis, University of Texas, Austin, TX, December 1996.

[Heegaard 1916] P. Heegaard, "Sur l'Analysis situs", Bull. Soc. Math. France 44 (1916), 161-242.

[Jeffrey 1992] L. C. Jeffrey, "Chern-Simons-Witten invariants of lens spaces and torus bundles, and the 
semiclassical approximation", Comm. Math. Phys. 147:3 (1992), 563-604.

[Jones 1985] V. F. R. Jones, "A polynomial invariant for knots via von Neumann algebras", Bull. Amer. Math. Soc. (N.S.) 12:1 (1985), 103-111.

[Jones 1987] V. F. R. Jones, "Hecke algebra representations of braid groups and link polynomials", Ann. of Math. (2) 126:2 (1987), 335-388.

[Karowski 1992] M. Karowski, "Topological quantum field theories in terms of coloured graphs associated to quantum groups", pp. 118 in Group Theoretical Methods in Physics (Salamanca, 1992), vol. 2, edited by M. A. del Olmo et al., Ciemat, Madrid, 1992.

[Karowski and Schrader 1993] M. Karowski and R. Schrader, "A combinatorial approach to topological quantum field theories and invariants of graphs", Comm. Math. Phys. 151:2 (1993), 355-402.

[Karowski et al. 1992] M. Karowski, W. Müller, and R. Schrader, "State sum invariants of compact 3manifolds with boundary and $6 j$-symbols", J. Phys. A 25:18 (1992), 4847-4860.

[Kirby and Melvin 1991] R. Kirby and P. Melvin, "The 3 -manifold invariants of Witten and ReshetikhinTuraev for $\operatorname{sl}(2, \mathbb{C})$ ", Invent. Math. 105:3 (1991), 473-545.

[Kirillov and Reshetikhin 1989] A. N. Kirillov and N. Y. Reshetikhin, "Representation of the Algebra $U_{q}(\operatorname{sl}(2))$, q-orthogonal Polynomials and invariants of links", in New developments in the theory of knots, edited by T. Kohno, Advances series in Mathematical Physics 11, World Scientific, Singapore, 1989.

[Kirk and Klassen 1990] P. A. Kirk and E. P. Klassen, "Chern-Simons invariants of 3-manifolds and representation spaces of knot groups", Math. Ann. 287:2 (1990), 343-367.

[Kohno 1992] T. Kohno, "Topological invariants for 3 -manifolds using representations of mapping class groups, I", Topology 31:2 (1992), 203-230.

[Kohno 1994] T. Kohno, "Topological invariants for 3-manifolds using representations of mapping class groups, II: Estimating tunnel number of knots", pp. 193-217 in Mathematical aspects of conformal and topological field theories and quantum groups (South Hadley, MA, 1992), Contemp. Math. 175, Amer. Math. Soc., Providence, RI, 1994.
[Lawrence 1995] R. J. Lawrence, "Asymptotic expansions of Witten-Reshetikhin-Turaev invariants for some simple 3-manifolds", J. Math. Phys. 36:11 (1995), 6106-6129.

[Moore and Seiberg 1989] G. Moore and N. Seiberg, "Classical and quantum conformal field theory", Comm. Math. Phys. 123:2 (1989), 177-254.

[Murakami 1995] H. Murakami, "Quantum SO(3)invariants dominate the $\mathrm{SU}(2)$-invariant of Casson and Walker", Math. Proc. Cambridge Philos. Soc. 117:2 (1995), 237-249.

[Ohtsuki 1995] T. Ohtsuki, "A polynomial invariant of integral homology 3-spheres", Math. Proc. Cambridge Philos. Soc. 117:1 (1995), 83-112.

[Ohtsuki 1996] T. Ohtsuki, "A polynomial invariant of rational homology 3-spheres", Invent. Math. 123:2 (1996), 241-257.

[Osterwalder and Schrader 1973] K. Osterwalder and R. Schrader, "Axioms for Euclidean Green's functions", Comm. Math. Phys. 31 (1973), 83-112.

[Osterwalder and Schrader 1975] K. Osterwalder and R. Schrader, "Axioms for Euclidean Green's functions. II", Comm. Math. Phys. 42 (1975), 281-305.

[Reshetikhin and Turaev 1990] N. Y. Reshetikhin and V. G. Turaev, "Ribbon graphs and their invariants derived from quantum groups", Comm. Math. Phys. 127:1 (1990), 1-26.

[Reshetikhin and Turaev 1991] N. Reshetikhin and V. G. Turaev, "Invariants of 3-manifolds via link polynomials and quantum groups", Invent. Math. 103:3 (1991), 547-597.

[Rozansky 1995] L. Rozansky, "A large $k$ asymptotics of Witten's invariant of Seifert manifolds", Comm. Math. Phys. 171:2 (1995), 279-322.

[Rozansky 1997] L. Rozansky, "The trivial connection contribution to Witten's invariant and finite type invariants of rational homology spheres", Comm. Math. Phys. 183:1 (1997), 23-54.

[Spanier 1966] E. H. Spanier, Algebraic topology, McGraw-Hill, New York, 1966.

[Turaev 1991a] V. Turaev, "Quantum invariants of 3manifolds and a glimpse of shadow topology", C. R. Acad. Sci. Paris Sér. I Math. 313:6 (1991), 395-398. 
[Turaev 1991b] V. G. Turaev, "State sums in lowdimensional topology", pp. 689-698 in Proc. International Congress of Mathematicians (Kyoto, 1990), vol. 1, 1991.

[Turaev 1991c] V. G. Turaev, "Topology of shadows", preprint, 1991.

[Turaev 1992] V. G. Turaev, "Quantum invariants of 3-manifolds I and II", preprint 1992/015, Université de Strasbourg, 1992.

[Turaev 1993] V. Turaev, "Quantum invariants of links and 3-valent graphs in 3-manifolds", Inst. Hautes Études Sci. Publ. Math. 77 (1993), 121-171.
[Turaev and Viro 1992] V. G. Turaev and O. Y. Viro, "State sum invariants of 3-manifolds and quantum 6j-symbols", Topology 31:4 (1992), 865-902.

[Wajnryb 1983] B. Wajnryb, "A simple presentation for the mapping class group of an orientable surface", Israel J. Math. 45:2-3 (1983), 157-174.

[Walker 1991] K. Walker, “On Witten's three-manifold invariants", preprint, 1991.

[Wenzl 1993] H. Wenzl, "Braids and invariants of 3manifolds", Invent. Math. 114:2 (1993), 235-275.

[Witten 1989] E. Witten, "Quantum field theory and the Jones polynomial", Comm. Math. Phys. 121:3 (1989), 351-399.

Michael Karowski, Institut für Theoretische Physik, Freie Universität Berlin, WE2, Arnimallee 14, D-14195 Berlin, Germany (karowski@physik.fu-berlin.de)

Robert Schrader, Institut für Theoretische Physik, Freie Universität Berlin, WE2, Arnimallee 14, D-14195 Berlin, Germany (schrader@physik.fu-berlin.de)

Elmar Vogt, Institut für Mathematik, Freie Universität Berlin, WE2, Arnimallee 14, D-14195 Berlin, Germany (vogt@math.fu-berlin.de)

Received August 20, 1996; accepted in revised form May 7, 1997. 